University of Louisville

ThinkIR: The University of Louisville's Institutional Repository

Electronic Theses and Dissertations

$12-2017$

\title{
Testing and modeling of the thixotropic behavior of cementitious materials.
}

Bashir Hasanzadeh

University of Louisville

Follow this and additional works at: https://ir.library.louisville.edu/etd

Part of the Civil Engineering Commons

\section{Recommended Citation}

Hasanzadeh, Bashir, "Testing and modeling of the thixotropic behavior of cementitious materials." (2017). Electronic Theses and Dissertations. Paper 2868.

https://doi.org/10.18297/etd/2868

This Doctoral Dissertation is brought to you for free and open access by ThinkIR: The University of Louisville's Institutional Repository. It has been accepted for inclusion in Electronic Theses and Dissertations by an authorized administrator of ThinkIR: The University of Louisville's Institutional Repository. This title appears here courtesy of the author, who has retained all other copyrights. For more information, please contact thinkir@louisville.edu. 


\title{
TESTING AND MODELING OF THE THIXOTROPIC BEHAVIOR OF CEMENTITIOUS MATERIALS
}

\author{
By \\ Bashir Hasanzadeh \\ B.S., Ferdowsi University of Mashhad, Iran, 2010 \\ M.S., University of Birmingham, UK, 2011

\begin{abstract}
A Dissertation
Submitted to the Faculty of

In Partial Fulfillment of the Requirements

for the Degree of

Doctor of Philosophy

in Civil Engineering

Department of Civil and Environmental Engineering

University of Louisville

Louisville, Kentucky
\end{abstract} \\ J.B. Speed School of Engineering of University of Louisville
}

December 2017 
Copyright 2017 by Bashir Hasanzadeh

All rights reserved 



\title{
TESTING AND MODELING OF THE THIXOTROPIC BEHAVIOR OF CEMENTITIOUS MATERIALS
}

\author{
By \\ Bashir Hasanzadeh \\ B.S., Ferdowsi University of Mashhad, Iran, 2010 \\ M.S., University of Birmingham, UK, 2011
}

A Dissertation Approved on

November 15, 2017

By the following dissertation committee

Dr. Zhihui Sun (Dissertation Director)

Dr. Thomas Rockaway

Dr. Young Hoon Kim

Dr. Xiao-An Fu 


\section{ACKNOWLEDGEMENTS}

I would like to express my deepest gratitude to my advisor, Professor Zhihui Sun, who gave me the opportunity to work on this project under her supervision. Without her support, guidance, and encouragement I could not have made it to this stage of my Ph.D. study. She guided me throughout the whole program with great patience, inspiring discussions, excellent insights, and supportive encouragement.

I also want to thank Professor Thomas D. Rockaway, Professor Young Hoon Kim, and Professor Xiao-An Fu for serving on my dissertation committee.

The staff and students of the Department of Civil and Environmental Engineering deserve my special gratitude for creating a friendly and productive work environment. I especially would like to thank Mr. Mahyar Ramezani and Mr. Bernie Miles.

The financial supports from the National Science Foundation (CMMI-1265983, CMMI-1413031), Kentucky Science and Engineering Foundation (KSEF-3242-RDE-018) and the Department of Civil and Environmental Engineering at the University of Louisville, are highly appreciated. I also want to thank Cemex Technical Center for cement chemical composition analysis.

Finally, I would heartily like to thank my family who have supported me throughout my life and without them I could not finish this work. 


\section{ABSTRACT \\ TESTING AND MODELING OF THE THIXOTROPIC BEHAVIOR OF CEMENTITIOUS MATERIALS}

\section{Bashir Hasanzadeh}

November 8, 2017

During construction, concrete goes under various operations. It is normally transported to the job site, be placed and consolidated in the formwork, etc. The easiness and quality of these operations are very much related to the intrinsic rheological properties of concrete. Therefore, a good understanding of rheological behavior of concrete under various conditions are needed.

Thixotropy is an important part of rheology. The thixotropic behaviors of concrete can be good representatives of its flow behaviors on the job site. For example, the breakdown aspect of thixotropy can help to explain how its flow behavior can impact concrete pumping and its buildup aspect can help to estimate the lateral pressure on formwork and concrete resistance to segregation. Thus, good understanding of thixotropic behaviors of concrete can help to understand and predict flow behaviors of concrete in practice.

Researchers have realized the importance of thixotropic behavior of concrete, however, there is no agreement on the measuring protocol of this parameter. This leads to the goal of this research, which is to propose a comprehensive series of protocols that can make 
quantitative measurements on both breakdown and buildup aspects of thixotropic behaviors of concrete. While measuring breakdown and buildup separately, the correlations between these two aspects have also been investigated.

On top of the developed measuring protocol, a new analytical model on thixotropic behavior of cement paste is also proposed. With the proposed model, the influences of raw material characterizations and ambient condition on internal structural development in cement paste can be systematically studied.

To simulate the real on-site conditions, parameters such as pressure and temperature which influence rheological properties are also considered. 


\section{TABLE OF CONTENTS}

ACKNOWLEDGEMENTS .....................................................................................................III

ABSTRACT ................................................................................................................. IV

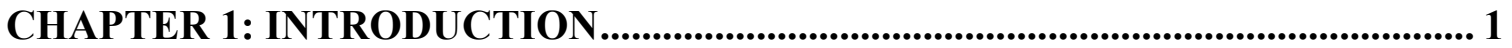

1.1. Fresh ANd RheOlogical Properties of CONCRETE ............................................ 1

1.2. RESEARCH SIGNIFICANT AND OBJECTIVES ..................................................... 3

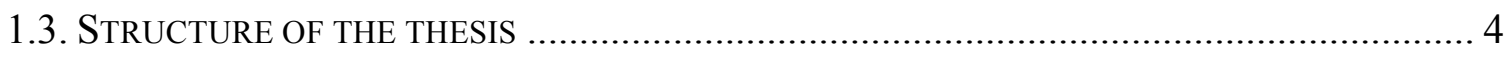

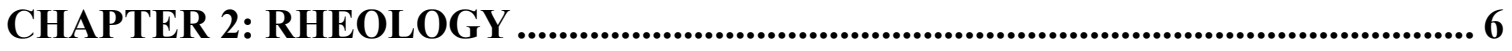

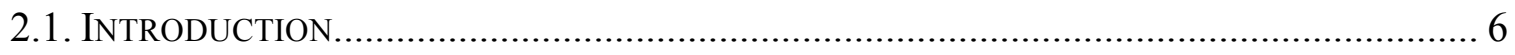

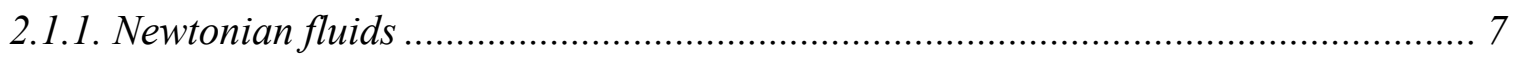

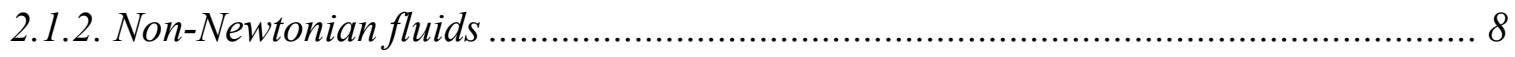

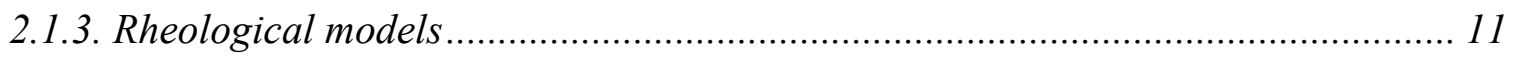

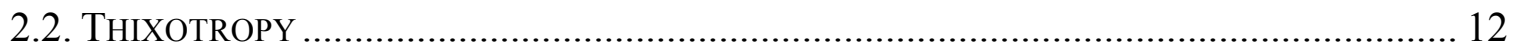

2.2.1. Current methods of measuring thixotropy of cementitious materials................... 13

2.2.2. Factors influencing rheology of cementitious materials ................................... 16

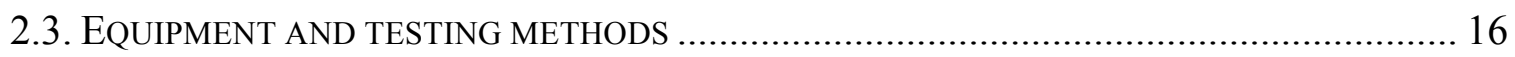

CHAPTER 3: FRESH PROPERTIES OF CEMENT PASTES WITH DIFFERENT DIATOMACEOUS EARTH CONTENTS ............................................................. 20

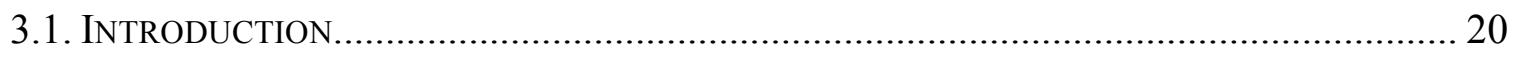

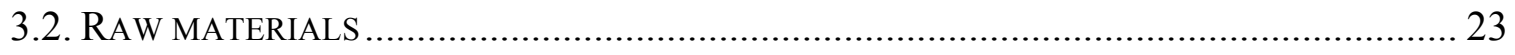


3.3. MIXING PROCEDURES AND TESTING MATRIX

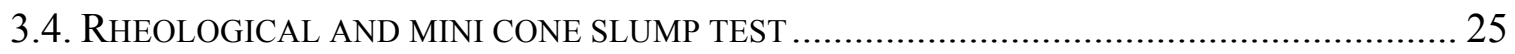

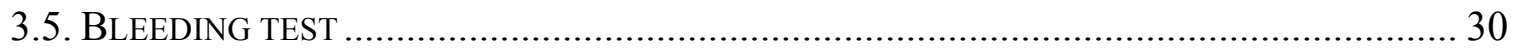

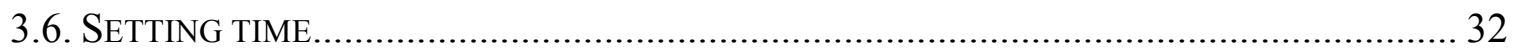

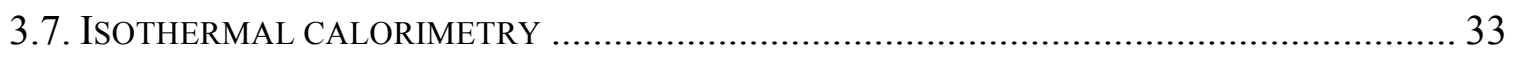

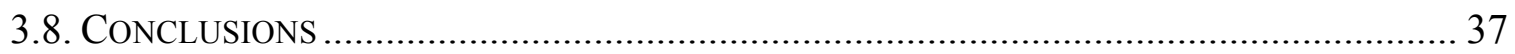

CHAPTER 4: THIXOTROPIC PROTOCOLS......................................................... 39

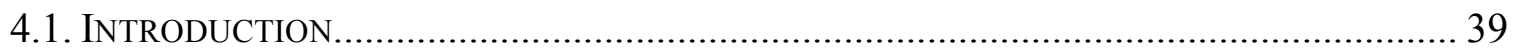

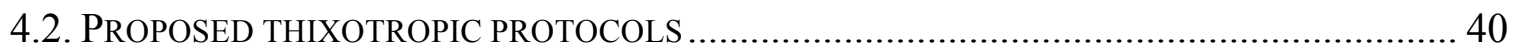

4.2.1. Protocol 1 (easiness of breaking down to steady state) ..................................... 40

4.2.2. Protocol 2 (easiness of starting the flow) ........................................................... 41

4.2.3. Protocol 3 (measuring buildup ratio by monitoring shear stress buildup) ........... 42

4.2.4. Protocol 4 (measuring buildup ratio by monitoring static yield stress)................ 43

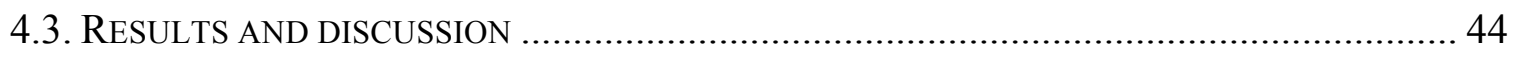

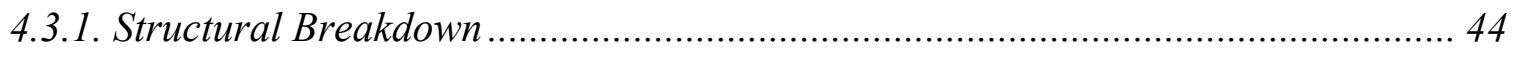

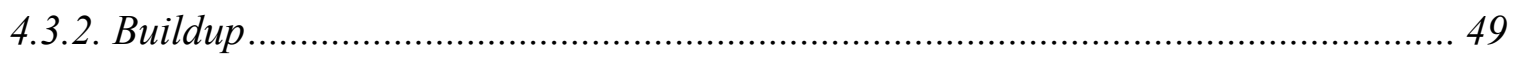

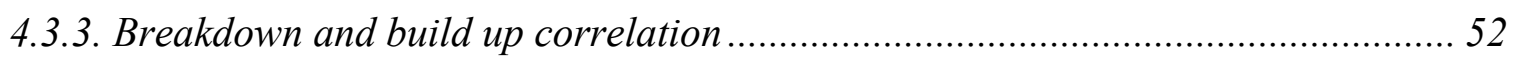

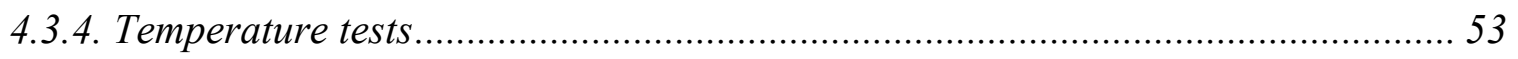

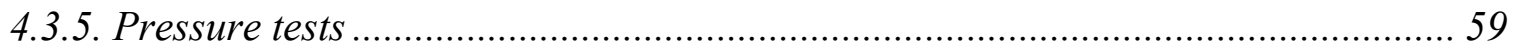

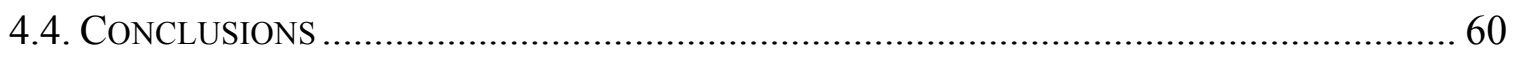




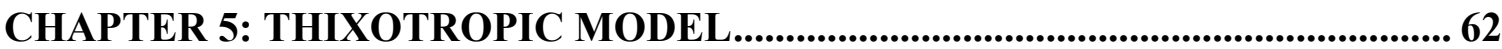

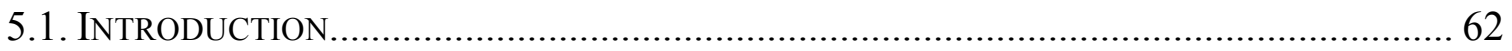

5.2. EXISTING THIXOTROPIC MODEL FOR CEMENTITIOUS MATERIALS.................................. 64

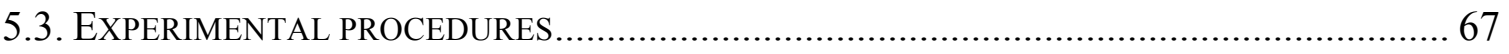

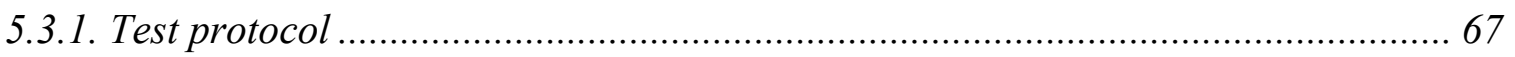

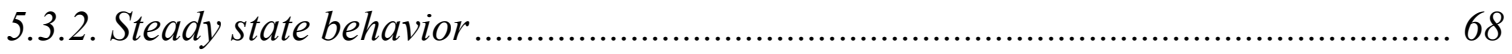

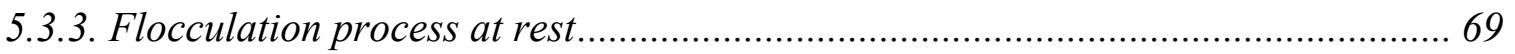

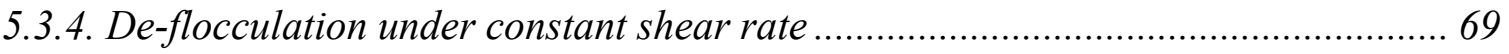

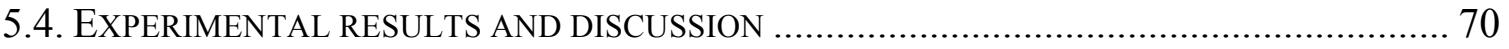

5.4.1. Flocculation process of cement pastes with different mix proportions ................. 70

5.4.2. De-flocculation process of cement pastes with different mix proportions.............. 75

5.4.3. Influence of temperature on flocculation and de-flocculation process of cement paste

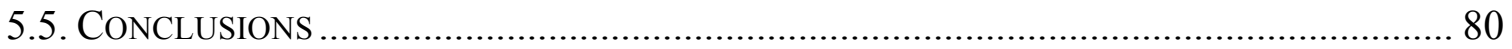

CHAPTER 6: CONCLUSIONS AND FUTURE WORK ............................................. 83

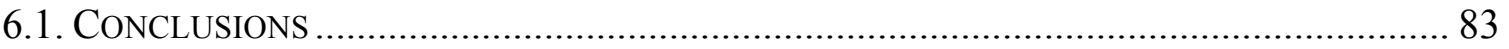

6.1.1. Influence of DE on fresh properties of cement pastes ......................................... 84

6.1.2. Influence of DE on thixotropic behavior of cement pastes ................................... 85

6.1.3. Influence of pressure and temperature on thixotropic behavior of cement pastes. 86

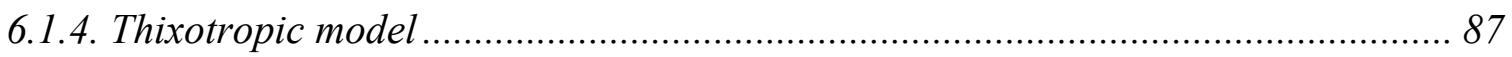




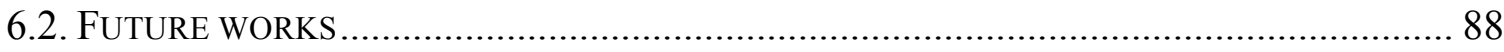

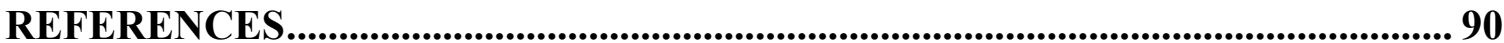

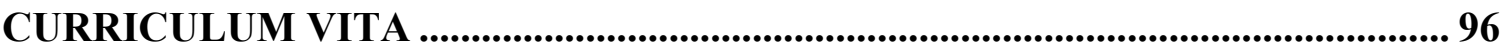




\section{LIST OF TABLES}

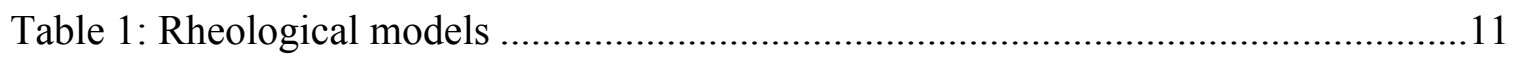

Table 2: Geometrical parameters of concentric cylinders ..........................................19

Table 3: Chemical composition of type I portland cement and Diatomaceous Earth .......23

Table 4: Mineral composition of type I Portland cement ...............................................24

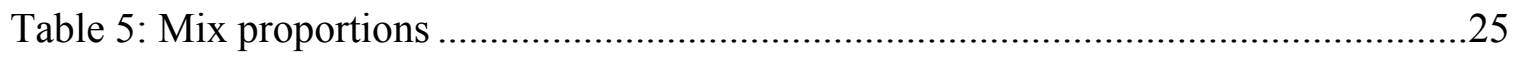

Table 6: Initial and final setting of cement pastes with different w/c ratios and DE

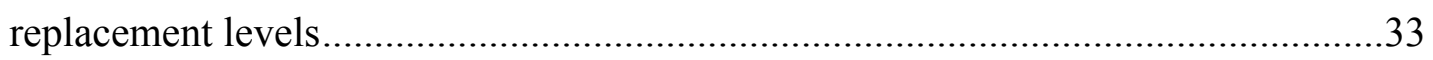

Table 7: Hydration age of first and second peaks of heat evolution rate for all cement pastes 


\section{LIST OF FIGURES}

Figure 1: relationship between rheological parameters in a two-plate model ................. 6

Figure 2: Flow curve of a Newtonian Fluid............................................................ 8

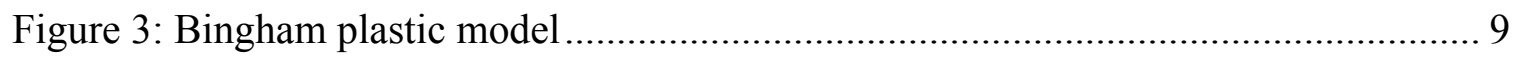

Figure 4: Some possible flow behavior of fluids ...................................................... 9

Figure 5: Time-dependent fluid under constant shear rate (Quanji, 2010) ...................... 10

Figure 6: Simple physical explanation of thixotropic behavior (Roussel, 2006) ............ 13

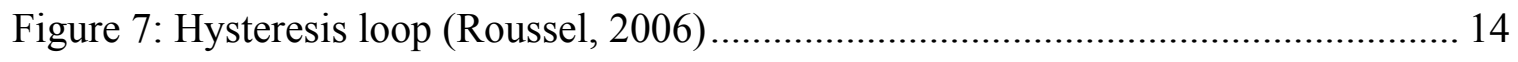

Figure 8: Multiple hysteresis loops for evaluating structural rebuilding rate ................. 15

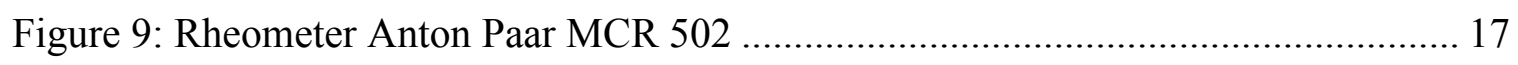

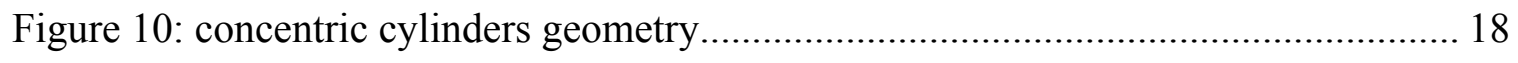

Figure 11: Pressure cell and magnetic coupling ...................................................... 18

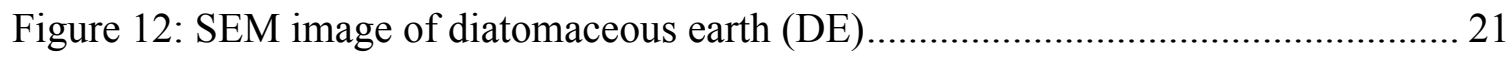

Figure 13: Viscosity of cement pastes against DE replacement levels......................... 27

Figure 14: Yield stress of cement pastes against DE replacement levels ...................... 27

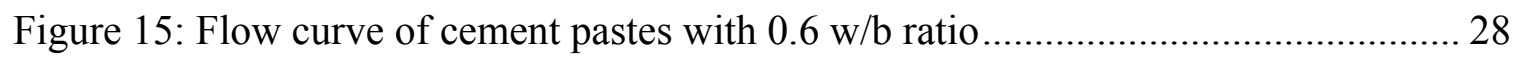

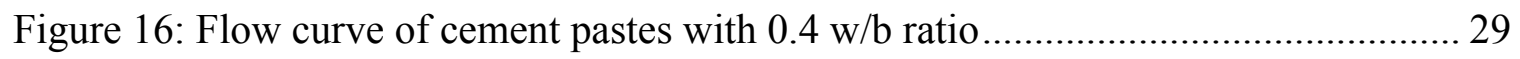

Figure 17: Flow diameter of cement pastes against DE replacement levels................... 30

Figure 18: Weight loss of cement pastes against time ............................................ 31

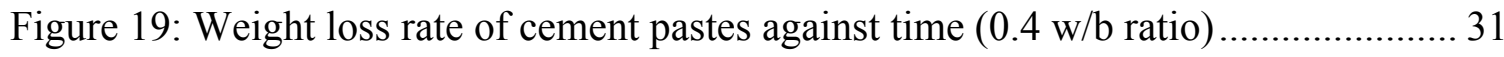




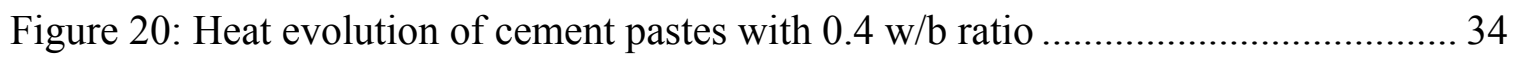

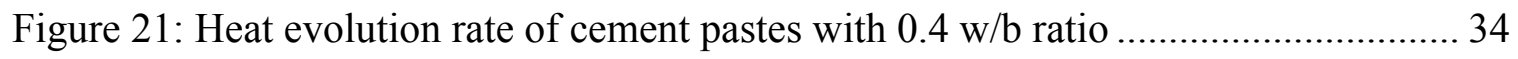

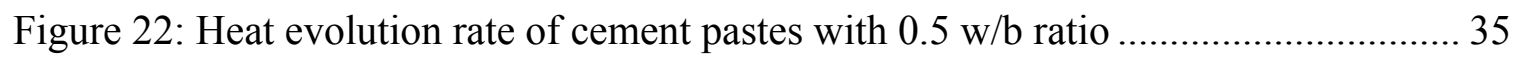

Figure 23: Heat evolution rate of cement pastes with $0.6 \mathrm{w} / \mathrm{b}$ ratio ............................. 35

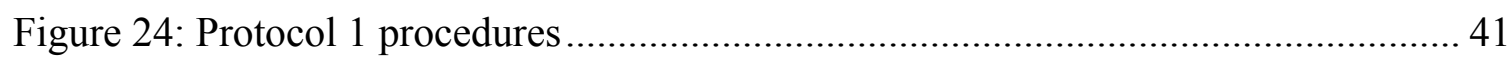

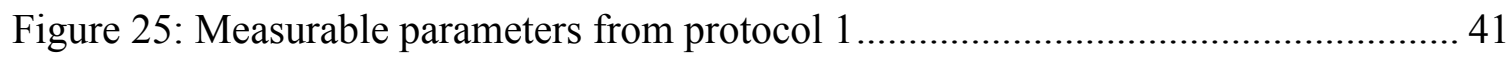

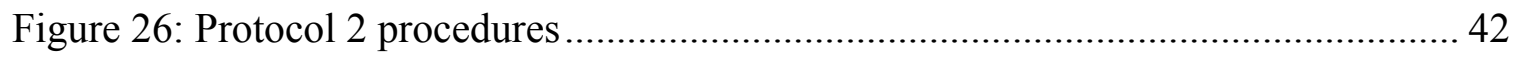

Figure 27: Measurable parameters from protocol 2 ............................................... 42

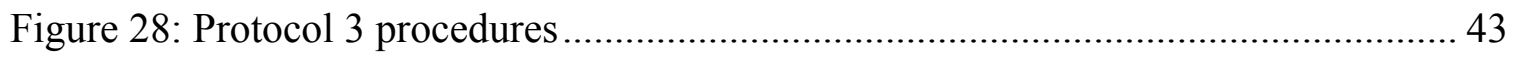

Figure 29: Measurable parameters from protocol 3 .............................................. 43

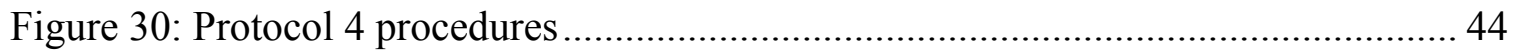

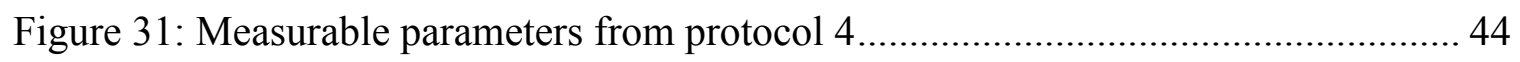

Figure 32: Breakdown energy against DE replacement level from protocol $1 \ldots \ldots \ldots \ldots \ldots . . .45$

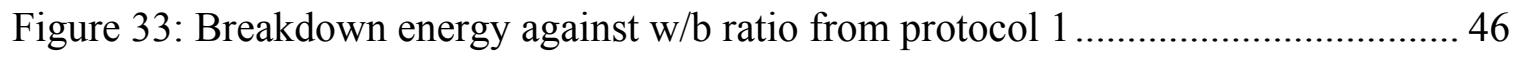

Figure 34: Drop in shear stress against DE replacement level for protocol 1 ................ 46

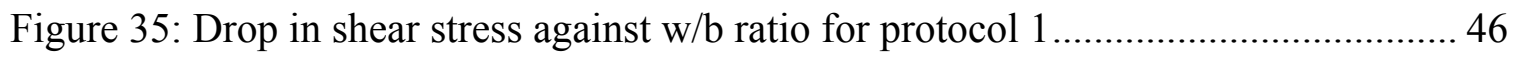

Figure 36: Time to reach to steady state against DE replacement level for protocol $1 \ldots . .46$

Figure 37: Time to reach to steady state against $\mathrm{w} / \mathrm{b}$ ratio for protocol 1 ...................... 47

Figure 38: Static yield stress against DE content from protocol 2 ............................ 48

Figure 39: Static yield stress against $w / b$ ratio from protocol $2 \ldots \ldots \ldots \ldots \ldots \ldots \ldots \ldots \ldots \ldots \ldots \ldots . . . . . \ldots \ldots$ 
Figure 40: Build-up ratios from protocol 3 against DE replacement levels ................... 50

Figure 41: Build-up ratios from protocol 3 against different $w / b$ ratios ......................... 50

Figure 42: Build-up time from protocol 3 against DE replacement levels .................... 51

Figure 43: Build-up ratios from protocol 4 against DE replacement levels .................... 52

Figure 44: Build-up ratios from protocol 4 against different $w / b$ ratios ........................ 52

Figure 45: Static yield stress from protocol 2 against DE replacement level $(\mathrm{w} / \mathrm{b}=0.5) . .54$

Figure 46: Static yield stress from protocol 2 against temperature $(\mathrm{w} / \mathrm{b}=0.5) \ldots \ldots \ldots \ldots \ldots . .54$

Figure 47: Static yield stress from protocol 2 against DE replacement level $(\mathrm{w} / \mathrm{b}=0.6) . .54$

Figure 48: Static yield stress from protocol 2 against temperature $(\mathrm{w} / \mathrm{b}=0.6) \ldots \ldots \ldots \ldots \ldots . . . .55$

Figure 49: Specific volume of water against temperature ....................................... 56

Figure 50: Build-up ration from protocol 4 against DE replacement level $(\mathrm{w} / \mathrm{b}=0.5) \ldots . .56$

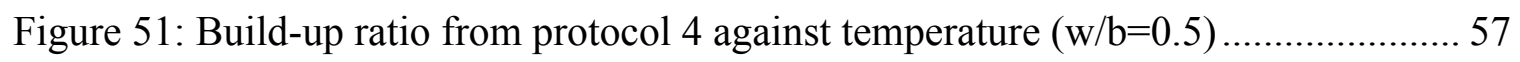

Figure 52: Build-up ratio from protocol 4 against DE replacement level (w/b=0.6) ...... 57

Figure 53: Build-up ratio from protocol 4 against temperature $(\mathrm{w} / \mathrm{b}=0.6) \ldots \ldots \ldots \ldots \ldots \ldots \ldots . . . . . \ldots 7$

Figure 54: Static yield stress for OPC-0.5 and OPC-0.6 at different temperatures against

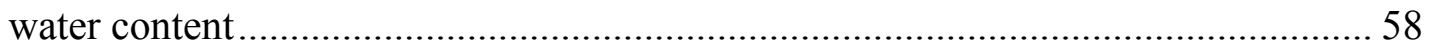

Figure 55: Build-up for OPC-0.5 and OPC-0.6 at different temperatures against water

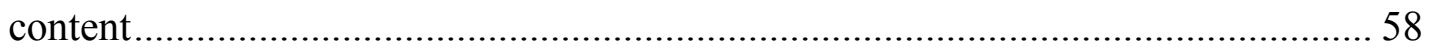

Figure 56: Static yield stress from protocol 2 at different pressures ............................. 60

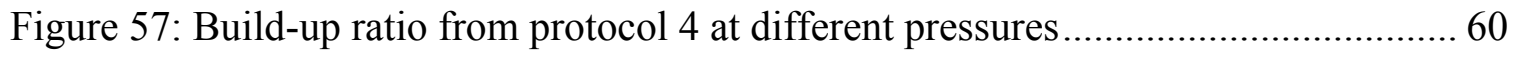


Figure 58: Examples of transient flow behaviors in practice against expected behavior from

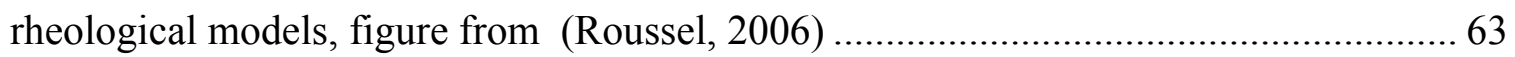

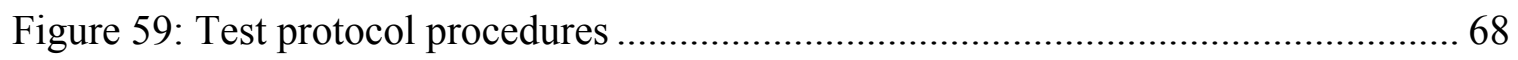

Figure 60: Example of fitted model to experimental data ....................................... 70

Figure 61 : Evolution of $\lambda 0$ by resting time for different mix proportions .................... 71

Figure 62: Evolution of $\lambda 0$ against DE replacement level at different resting times....... 72

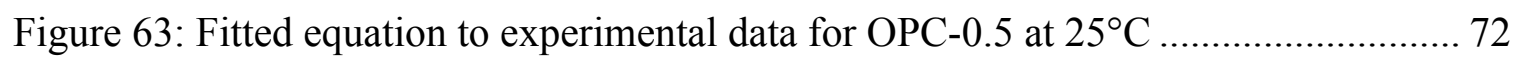

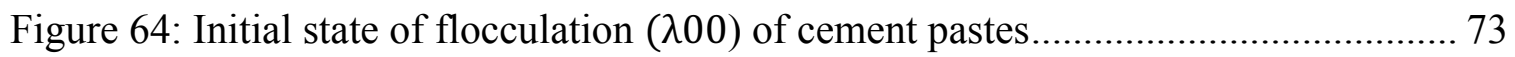

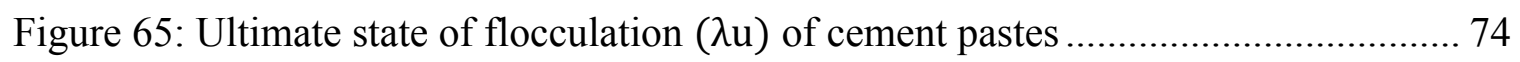

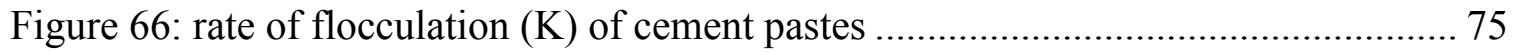

Figure 67: Evolution of $\alpha$ by resting time for different mix proportions....................... 76

Figure 68: Evolution of $\alpha$ against DE replacement level at different resting times......... 77

Figure 69: Evolution of $\lambda 0$ by resting times at different temperatures......................... 78

Figure 70: Evolution of $\lambda 0$ against temperature at different resting times .................... 78

Figure 71: Evolution of $\alpha$ by resting times at different temperatures ............................ 79

Figure 72: Evolution of $\alpha$ against temperature at different resting times ...................... 80

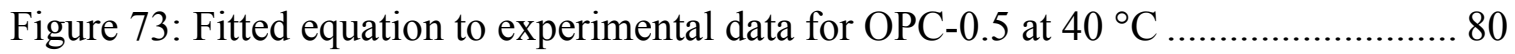




\section{CHAPTER 1}

\section{INTRODUCTION}

\subsection{Fresh and Rheological Properties of Concrete}

Concrete is the most widely used construction material all over the world. The value of ready mixed concrete produced in United States only is estimated at $\$ 35$ billion per year (NRMCA, 2016). This value shows a roughly 17\% growth in last three years (30 billion on 2013) and is expected to increase in upcoming years. Due to high application of concrete in construction industry, each year numerous studies are conducted to better understand its properties.

Concrete is a mixture of fine and coarse aggregates, usually sand, and either gravel or crushed stones, bonded together by a cementitious paste. The paste consists of cement, water and may also contains mineral or chemical admixtures. A freshly mixed concrete is so called "fresh concrete" and its properties are referred as fresh properties. During this period concrete is a mixture of liquid paste with solid aggregate suspensions. This allows concrete to be transported, placed and consolidated on a jobsite. This early stage is very important and has significant impact on the life time performance of the concrete. A poorly placed and compacted concrete will result in a concrete with poor hardened properties such as low strength and durability. Since transportation, placing, consolidating and finishing 
operation of concrete are closely related to its fresh properties, concrete should be designed in a way that its fresh properties meet the requirements of these intended application.

One of the most important fresh properties of concrete is workability. Workability is defined as the ease of placing, consolidating and finishing freshly mixed concrete. Thin and heavily reinforced concrete members require higher level of workability. A workable concrete should also meet the consistency and stability requirements. Consistency means the ability to flow and stability means resistance to segregation.

In fresh concrete, another phenomenon that can happen to its paste matrix is called bleeding. Bleeding is the development of a layer of water at the top or surface of freshly mixed concrete which is caused by sedimentation of solid particles and simultaneous upward movement of water. This phenomenon results in segregation, i.e. separation of solid particles (cement and aggregates) and water. High level of bleeding results in ununiformed material with a high water-to-binder ratio (w/b) at top layer that leads to week durability.

Once concrete is placed, setting and hardening happen. Setting time is another important fresh property of concrete that is closely related to construction. Initial setting time is considered as end of dormant period and start of transformation from a plastic, suspension-like mixture to a solid-form matrix. By start of setting period concrete has lost its workability and is not capable of being placed or consolidated anymore.

To better understand the concept of flowability, stability and other fresh properties of concrete, science of rheology is being widely used. Rheology studies deformation and flow of materials under shear stress. Rheology gives researcher, engineers, mix developers and others a more scientific approach to better understand and predict fresh properties of 
concrete. Currently, different rheological models are used to describe flow behavior of concrete. Among which the most common model is Bingham model. This model uses two rheological parameters, viscosity and yield stress, to describe flow behavior of materials. Viscosity is defined as resistance of material to flow, i.e. the rate of deformation of material under specific applied shear. Yield stress is the threshold between static and fluid behavior. Yield stress defines the minimum value of stress needed to be applied on a material to initiate its flow (Banfill, 2006). Another concept in rheology is thixotropy. It is a gradual decrease of viscosity under shear stress followed by a gradual recovery of structure when the stress is removed (Barnes, 1997). Some materials, including concrete show thixotropic behaviors. These concepts will be discussed in more details on next chapters.

\subsection{Research significant and objectives}

Concrete, when fresh, goes under various operations. It is normally transported to the job site, be placed and consolidated in the form work, etc. The easiness and quality of these operations are very much related to the intrinsic rheological properties of concrete. Therefore, a good understanding of how raw materials and mix design of concrete influence its fresh behavior under various conditions are needed. Current available standard testing methods for determining fresh properties of concrete such as slump, bleeding and setting time, are incapable of quantitatively characterize flow behavior of concrete under these conditions and only provide a simplified indication. Rheology provides a more scientific approach to better understand flow and setting behavior of concrete. It makes it possible to simulate the real in-situ conditions and observe how concrete behaves.

When at fresh state, the flowability of concrete is greatly determined by its paste matrix. Therefore, the research focus of this dissertation is the rheological properties of 
cementitious paste. Due to its heterogeneity, paste has intricate rheological behaviors and typically exhibits shear-thinning viscosity, yield stress, elasticity, and thixotropy. Thixotropic behavior of concrete can be a very good representative of its flow behavior on job site. For example, the breakdown aspect of thixotropy can help to explain how its flow behavior can impact pumping concrete and its buildup aspect can help to estimate the lateral force on formwork and concrete resistance to segregation. Thus, good understanding of thixotropic behavior of concrete can help to understand and predict flow behavior of concrete in practice.

The researchers have realized the importance of thixotropic behavior of concrete, however, there is no agreement on the measuring protocol of this parameter. This leads to the goal of this research, which is to propose a comprehensive series of protocols that can make quantitative measurements on both aspects of thixotropic behavior of concrete. While measuring breakdown and buildup separately, the correlations between these two aspects have also been investigated. On top of the developed measuring protocol, a new analytical model on thixotropic behavior of cement paste is proposed. With the proposed model, the influences of raw material characterizations on internal structural development in cement paste can be systematically studied.

For both the experimental and modeling work, real on-site conditions (such as pressure and temperature) influencing rheological properties are used.

\subsection{Structure of the thesis}

This thesis is divided into 6 chapters including introduction, rheology, fresh properties of cement pastes with different diatomaceous earth contents, thixotropic protocols, thixotropic model and conclusion and future work. 
Chapter 1 introduces the concept, research significant and structure of the thesis.

Chapter 2 provides a literature review based on basic rheology concepts, structural rebuilding rate and flocculation mechanism and thixotropy.

Chapter 3 studies fresh and rheological properties of cement pastes with different diatomaceous earth (DE) content.

Chapter 4 introduces the proposed thixotropic protocols to measure breakdown and buildup aspects of thixotropy.

Chapter 5 introduces the proposed thixotropic model.

Chapter 6 provides the key conclusions and future work. 


\section{CHAPTER 2}

\section{RHEOLOGY}

\subsection{Introduction}

Merriam Webster dictionary defines rheology as the science dealing with deformation and flow of matters. Rheology attempts to determine the intrinsic flow behavior of materials by studying the interrelationship between force, deformation and time. Most important rheological parameters are shear stress, shear rate and viscosity. To better illustrate the relationship between these parameters, a so called "two-plate model" is used (as show in Figure 1).

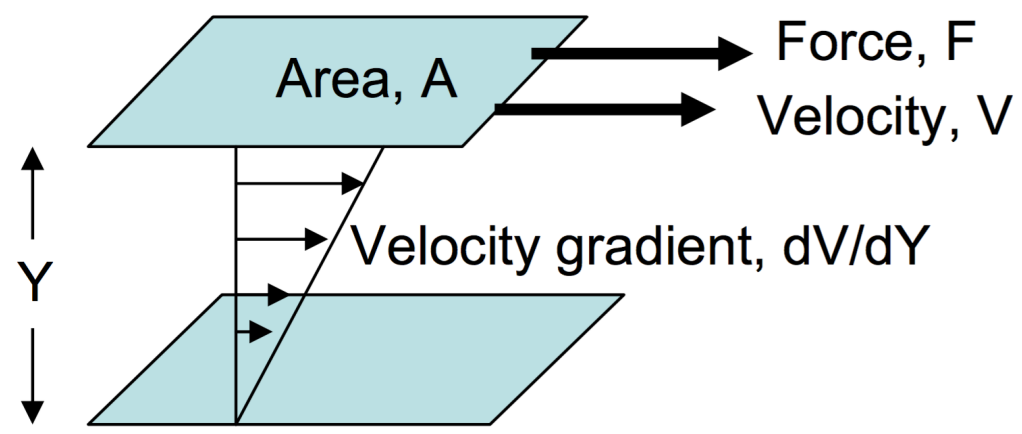

Figure 1: relationship between rheological parameters in a two-plate model (R. P. D. Ferron, 2008)

In this simple model, it is assumed that the material is confined between two parallel plates. The gap between two plates is Y. The bottom plate is stationary and top plate is moving with a constant velocity of $\mathrm{V}$. Thus, the flow velocity of material changes linearly 
from 0 at bottom plate to maximum velocity of $\mathrm{V}$ at top plate and shear rate (velocity gradient) can be mathematically shown as:

$$
\text { Shear rate }(\dot{\gamma})=\frac{\text { Velocity difference between two plates }(\mathrm{V})}{\text { gap between two plates }(\mathrm{Y})}
$$

Unit of shear rate is $1 / \mathrm{s}_{\text {or s }} \mathrm{s}^{-1}$.

Shear stress $(\tau)$ is shown as the applied force $(F)$ on top plate over its area (A):

$$
\tau=\frac{F}{\mathrm{~A}}
$$

Unit of shear stress is $\mathrm{N} / \mathrm{m}^{2}$ or Pa.

Viscosity is defined as resistance of material to flow and demonstrates the relationship between shear stress and shear rate. Based on Newton laws of flow, viscosity is simply shown as shear stress over shear rate:

$$
\mu=\frac{\tau}{\dot{\gamma}}
$$

And, its unit is Pa.s.

To better explain relationship between rheological parameters, shear rate and shear stress are usually plotted against each other in a graph. The resulting curve is called flow curve. Flow curve is very important to analyze the rheological properties of materials. Using flow curve and relationship between rheological parameters and time, materials can be classified into two groups; Newtonian fluids and non-Newtonian fluids (time independent and time dependent).

\subsubsection{Newtonian fluids}

Newtonian fluids follow Newton laws of flow, which considers a linear relationship between shear rate and shear stress, i.e. linear flow curve. Viscosity is the slope of the linear flow curve and therefore, it is constant. In other words, viscosity in Newtonian fluid is 
independent of flow conditions (shear stress, shear rate and time). Also, as shown in Figure 2, the flow curve passes the origin for a Newtonian fluid.

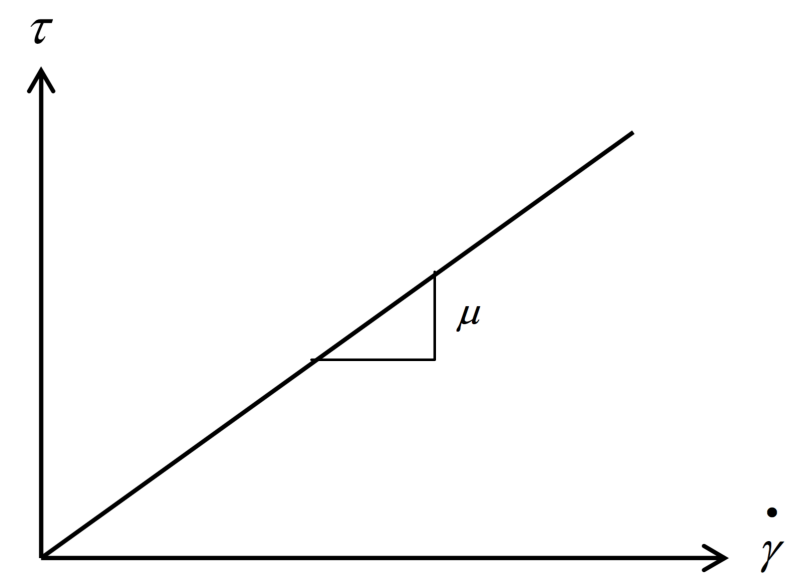

Figure 2: Flow curve of a Newtonian Fluid

Newtonian fluids are usually liquids with simple molecular formula and light molecular weight. Water, honey, oil, gasoline, alcohol and most solutions of simple molecules and diluted suspensions of solid particles with no or week inter-particle forces are Newtonian fluids.

\subsubsection{Non-Newtonian fluids}

Unlike Newtonian fluids, non-Newtonian fluids do not show a linear relationship between shear rate and shear stress which goes through the origin. This class of fluids either show a non-linear flow curve, a yield stress or both. Yield stress $\left(\tau_{0}\right)$ is another important rheological property. It is defined as minimum shear stress which is needed to be applied on a material to make it flow. Some non-Newtonian fluid do not flow till shear stress reaches to a certain value (yield stress) (as shown in Figure 3). Also, non-Newtonian fluid do not necessarily show a constant viscosity at different shear rates. It means viscosity varies with each given shear rate. Different non-Newtonian fluids can show different flow 
behaviors. Shear thinning fluids show a decline in viscosity by increase in shear rate. On the other hand, Shear thickening fluids show higher viscosity by increase in shear rate. Figure 4 shows some possible flow behaviors of fluids. For these type of materials, viscosity needs to be measured at each specific shear rate. In this case, viscosity at a specific shear rate is the slope of the line passes through the corresponding point on flow curve and the origin, and it is called apparent viscosity.

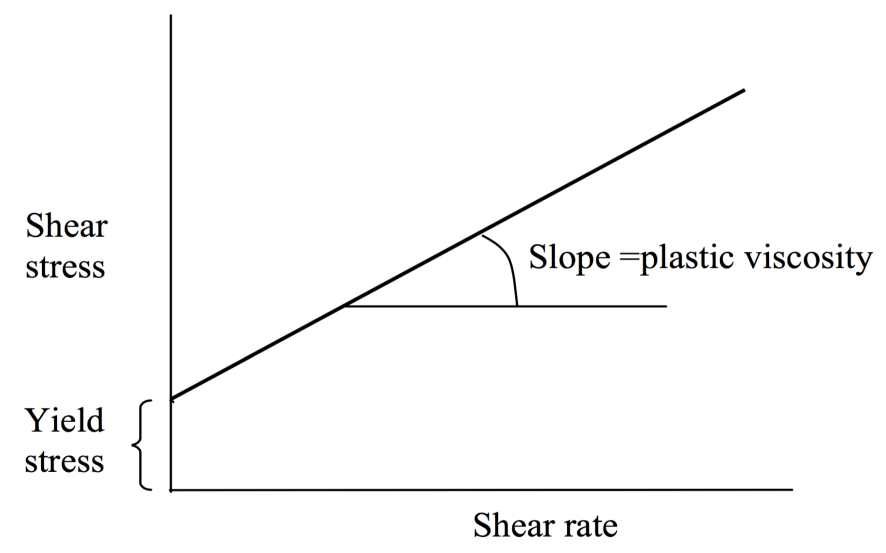

Figure 3: Bingham plastic model

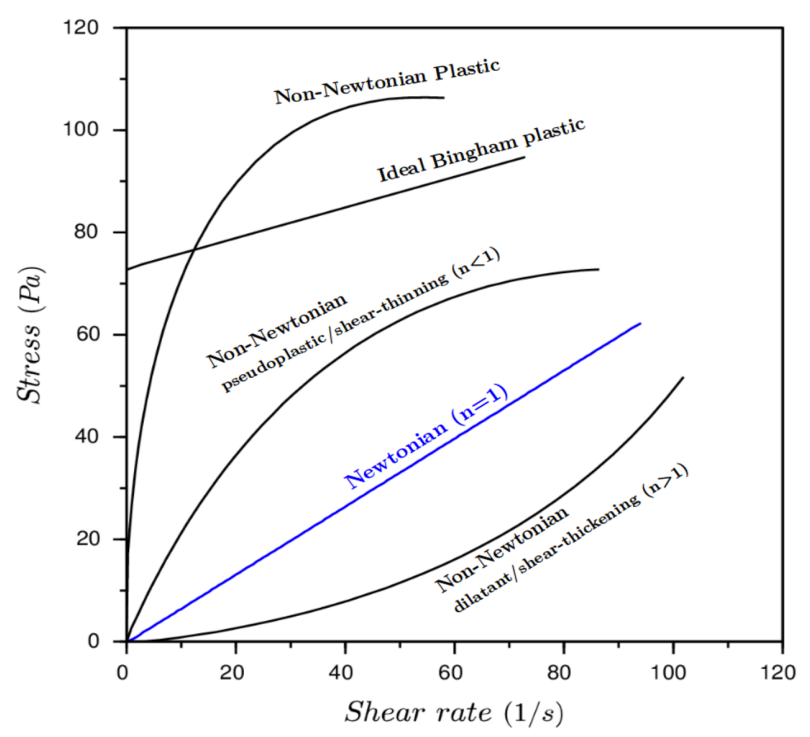

Figure 4: Some possible flow behavior of fluids (Figure from https://www.simscale.com/docs/content/simulation/model/materials/OF Non-Newtonain-Models.html?highlight=non\%20newtonian) 
Non-Newtonian fluids can also be divided into two different groups; time-independent and time-dependent. For a time-independent fluid, viscosity is independent of time. It means at any given shear rate these materials display a single viscosity and this viscosity does not change with time. On the other hand, for a time-dependent fluid, viscosity at any given shear rate changes with time. In other words, if a constant shear rate is applied on time-dependent materials, the value of shear stress and consequently viscosity will change by time (see Figure 5). However, keeping this constant shear rate long enough will eventually lead to an equilibrium condition where shear stress and viscosity become constant.

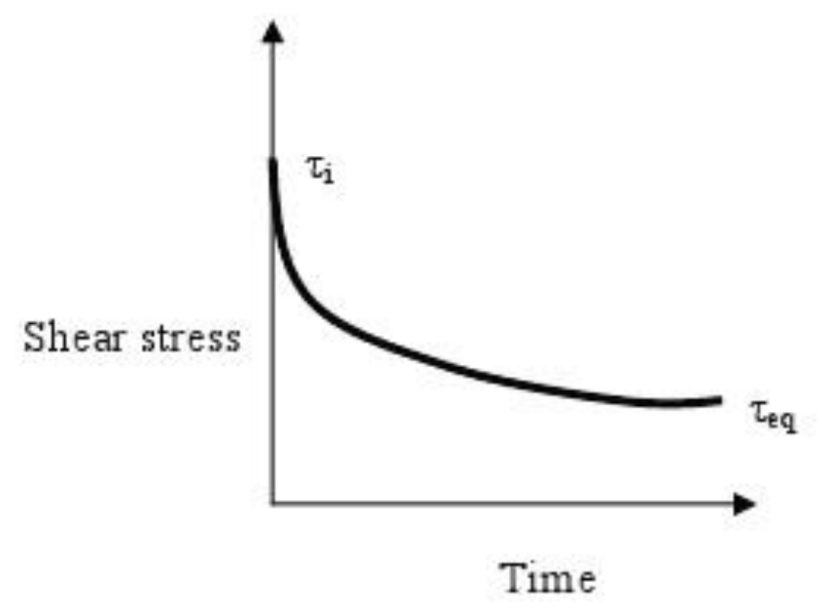

Figure 5: Time-dependent fluid under constant shear rate (Quanji, 2010)

Non-Newtonian fluids are usually complex mixtures and suspensions with high concentrated solid particles and high inter-particle forces such as slurries, pastes (including cementitious pastes), gels, polymer solutions, etc. 


\subsubsection{Rheological models}

Rheological models are proposed to describe flow behavior of materials. These models use different mathematical forms to best fit the flow curve obtained by experimental measurements. A list of some of common rheological models are presented in Table 1. Bingham model uses linear regression to find yield stress and plastic viscosity. Due to its simplicity, it is the most commonly used model to study the cementitious materials. Herschel-Buckley is another common model for cementitious materials. Unlike Bingham, this model uses nonlinear regression. The flow index (n) in Herschel-Bulkley model is used as an indication of the shear behavior. When flow index is smaller than 1 , the material exhibits shear thinning behavior, and when it is larger than 1, material shows shear thickening behavior.

Table 1: Rheological models

\begin{tabular}{cc}
\hline Model & Equation \\
\hline Newtonian & $\mu=\frac{\tau}{\dot{\gamma}}$ \\
Bingham & $\tau=\tau_{0}+\mu_{P} \cdot \dot{\gamma}$ \\
Power-law & $\tau=K \cdot \dot{\gamma}^{n}$ \\
Herschel-Bulkley & $\tau=\tau_{0}+K \cdot \dot{\gamma}^{n}$ \\
Casson & $\sqrt{\tau}=\sqrt{\tau_{0}}+\sqrt{\mu_{\mathrm{P}}} \times \sqrt{\dot{\gamma}}$ \\
\hline
\end{tabular}




\subsection{Thixotropy}

Thixotropy is defined as the continuous decrease of viscosity with time when flow is applied to a sample that has been previously at rest and the subsequent recovery of viscosity in time when the flow is discontinued (Mewis \& Wagner, 2009). From this definition, two conclusions can be drawn. First, thixotropic materials are non-Newtonian (time independent) which exhibits shear thinning behavior and therefore, their shear history impact their thixotropic behavior. Second, thixotropy is a reversible process. Thixotropic materials, due to their microstructure, show complex rheological behavior. These materials are usually suspensions with high concentrated solid particles. The inter-particle forces between solid particles such as van der Waals attraction, electrostatic repulsion and steric hindrance result in formation of flocs which normally evolve into a space-filling particulate network (Wallevik, 2009). The inter-particle bonds are, however, weak enough to be broken by the mechanical stresses that occur during flow. The result is that during flow the network breaks down in separate flocs, which decrease further in size when the shear rate is increased. However, reduce or stop in the shear rate can cause a growth of the flocs; i.e. the particulate network will rebuild at rest or lower shear rates.

A simple physical explanation for thixotropic behavior is given by Roussel (Roussel, 2006). The particle interaction forces determine a potential energy well for each particle. Depth of this energy well represent the minimum value of energy required to set the particle free (see Figure 6). As long the applied energy is below this value, the particle will not be able to leave. As soon as applied energy exceeds this minimum value, the particle can get out of the well and the flow occurs. If the flow continues long enough, materials reaches to an equilibrium state, i.e. depth of energy well will decrease to a minimum 
amount. Again, as soon as applied energy falls below the minimum value (in other words, shear rate is decreased or material is at rest), the particle goes back to well and depth of energy well starts to increase to get back to its original state.

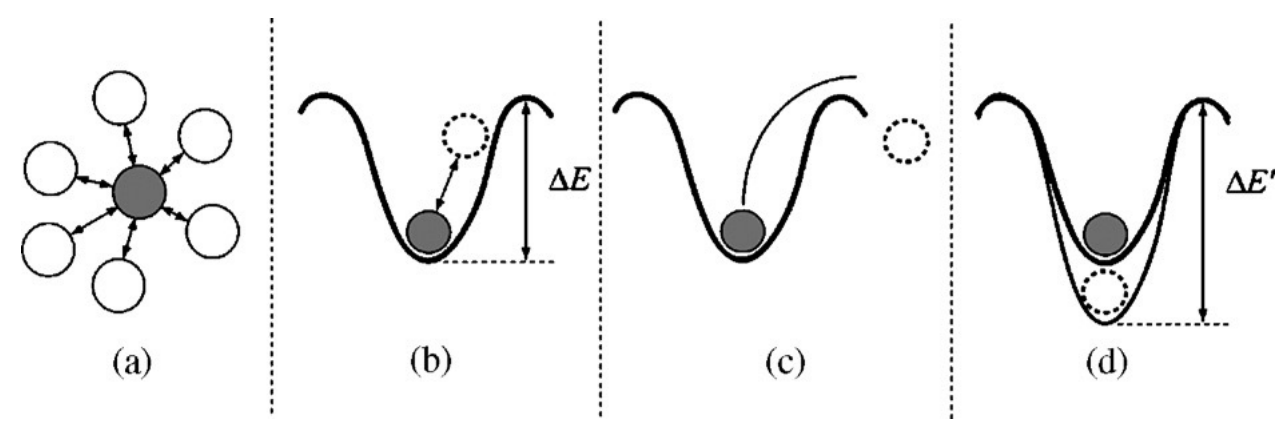

Figure 6: Simple physical explanation of thixotropic behavior (Roussel, 2006)

\subsubsection{Current methods of measuring thixotropy of cementitious materials}

Hysteresis loop has been the most common method of measuring thixotropy of cementitious materials in last few decades. In this method, the area between two subsequent flow curves, an up-curve followed by a down-curve, is measured and linked to thixotropic characterization of material. During the up-curve, shear rate is gradually increased from 0 to a maximum value which breaks down part of the already formed structure. Then, in down curve, the shear rate is gradually reduced from the maximum value back to 0 , which let the sample to rebuild part of its breakdown structure. The idea is, the area between these two curves represents the work done per unit time and unit volume of the sample to break some of the initially present linkages. 


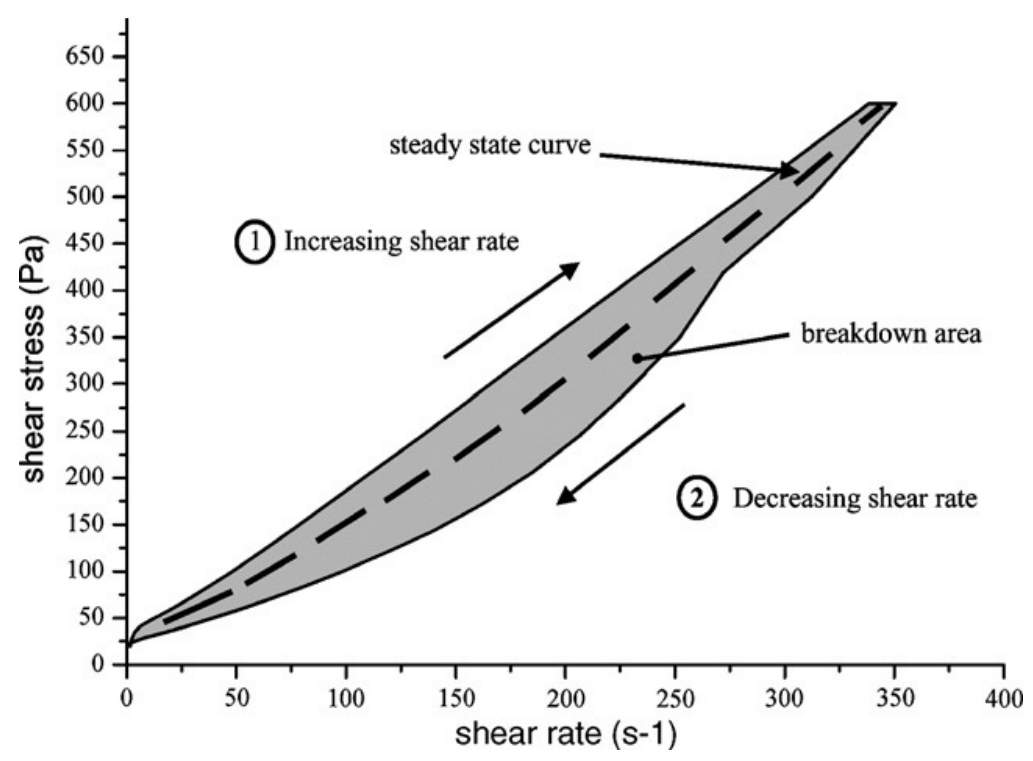

Figure 7: Hysteresis loop (Roussel, 2006)

Although hysteresis loop is a quick quantitative method, it does not give an intrinsic value of any physical rheological parameters. There is thus, no possibility apart from empirical correlations to use the measured result in the study of thixotropic behavior of materials. Also, there are other problems associated with this method. Firstly, the shear history of the sample, resulted from mixing, transporting and even placing sample in equipment, significantly influences the test results. Secondly, in this method, either in up-curve or down-curve, sample does not reach to an equilibrium condition. Thus, test results are very dependent on experimental conditions such as maximum shear rate value and rate of increase and decrease in shear rate. Moreover, in hysteresis loop time and shear rate change simultaneously which makes it less suitable to separate the influence of these two parameters. And finally, the measured area between hysteresis loop's flow curves, couples the influence of two aspects of thixotropy, i.e. buildup and breakdown. Therefore, a sample with high breakdown and high buildup rate can have the same hysteresis loop area as another sample with low breakdown and low buildup rate, while they behave very differently in practice. 


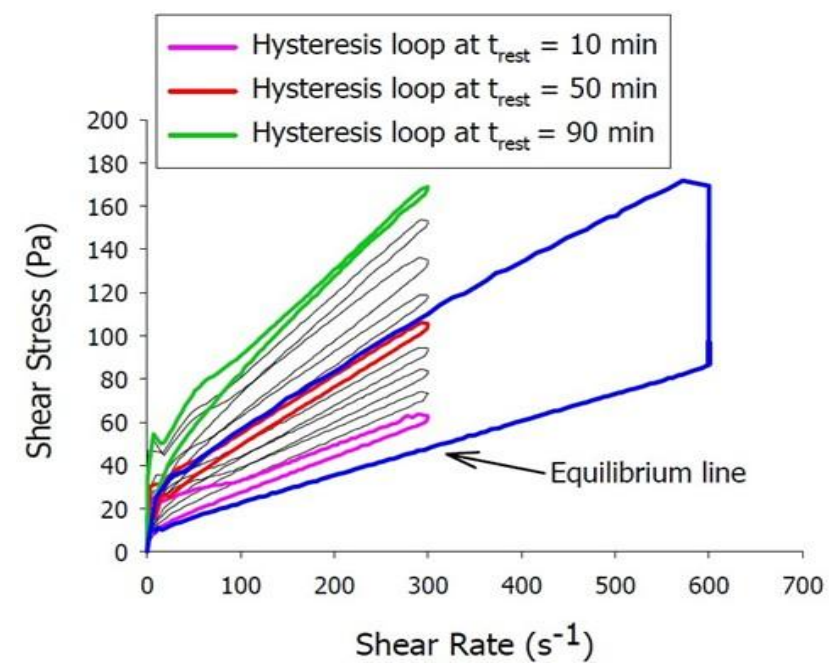

Figure 8: Multiple hysteresis loops for evaluating structural rebuilding rate (R. P. D. Ferron, 2008)

Some modified methods based on hysteresis loop has been proposed in a try to address and minimize its deficiencies. For example, Ferron et. al (R. P. Ferron, Gregori, Sun, \& Shah, 2007) suggested a multiple hysteresis loop method. In this method, first, an equilibrium loop is conducted on the sample. In this equilibrium loop, high shear rate at the end of up-curve is maintained long enough to sample reaches an equilibrium condition, and then it is followed by the down curve, i.e. equilibrium line. Then, successive hysteresis loops at different resting times are conducted and the area between the up-curve of each hysteresis loop and the equilibrium line (specific rebuilding energy) is measured and plotted against resting time. The slope of this graph is considered as rate of rebuilding and used to compare rebuilding rate of different mixtures. Although, this method, by using equilibrium loop, minimizes some flaws of single hysteresis loop such as the influence of shear history from mixing, transporting, etc. But it fails to address the influence of shear history of each hysteresis loop on the subsequent loops. Also, it only focuses on rebuilding aspect of thixotropy. Therefore, more reliable and comprehensive methods to quantitatively measure thixotropy are needed. 


\subsubsection{Factors influencing rheology of cementitious materials}

Many different factors may influence rheological properties of concrete. Some of the main factors are mix proportions, shape and texture of aggregates, chemical and mineral admixtures, mixing, transportation, placing and pumping procedure, ambient condition, elapsed time etc. As mentioned earlier, this research is focused on rheological properties of cementitious pastes. Thus, the following parameters are studied:

- Water content: cement pastes with different water content are considered for this research $(0.4,0.5$ and $0.6 \mathrm{w} / \mathrm{b}$ ratios $)$.

- Admixtures: a natural pozzolanic admixture, diatomaceous earth (DE), is used as partial replacement for cement at different replacement levels $(2,6$ and $10 \%)$.

- Temperature: to simulate real in-situ ambient temperature, rheological properties at different temperatures are studied $\left(10,25\right.$ and $\left.40{ }^{\circ} \mathrm{C}\right)$.

- Pressure: to simulated applied pressure on cementitious pastes at different pumping rates rheological test at three different pressures are conducted $(0$, 10 and $20 \mathrm{MPa})$.

- Elapsed time: rheological properties at different resting times are measured.

To study influence of each separate parameter and avoid coupled influence on rheological properties, each parameter is studied while other variables are kept constant.

\subsection{Equipment and testing methods}

A state of the art rheometer, Anton Paar MCR 502, is used to conduct rheological tests (see Figure 9). This rheometer can control both shear stress and shear rate. In other 
words, this rheometer is capable of applying shear rate and monitoring shear stress and vice versa. It gives the researchers the opportunity to perform wide range of rheological tests. Also, it is capable of controlling temperature from room temperature up to $400{ }^{\circ} \mathrm{C}$. For temperatures below room temperature, a water bath can be connected to the rheometer to achieve desired temperature as shown in Figure 9.

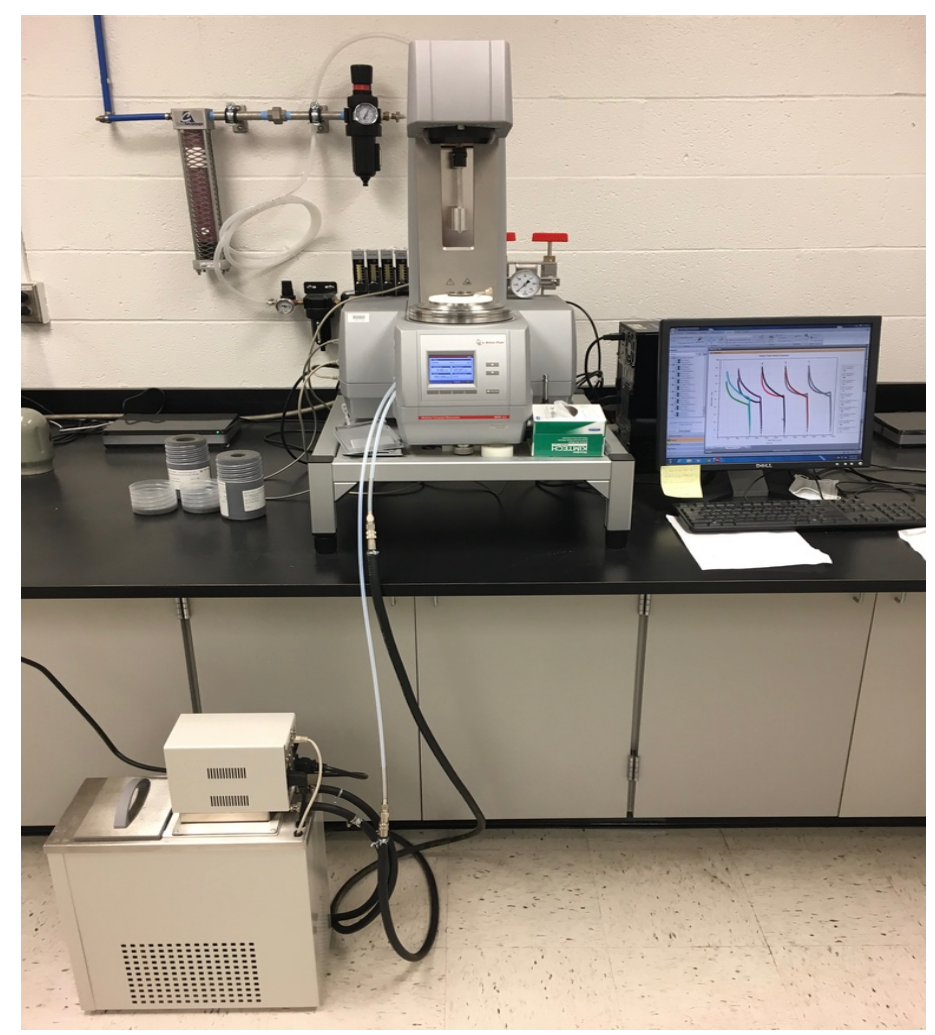

Figure 9: Rheometer Anton Paar MCR 502

For purpose of this study, concentric cylinders with conical end geometry (cup and bob) is selected. This geometry makes placing cementitious pastes in rheometer more convenient and prevent water evaporation and water loss during test. Schematic and real picture of cup and bob in use are shown in

Figure 10. 

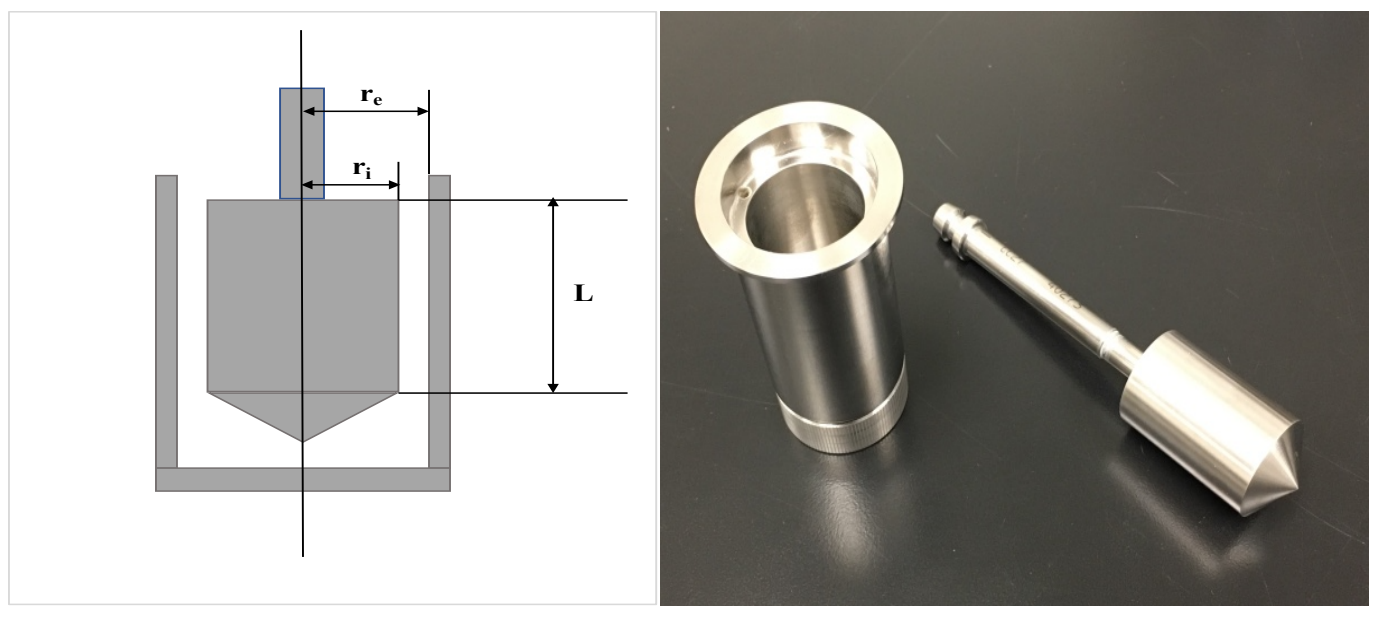

Figure 10: concentric cylinders geometry
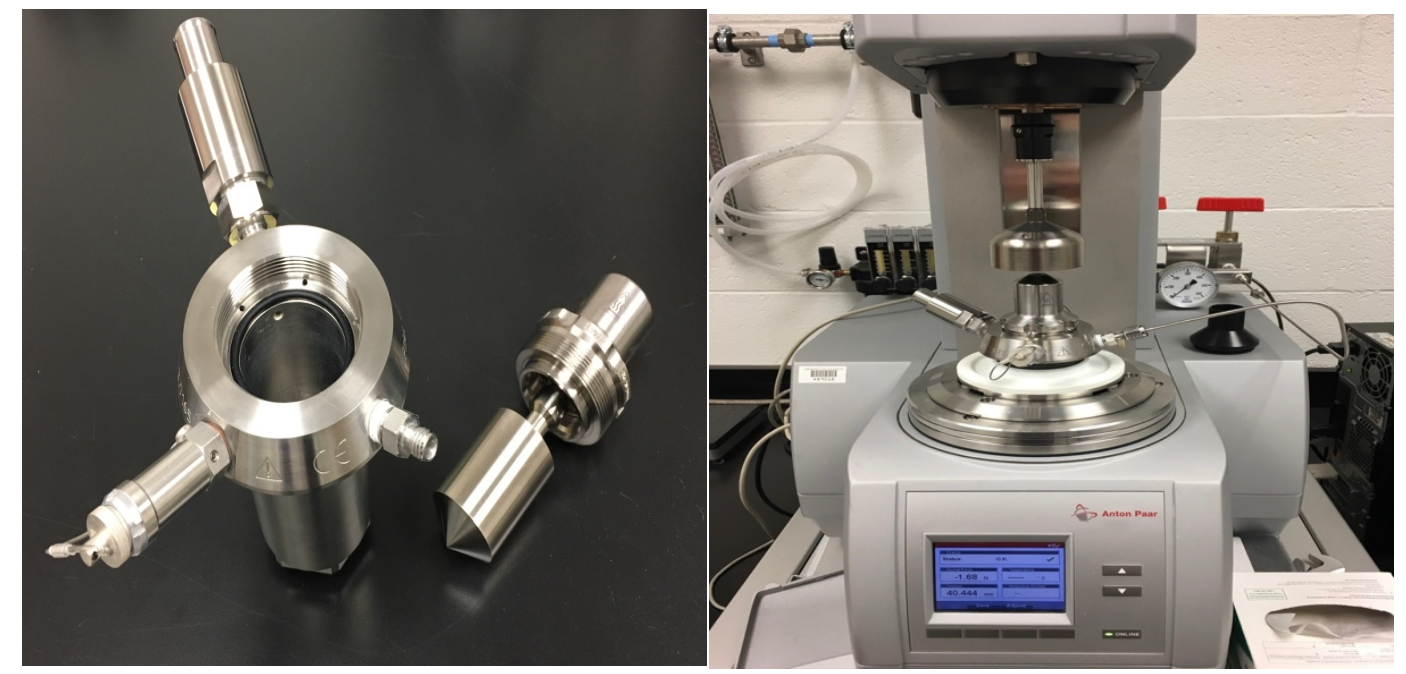

Figure 11: Pressure cell and magnetic coupling

For rheological tests at high pressures, a pressure cell is used. The pressure cell has almost the same geometry as regular cup and bob (as shown in Figure 11). In pressure tests, after the sample is placed, the pressure cell is tightly capped using high strength bolt. Then, the desired pressure is achieved by pumping compressed Nitrogen gas into the cell. In the pressure cell, there is no physical contact between the inner cylinder (bob) and the rotor 
and a magnetic holder is used to transfer torque to the bob. Through this magnetic coupling, the desired shear is applied on the sample.

Gap size and other geometrical parameters of both regular and pressure cell cup and bob are listed in Table 2 .

Table 2: Geometrical parameters of concentric cylinders

\begin{tabular}{ccc}
\hline Geometrical parameters & Regular cup and bob & Pressure cell cup and bob \\
Gap size $\left(\mathrm{r}_{\mathrm{e}}-\mathrm{r}_{\mathrm{i}}\right)$ & $1.130 \mathrm{~mm}$ & $1.401 \mathrm{~mm}$ \\
$\mathrm{r}_{\mathrm{e}}$ & $14.460 \mathrm{~mm}$ & $18.000 \mathrm{~mm}$ \\
$\mathrm{r}_{\mathrm{i}}$ & $13.330 \mathrm{~mm}$ & $16.599 \mathrm{~mm}$ \\
$\mathrm{~L}$ & $39.997 \mathrm{~mm}$ & $120^{\circ}$ \\
Cone angle & $120^{\circ}$ & \\
\hline
\end{tabular}




\section{CHAPTER 3}

\section{FRESH PROPERTIES OF CEMENT PASTES WITH DIFFERENT DIA- TOMACEOUS EARTH CONTENTS}

\subsection{Introduction}

Diatomaceous earth (DE), also known as Kieselguhr, is a chalklike, soft, very fine grained, earthy, siliceous sedimentary material (Robert D. Crangle, 2013). It is finely porous, less in density, essentially chemically inert with low thermal conductivity (Bakr, 2010; Robert D. Crangle, 2013). DE consists of amorphous hydrous silica cell walls of dead diatoms (opal, $\mathrm{SiO}_{2} \bullet \mathrm{nH}_{2} \mathrm{O}$ ), which are microscopic single-cell aquatic plants (algae). The diatoms cells contain an internal, elaborate siliceous skeleton consisting of two valves (frustules), which fit together much like a pillbox (Dolley, 1999). These skeletons vary in size from less than $1 \mu \mathrm{m}$ to more than $1 \mathrm{~mm}$, but are typically from 10 to $200 \mu \mathrm{m}$ (Robert D. Crangle, 2013). There are many different types of DE but almost all commercial DEs are composed of around $80 \%$ to $90 \%$ silica $\left(\mathrm{SiO}_{2}\right)$ (Bakr, 2010; Jud Sierra, Miller, Sakulich, MacKenzie, \& Barsoum, 2010; Miller, Sakulich, Barsoum, \& Jud Sierra, 2010).

United States' production of diatomite in 2015 is estimated to be 925,000 metric tons, which is more than $40 \%$ of whole world's DE production (Sally Jewell \& Kimball, 2016). There are wild ranges of applications for DE. In 2015 in US, 55\% of diatomite was used in filter aids, $21 \%$ was used as cement additives, 14\% was used as fillers, and 9\% was 
used as absorbents. According to United States Geology Surveys (USGS), using DE as cement additives has increased significantly from $14 \%$ in 2014 to $21 \%$ in 2015 and now it is the second largest DE consumption in US (Sally Jewell \& Kimball, 2015, 2016).

DE has been used from thousand years ago as an addition to lime for binding limestone aggregates together in ancient Egypt pyramids to more recent years as a natural pozzolanic additive for concrete (Jud Sierra et al., 2010; Miller et al., 2010). It is believed that replacing Portland cement with DE to some levels can improve some properties of concrete.

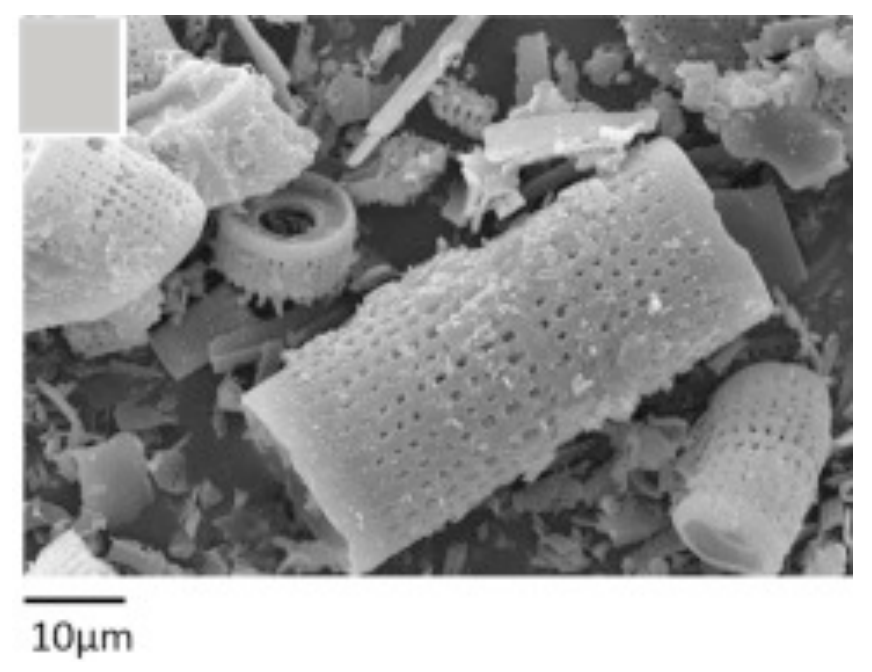

Figure 12: SEM image of diatomaceous earth (DE) (Mejía, de Gutiérrez, \& Montes, 2016)

Because of its unique morphology, porous structure and high water absorption level, DE is excepted to influence properties of concrete especially its fresh properties (Figure 12). Being different from other commonly used pozzolanic materials, such as fly ash, silica fume, and slag, that have tons of researches been conducted to investigated their influences on concrete properties, very few researches have been reported on the application of DE in concrete. In recent years, due to the increased usage of DE as cement additive, 
some researches focused on mechanical properties have been reported (Aydin \& Gül, 2007; Degirmenci \& Yilmaz, 2009; Ergün, 2011; Fragoulis, Stamatakis, Papageorgiou, \& Chaniotakis, 2005; Kastis, Kakali, Tsivilis, \& Stamatakis, 2006; Stamatakis, Fragoulis, Csirik, Bedelean, \& Pedersen, 2003; Y1lmaz \& Ediz, 2008). Separate studies by Ergun (Ergün, 2011), Fragoulis et al. (Fragoulis et al., 2005) and Stamatakis et al. (Stamatakis et al., 2003) on different types of diatomaceous earth showed that replacing cement by DE up to $10-15 \%$ increases compressive and flexural strength compare to control samples (almost at all different measured ages with highest increase at 10\%). They concluded that samples with higher content of reactive silica and higher Blaine surface show higher increase in compressive strength. Also, studies by Kastis et al. (Kastis et al., 2006) and Yilmaz and Ediz (Y1lmaz \& Ediz, 2008) found that replacement levels up to 10\% show comparable compressive strength compare to control samples, while samples with higher DE content show a significant drop in strength. On the other hand, studies by Aydin and Gül (Aydin \& Gül, 2007) and Degirmenci and Yilmaz (Degirmenci \& Yilmaz, 2009) showed that for all replacement levels and all measured ages module of elasticity and compressive and flexural strength were lower than control samples. However, they found that using DE can improve both freeze-thaw durability and sulphate attack resistance. As these studies mainly focus on hardened properties of concrete, a comprehensive study on impact of DE on fresh properties of concrete is lacking. Fresh properties of concrete, including setting, rheology, bleeding and early hydration are closely related to operation and construction, such as transporting, placing, compacting etc.; and they also have great impact on the life time performance of concrete. 
Therefore, this chapter studies the influence of DE as partial replacement of Portland cement on fresh properties of cement pastes. For this purpose, pastes with water-tobinder ratios $(\mathrm{w} / \mathrm{b})$ of $0.4,0.5$ and 0.6 were investigated. Based on findings of aforementioned studies, only replacement levels up to $10 \%$ were considered. Thus, for each $\mathrm{w} / \mathrm{b}$ ratio, three replacement levels of $2 \%, 6 \%$, and $10 \%$ of cement with DE were used. The influences of DE replacement on flowability, viscosity and yield stress, bleeding, setting time and heat signature were studied systematically.

\subsection{Raw materials}

Ordinary Type I Portland cement (from CEMEX, Louisville plant (KY, USA)) was used in all the mixtures. The mean size of the cement particles was $11.4 \mu \mathrm{m}$, determined through a laser particle size analyser. The Blaine surface area was $400.8 \mathrm{~m}^{2} / \mathrm{kg}$. The chemical compositions of this type I cement were measured with XRF and they are listed in Table 3. The mineral compositions for this cement are calculated based on the Bougue's equation (Taylor, 1997) and are provided in Table 4.

DE used in this study was a commercial pure diatomaceous earth usually powder available widely on the market. The mean size of DE particles is $19 \mu \mathrm{m}$ based on a laser particle size analyzer. And the Blaine surface area was $593 \mathrm{~m}^{2} / \mathrm{kg}$. The chemical composition of DE is also listed in Table 3.

Table 3: Chemical composition of type I portland cement and Diatomaceous Earth

\begin{tabular}{ccc}
\hline Compound (\%) & Type I Portland cement & Diatomaceous Earth \\
\hline $\mathrm{SiO}_{2}$ & 19.70 & 93.5 \\
$\mathrm{Al}_{2} \mathrm{O}_{3}$ & 4.84 & 1.6 \\
$\mathrm{Fe}_{2} \mathrm{O}_{3}$ & 3.05 & 1.1 \\
$\mathrm{CaO}$ & 62.62 & 0.4 \\
$\mathrm{MgO}$ & 4.00 & 0.05
\end{tabular}




\begin{tabular}{ccc}
$\mathrm{SO}_{3}$ & 3.23 & 0.12 \\
$\mathrm{Na}_{2} \mathrm{O}$ & 0.15 & 2.51 \\
$\mathrm{~K}_{2} \mathrm{O}$ & 0.49 & 0.09 \\
$\mathrm{LOI}$ & 1.21 & 0.35 \\
\hline
\end{tabular}

Table 4: Mineral composition of type I Portland cement

\begin{tabular}{cc}
\hline Compound & Wight, \% \\
\hline $\mathrm{C}_{3} \mathrm{~S}$ & 59.1 \\
$\mathrm{C}_{2} \mathrm{~S}$ & 11.9 \\
$\mathrm{C}_{3} \mathrm{~A}$ & 7.67 \\
$\mathrm{C}_{4} \mathrm{AF}$ & 9.3 \\
\hline
\end{tabular}

1.9. Mixing procedures and testing matrix

The details of mix proportions are listed in Table 5, where "OPC" indicates control samples with ordinary type I cement only, and "D" indicates the pastes with DE replacement. Three $\mathrm{w} / \mathrm{b}$ ratios of $0.4,0.5$, and 0.6 were used. For each $w / b$ ratio, three replacement levels of DE were studied.

When mixing the control pastes (OPC-0.4, OPC-0.5 and OPC-0.6), water was gradually added to the cement over the first minute of mixing and then continued to mix for 2 additional minutes at a low speed of $136 \mathrm{rpm}$. The sample was then allowed to rest for 2 minutes, which was followed by another 3 minutes of mixing at high speed of $195 \mathrm{rpm}$.

For cement pastes with DE replacement, cement powder and DE were firstly dry mixed for a minute with low speed of $136 \mathrm{rpm}$. Then, the same mixing procedure used for control pastes was followed. Mixing and all following tests were conducted at $25^{\circ} \mathrm{C}$. 
Table 5: Mix proportions

\begin{tabular}{cccccc}
\hline Cement paste & w/b & DE replacement level (\%) & Cement (gr) & DE (gr) & Water (gr) \\
\hline OPC-0.4 & 0.4 & 0 & 700 & 0 & 280 \\
D2-0.4 & 0.4 & 2 & 686 & 14 & 280 \\
D6-0.4 & 0.4 & 6 & 658 & 42 & 280 \\
D10-0.4 & 0.4 & 10 & 630 & 70 & 280 \\
\hline OPC-0.5 & 0.5 & 0 & 700 & 0 & 350 \\
D2-0.5 & 0.5 & 2 & 686 & 14 & 350 \\
D6-0.5 & 0.5 & 6 & 658 & 42 & 350 \\
D10-0.5 & 0.5 & 10 & 630 & 70 & 350 \\
\hline OPC-0.6 & 0.6 & 0 & 700 & 0 & 420 \\
D2-0.6 & 0.6 & 2 & 686 & 14 & 420 \\
D6-0.6 & 0.6 & 6 & 658 & 42 & 420 \\
D10-0.6 & 0.6 & 10 & 630 & 70 & 420 \\
\hline
\end{tabular}

1.10. Rheological and mini cone slump test

The rheometer (Model: Anton Paar MCR 502) with a co-cylindrical cup-and-bob configuration (gap size of $1.13 \mathrm{~mm}$ ) was used to measure the rheological properties of all pastes. During the tests, the cement pastes were first pre-sheared at shear rate of $600 \mathrm{~s}^{-1}$ for 10 seconds to minimize the effects of shear history so that all the samples start from the same reference point. The paste was then let rest for 3 minutes. After that, samples were sheared based on a descending flow curve at 6 different shear rates $(300,250,200,150$, 100 , and $50 \mathrm{~s}^{-1}$ ). Samples were maintained at each shear rate for 10 seconds and the corresponding shear stresses were measured. Bingham model was applied to calculate the viscosity and the yield stress.

The mini-cone slump tests were also conducted for all the paste samples to measure the flow diameter. The average of the flow diameter in two perpendicular directions was 
used to represent the flow diameter of the corresponding cement paste. All Rheological and mini-cone slump tests were conducted 13 minutes after initial mixing.

Viscosity and yield stress of all different cement pastes are calculated based on the obtained flow curves using Bingham model (Banfill, 2003). Figure 13 andFigure 14 show the calculated viscosities and yield stresses, respectively. It can be seen clearly that by increasing DE replacement level, viscosity is increased for all the w/b ratios. Rheological properties of cement pastes is highly related to its inter-particle and non-contact surface forces (van der Waals, double layer) (Banfill, 1990; Lootens, Hébraud, Lécolier, \& Van Damme, 2004). DE particles, due to their high Blaine surface and porous structure, tend to absorb free water available in the paste. Although, this water will be released back to cement matrix in later ages, in early age, it causes reduction in water content. It should also be noticed that the specific gravity of DE is lighter than cement. When cement is replaced by DE with the same mass, the solid volume fraction increases. The increased solid volume fraction together with the porous texture of DE result in higher inter-particles interactions and consequently higher viscosity.

Figure 14 depicts changes in yield stress by different replacement levels of DE. It shows that for 0.5 and $0.6 \mathrm{w} / \mathrm{b}$ ratios, increasing DE replacement level in cement pastes, constantly leads to a drop in yield stress. However, for $0.4 \mathrm{w} / \mathrm{b}$ ratio, increasing replacement level at first doesn't change yield stress significantly (it drops slightly from $25.75 \mathrm{~Pa}$ for OPC-0.4 to 25.42 Pa for D2-0.4). While thereafter, by keep increasing DE content from $2 \%$ to $6 \%$, yield stress increases considerably (from $25.42 \mathrm{~Pa}$ for D2-0.4 to $30.32 \mathrm{~Pa}$ for D6-0.4). 


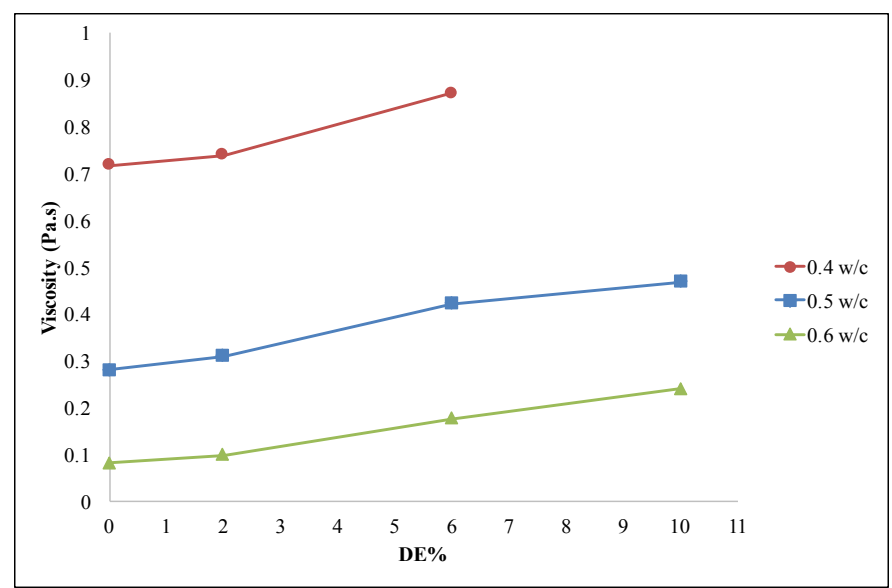

Figure 13: Viscosity of cement pastes against DE replacement levels

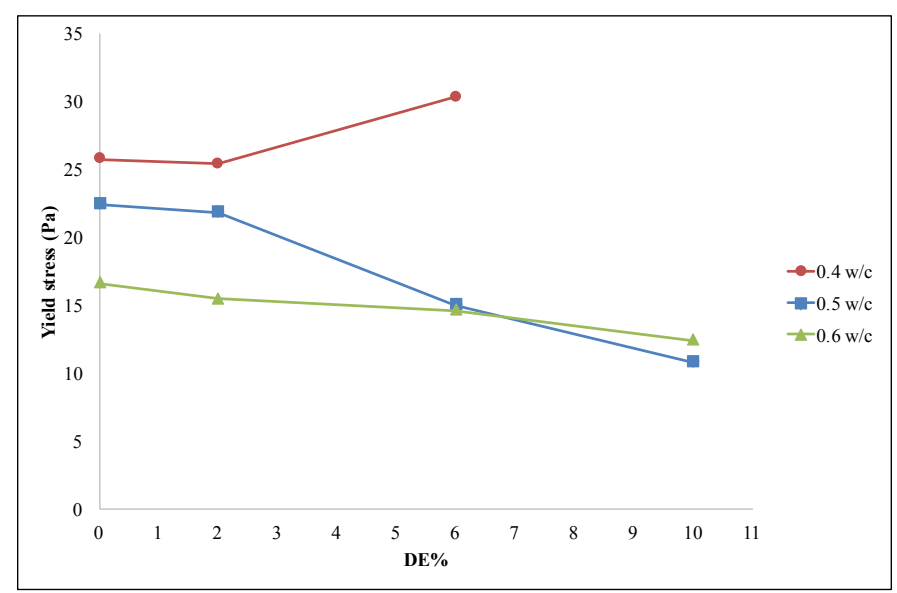

Figure 14: Yield stress of cement pastes against DE replacement levels

To explain these changes in yield stresses of cement pastes with different $\mathrm{w} / \mathrm{b}$ ratios and different DE replacement levels, the change in their shear behavior should be considered. Shear thinning and shear thickening are typical for non-Newtonian flow fluids, in which viscosity non-linearly decreases or increases by increase in shear rate (He, Gong, Xuan, Jiang, \& Chen, 2015; Yahia, 2014). Shear-thinning is generally attributed to shearinduced de-flocculation while shear-thickening is generally attributed to repulsive interactions between both colloidal and non-colloidal particles in the case of suspensions (Bouras, Kaci, \& Chaouche, 2012). 


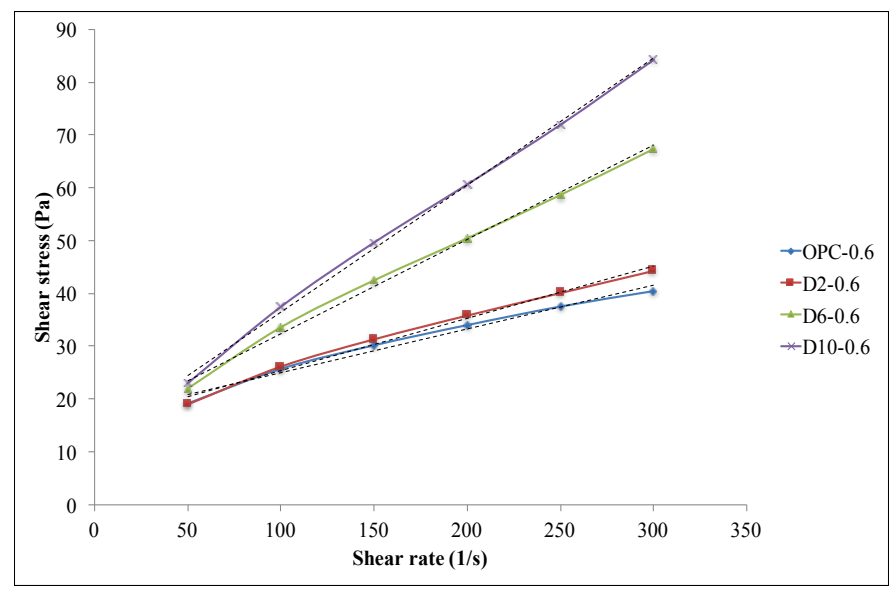

Figure 15: Flow curve of cement pastes with $0.6 \mathrm{w} / \mathrm{b}$ ratio

Figure 15 and Figure 16 show flow curves of cement pastes with water to binder ratio of 0.6 and 0.4 , respectively $(0.5 \mathrm{w} / \mathrm{b}$ ratios had very similar flow curves as of 0.6$)$. It can be seen that the control cement paste (OPC-0.6) with no additive shows a clear shear thinning behavior all along its flow curve while OPC-0.4 first shows shear thinning at low shear rates and then shear thickening behavior at higher shear rates. This is in agreement with finding of previous research which showed cement pastes with lower w/c ratios show more shear thickening behavior than pastes with higher w/c ratios at high shear rates (Yahia, 2011). Also, one can easily see that by increasing DE replacement level, flow curves from both w/b ratios $(0.6$ and 0.4$)$ show more shear thickening behavior at higher shear rates. This can be explained by porous structure and high Blaine surface of DE particles. According to He et al. (He et al., 2015), the surface characteristics of dispersed particles significantly affects the rheological properties of the shear thickening fluids (STFs). They found that by dispersing porous particles (like porous silica fume) in polar liquids, the high specific surface areas and roughness surface nature of the porous particles may influence the interfacial interaction between particles and dispersing medium, and improve shear thickening behavior. From Figure 15, it can also be seen that using DE to partially 
replace cement not only change the flow behavior from shear thinning to shear thickening at high shear rate, but also the higher the DE dosage, the more obvious this transition would be.

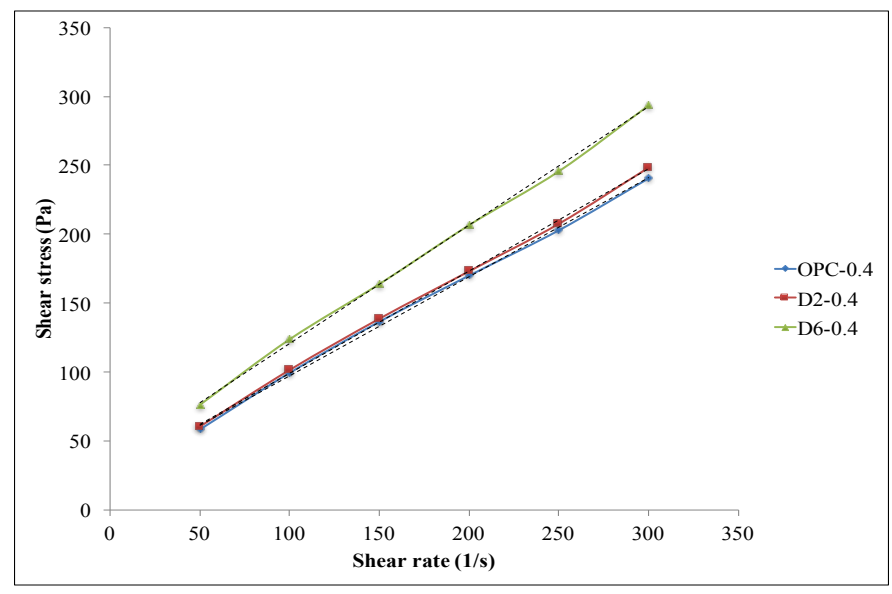

Figure 16: Flow curve of cement pastes with $0.4 \mathrm{w} / \mathrm{b}$ ratio

Flow diameters of cement pastes with different $\mathrm{w} / \mathrm{c}$ ratios and DE replacement levels are shown in Figure 17. The results show a consistent decrease in flow diameter by increasing DE content. This can be explained by the high Blaine surface, porous structure and water absorbent nature of DE particles, which leads to lower water content in cement pastes and therefore less flowability (flow diameter). According to previous studies (Ferraris, Obla, \& Hill, 2001; Wallevik, 2006) there is a correlation between minislump flow diameter and yield stress. A lower yield stress corresponds to a higher spread in the minislump (Ferraris et al., 2001). However, this research shows the slump flow can be related to both viscosity and yield stress. For the pastes with DE replacement, the viscosity may dominate the flow diameter. 


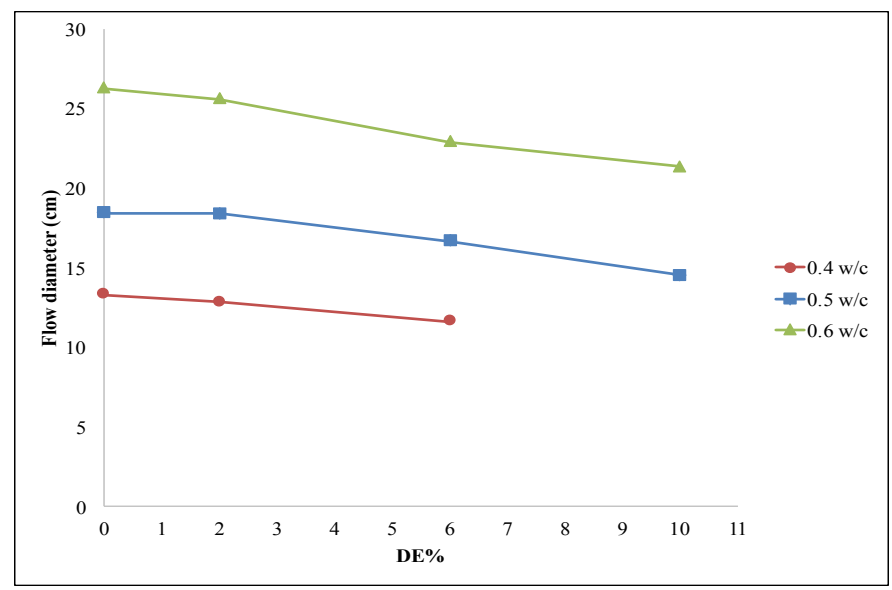

Figure 17: Flow diameter of cement pastes against DE replacement levels

It should be pointed out that, due to its high water demand, paste D10-0.4 exhibited very poor workability, (Agullo, Toralles-Carbonari, Gettu, \& Aguado, 1999; Degirmenci \& Yilmaz, 2009; Fragoulis et al., 2005; Miller et al., 2010; Stamatakis et al., 2003; Y1lmaz \& Ediz, 2008), and implementing rheological and mini-cone slump tests were not feasible for this cement paste.

\subsection{Bleeding test}

To conduct bleeding test, the pastes were poured to within $0.6 \mathrm{~cm}(1 / 4 \mathrm{inch})$ from the top of the $5 \mathrm{~cm}(2 \mathrm{inch})$ by $10 \mathrm{~cm}$ (4 inch) cylinders and then weighed. The containers were capped after weighing to prevent moisture loss. After 30 minutes elapsed, the cylinders were tilted at $30^{\circ}$ from vertical and bleed water was removed with a transfer pipette. Caution was taken to prevent removing any of the paste with the bleed water. Once no more visible bleed water remained on the surface, the cylinders were weighed and capped with a plastic lid. This process was repeated every 30 min until no further bleed water was observed. The reported value of weight loss and weight loss rate is the averages of the three specimens (Sun \& Young, 2014). 


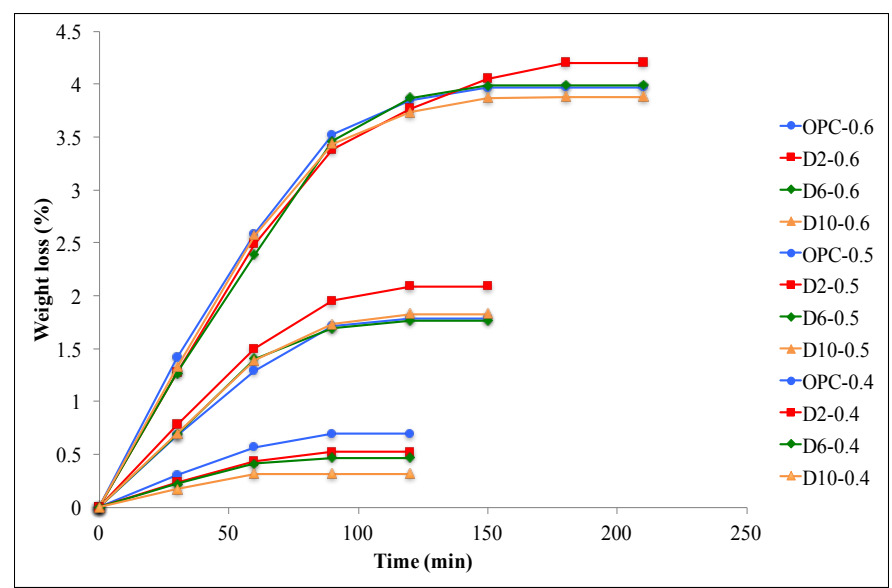

Figure 18: Weight loss of cement pastes against time

Figure 18 plots the changes of weight loss due to bleeding against time. As it is clear from this figure, for pastes with $0.4 \mathrm{w} / \mathrm{b}$ ratio, by increasing the replacement level of $\mathrm{DE}$ in cement paste, the weight loss is decreasing for all time intervals. However, for 0.5 and 0.6 pastes, no clear pattern is observed. In the case of $0.4 \mathrm{w} / \mathrm{b}$ ratio, the bleeding water could be clearly identified and removed from the top of the specimen. While in 0.5 and 0.6 $\mathrm{w} / \mathrm{b}$, it is hard to differentiate bleeding water and diluted paste layer. Some cement particles may be removed, together with the water when the pipette is used. Therefore, the trend line plotted with higher w/b ratios, probably contain higher measurement errors. Hence, the focus of the data analysis on bleeding will be on the $0.4 \mathrm{w} / \mathrm{b}$ pastes.

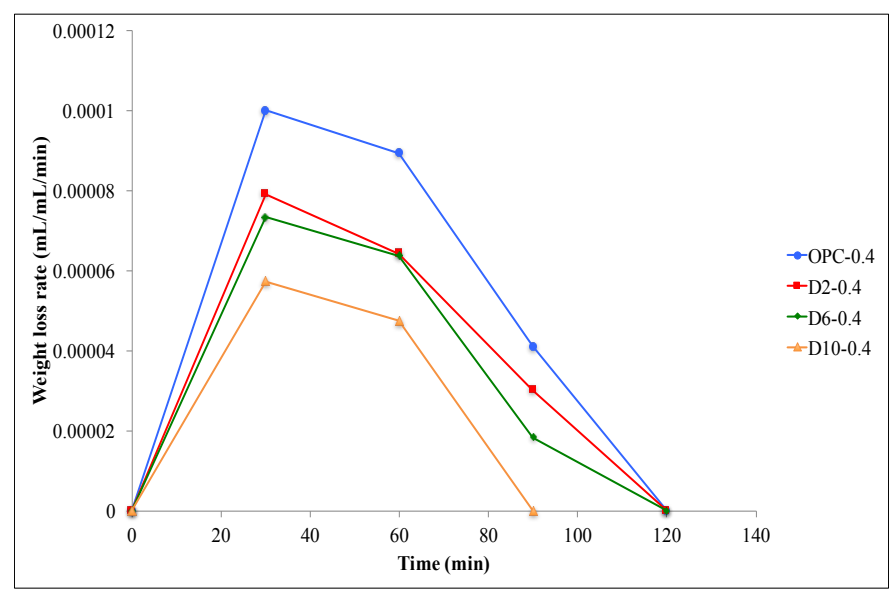

Figure 19: Weight loss rate of cement pastes against time $(0.4 \mathrm{w} / \mathrm{b}$ ratio $)$ 
Figure 19 plots the changes in bleeding rates for all the pastes with $\mathrm{w} / \mathrm{b}$ ratio of 0.4 . One can see that for a given age, e.g. 30 minutes, the control specimen has the highest bleeding rate, and the rate decreases accordingly with the increase of DE replacement. This indicates that adding DE to cement paste can delay and reduce bleeding effectively. From the figure, it can also be noticed that bleeding for the D10-0.4 paste stopped earlier than any other pastes. This is another indication of the minimizing the bleeding effect by using DE.

\subsection{Setting time}

The initial and final setting time of all cement pastes were determined by the Vicat needle tests, which were carried out according to ASTM C191 (ASTM, 2008). When the needle penetrated into the cement paste for $25 \mathrm{~mm}$, initial set was achieved. Final setting was attained when there was no mark of the specimen surface with a complete circular impression (Liu, Sun, \& Qi, 2014).

Initial and final setting time of all cement pastes with different water to binder ratios and DE replacement levels are provided in Table 6 . It is observed that for all the $\mathrm{w} / \mathrm{b}$ ratios that studied, increasing DE replacement levels causes decreases in both initial and final setting times, it also shortens the time difference between initial and final settings for all the 0.4 and 0.5 pastes. Also, amongst cement pastes with the same DE content those with higher w/c ratio have longer initial and final setting as expected. In addition, it can be seen that in cement pastes with lower $\mathrm{w} / \mathrm{b}$ ratios, the rate of reduction in setting time (final setting) by increasing DE content is much higher than those with higher w/b ratios. For ex- 
ample, it took 110 minutes less for D10-0.4 to reach final set compared to OPC-0.4, however, the final setting time has only been shortened by 35 minutes for 0.6 pastes when $10 \%$ of the cement is replaced by DE.

Table 6: Initial and final setting of cement pastes with different w/c ratios and DE replacement levels

\begin{tabular}{ccc}
\hline Cement paste & initial set $(\min )$ & final set $(\min )$ \\
\hline OPC- 0.4 & 226 & 350 \\
D2-0.4 & 178 & 310 \\
D6-0.4 & 166 & 255 \\
D10-0.4 & 141 & 240 \\
\hline OPC-0.5 & 268 & 450 \\
D2-0.5 & 246 & 390 \\
D6-0.5 & 234 & 375 \\
D10-0.5 & 229 & 360 \\
\hline OPC-0.6 & 340 & 570 \\
D2-0.6 & 328 & 565 \\
D6-0.6 & 300 & 555 \\
D10-0.6 & 275 & 535 \\
\hline
\end{tabular}

Replacing cement by DE reduces the concentration of cement particles in cement paste (i.e. reduces cement content) and is expected to prolong setting time. But on the other hand, DE particles due to their porous structure and high Blaine surface can absorb some of the free water in the paste that lowers the free water content and improve diffusion of water in cement matrix which shortens the time for water to reach the non-hydrated cement.

\subsection{Isothermal calorimetry}

The heat of hydration of the all cement pastes are measured by the isothermal calorimetric test as defined by ASTM C1702 (ASTM, 2009). TAM Air, a commercial calorimeter, was adopted in this study. It is an eight-channel isothermal heat conduction calorimeter with the operating temperature range between 5 and $60^{\circ} \mathrm{C}$. Before testing, the 
equipment was carefully calibrated based on the calibration procedures specified by the manufacturer. The energy change during hydration was collected and registered by an automated data acquisition program. The energy value was calculated based on the unit weight of cementitious materials mass.

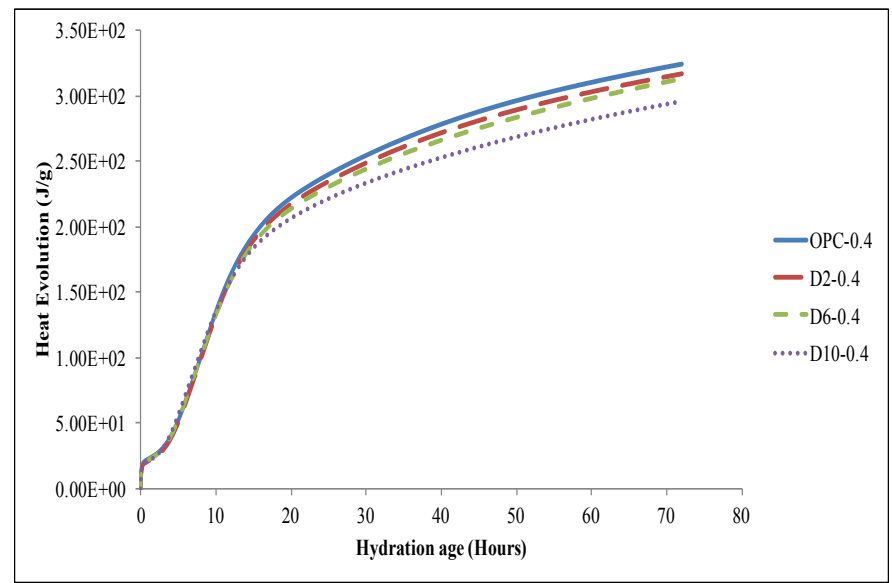

Figure 20: Heat evolution of cement pastes with $0.4 \mathrm{w} / \mathrm{b}$ ratio

For all water to binder ratios increase in DE content reduced heat evolution of cement pastes on first 70 hours. As an example, heat evolution of pastes with $0.4 \mathrm{w} / \mathrm{b}$ ratios and different DE replacement levels are shown in Figure 20.

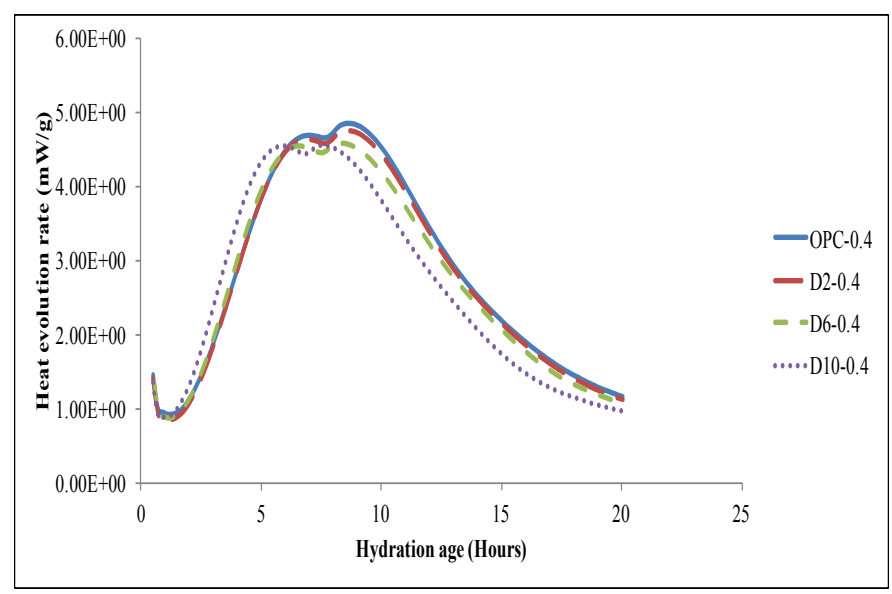

Figure 21: Heat evolution rate of cement pastes with $0.4 \mathrm{w} / \mathrm{b}$ ratio

Figure 21 to Figure 23 show the rate of heat evolution of all the cement pastes against time. It should be pointed out that these figures are cut and enlarged from hydration 
age 0.5 to 20 hours for better illustration of changes in peaks of heat evolution rate. The first peak in the figure is mainly associated with hydration of $\mathrm{C}_{3} \mathrm{~S}$ in cement paste and the second peak is mostly attributed to the hydration of $\mathrm{C}_{3} \mathrm{~A}$ (Mostafa \& Brown, 2005; Pang, Bentz, Meyer, Funkhouser, \& Darbe, 2013; Rahhal \& Talero, 2009). It is clear from these figures that increase in $\mathrm{DE}$ content results in a drop in heat evolution rate of both peaks for all $\mathrm{w} / \mathrm{b}$ ratios. As mentioned in previous section, this is simply due to the decrease in clinker phases (cement content), which is caused by replacing of cement with DE.

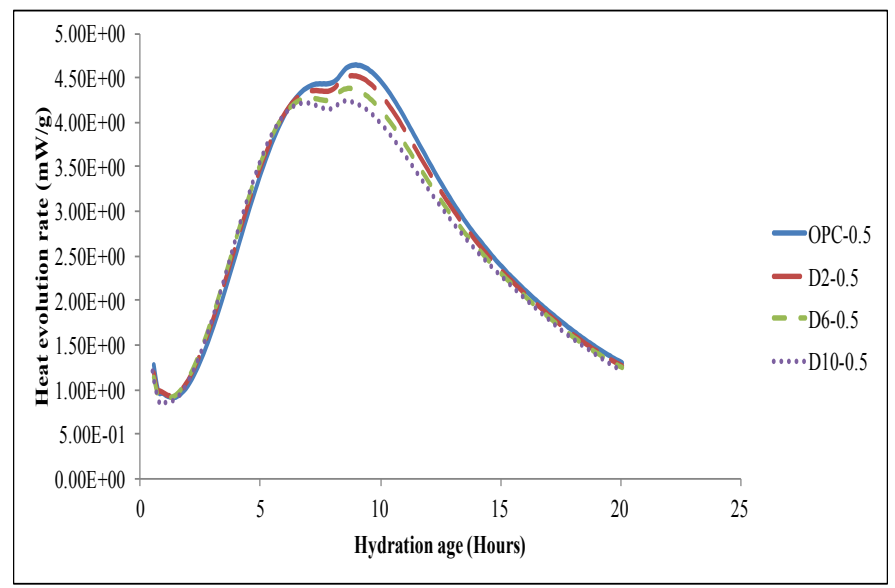

Figure 22: Heat evolution rate of cement pastes with $0.5 \mathrm{w} / \mathrm{b}$ ratio

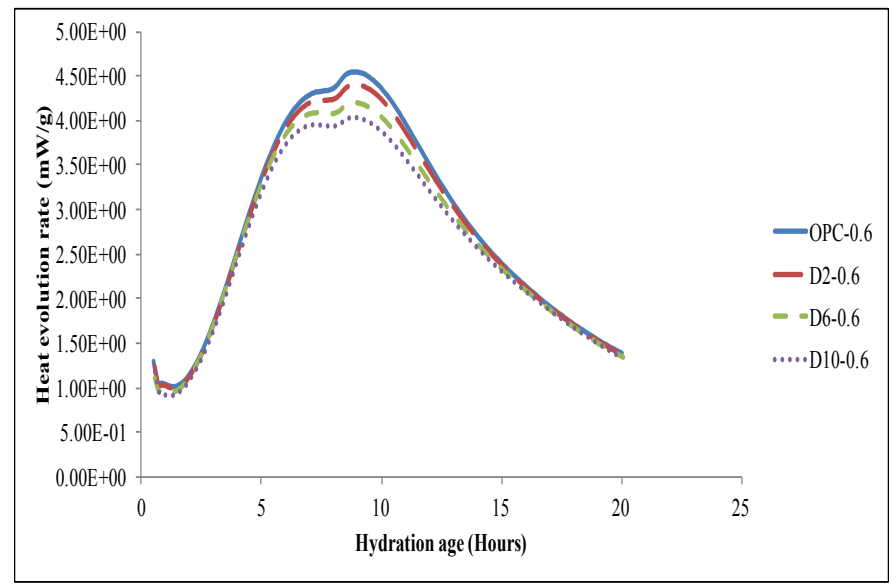

Figure 23: Heat evolution rate of cement pastes with $0.6 \mathrm{w} / \mathrm{b}$ ratio 
In addition, for all water to binder ratios, higher DE content led to shifts (to the left) for both peaks. The higher the DE content the larger the shift is (Table 7). Also, the shift with increase in DE content is observed to be more obvious for cement pastes with lower $\mathrm{w} / \mathrm{b}$ ratios.

Table 7: Hydration age of first and second peaks of heat evolution rate for all cement pastes

\begin{tabular}{ccc}
\hline Cement paste & first peak $(\mathrm{h})$ & second peak $(\mathrm{h})$ \\
\hline OPC-0.4 & $\mathbf{7 . 0 2 6 7 6}$ & $\mathbf{8 . 6 9 3 4 3}$ \\
D2-0.4 & -0.08975 & -0.08976 \\
D6-0.4 & -0.43464 & -0.35131 \\
D10-0.4 & -1.09506 & -1.09506 \\
\hline OPC-0.5 & $\mathbf{7 . 8 8 2 6 1}$ & $\mathbf{8 . 9 6 5 9 4}$ \\
D2-0.5 & -0.48359 & -0.15025 \\
D6-0.5 & -0.81736 & -0.23402 \\
D10-0.5 & -0.96151 & -0.29484 \\
\hline OPC-0.6 & $\mathbf{7 . 9 3 6 2 8}$ & $\mathbf{9 . 0 1 9 6 1}$ \\
D2-0.6 & -0.23428 & -0.15094 \\
D6-0.6 & -0.52509 & -0.19175 \\
D10-0.6 & -0.72741 & -0.22741 \\
\hline
\end{tabular}

These results fully support discussion in previous section and research findings from the setting time test. The acceleration of peak appearance can be attributed to the absorption of free water to DE particles that cause the increase of solution of cement clinkers (like $\left.\mathrm{C}_{3} \mathrm{~A}\right)$ in water. This leads to higher solubility of ions such as $\mathrm{Ca}^{2+}$ (i.e. higher zeta potential). It in turn accelerates formation of hydration products and shortens setting times (Nägele, 1985; Plank \& Hirsch, 2007; Yılmaz \& Ediz, 2008). Kastis et al. (Kastis et al., 2006) pointed out that the pozzolanic nature of diatomite can form higher amounts of hydration products in paste. Based on the findings of Rahhal and Talero (Rahhal \& Talero, 2009), the hydration reactions may be stimulated by the positive and negative electrostatic 
charge acquired by the particles of pozzolans during mixing, and subsequently, by the zeta potential specially originated as Portland cement hydration progresses. Therefore, DE particles could play the role as nucleation sites (like seed crystal) for calcium hydroxide crystals to precipitate with accelerated setting (Rahhal \& Talero, 2009). Putting Figure 21 to Figure 23 together with Table 6, one can see that the earlier the peaks appear, the quicker the paste would set.

\subsection{Conclusions}

Findings of this paper show that using Diatomaceous Earth (DE) as a partial replacement for cement has significant influence on fresh properties of cement pastes. The porous shape and high Blaine surface of DE particles makes this natural pozzolanic additive of cement pastes to be highly water absorbent. DE can impact fresh properties of cement pastes as follow:

- Increase in DE content of cement pastes increased the apparent viscosity of cement pastes with w/b ratios of $0.4,0.5$ and 0.6 . It can be due to higher solid volume fraction of cement pastes with higher DE content which leads to higher inter-particle interactions.

- Increase in DE content changed the shear behavior of cement pastes from shear thinning to shear thickening behavior. This change in shear behavior is clearer in cement pastes with 0.5 and $0.6 \mathrm{w} / \mathrm{b}$ ratios.

- Increase in DE content decreased flow diameter of cement pastes at all water to binder ratios. 
- Increase in DE content of cement pastes reduced bleeding rate of cement pastes with $0.4 \mathrm{w} / \mathrm{b}$ ratio.

- Increase in DE content shortened both initial and final setting time of cement pastes at all $\mathrm{w} / \mathrm{b}$ ratios. DE particles improve water diffusion, increase zeta potential and accelerate formation of hydration products which lead to shorter setting times.

- Increase in DE content lowers the hydration heat of cement pastes at all water to binder ratios due to lower cement content of cement pastes.

- Results from isothermal calorimetry show that increase in replacement level of cement with DE hasten the hydration process in cement pastes with all water to cement ratios which is in good agreement with results from setting time test. 


\section{CHAPTER 4}

\section{THIXOTROPIC PROTOCOLS}

\subsection{Introduction}

As mentioned earlier in Chapter 2, common methods of measuring thixotropy are incapable of providing a reliable and comprehensive conception of thixotropic behavior. The main defect with these methods is their failure to distinguish and measure the two different aspects of thixotropy i.e. breakdown and buildup. They either combine these two aspects or only consider one. For cementitious materials, structural breakdown and buildup have different applications. As one represent material behaviors during construction and the other represents other properties after construction. A comprehensive understanding of thixotropic behavior of materials requires a distinctive understanding of both aspects.

In this chapter, a series of protocols are proposed to quantitatively measure thixotropic behavior of cementitious pastes. These protocols are intended to give a more comprehensive and reliable understanding of thixotropic behavior. For that purpose, four different thixotropic protocols are proposed. The first two protocols address the breakdown aspect of thixotropy and the next two protocols address its buildup. The quantitative results from these protocols not only make it possible to analyze breakdown and buildup, separately; but also, provide the opportunity to study the correlation between these two aspects of thixotropy. 
The capability of the proposed protocols in determining thixotropic behavior of cementitious pastes have also been examined. For that purpose, using proposed protocols, a series of tests on different cementitious pastes are conducted. Cementitious pastes with different water content and mix proportions are selected. Raw materials, mix proportions and mixing procedure are the same as those in Chapter 3, see Table 3-Table 5. To simulate on-site conditions, the influence factors such as temperature and pressure are also considered. Finally, the test results from the proposed protocols are used to study and discuss the impact of each factor (water content, mix proportion, temperature and pressure) on thixotropic behavior of cementitious pastes, i.e. breakdown, buildup and the correlation of these two aspects.

\subsection{Proposed thixotropic protocols}

\subsubsection{Protocol 1 (easiness of breaking down to steady state)}

This protocol is developed to measure the easiness of breaking the cement pastes' structure down to a steady state (equilibrium condition) under a high shear rate. This steady state is considered as the most deflocculated state that cement paste can practically reach under a certain high shear rate. At this state, the rate of flocculation and de-flocculation are assumed to be in balance.

During the test, when the pastes were placed in the rheometer, all samples were firstly pre-sheared at a high speed of $300 \mathrm{~s}^{-1}$ for $10 \mathrm{~s}$ and then followed by 3 min rest to eliminate discrepancies caused by the shear history in mixing so that all the samples would have the same initial starting point for the following measurement. Then, a constant high shear rate of $300 \mathrm{~s}^{-1}$ was applied on the samples for $20 \mathrm{~min}$. A schematic diagram of protocol 1 procedure is shown in Figure 24. During this $20 \mathrm{~min}$, the alternation of shear stress 
was recorded and plotted against time. Therefore, minimum time to reach to equilibrium condition $(\Delta \mathrm{T})$, drop in shear stress $(\Delta \tau)$ and break-down energy (BDE) can be calculated as shown in Figure 25. Among which, $\Delta \mathrm{T}$ indicates the quickness/easiness to breakdown a material. Similarly, a smaller value of BDE indicates less energy consumption to reach equilibrium condition, therefore, a material with less BDE can be consider a material with more easiness of breaking down.

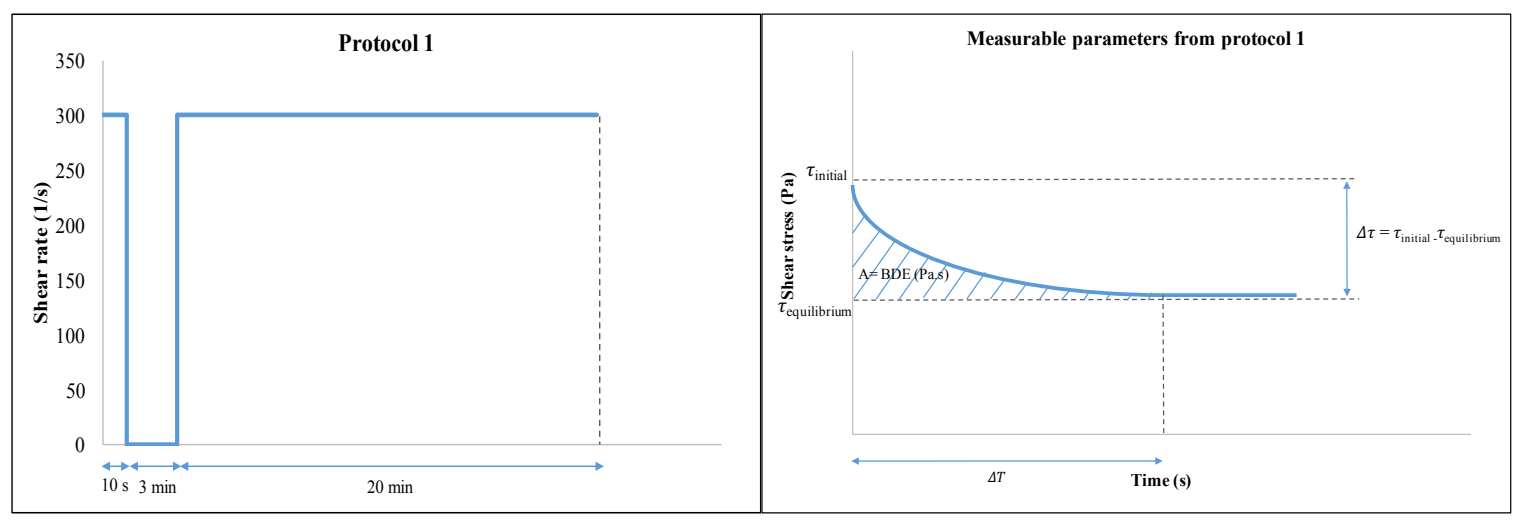

Figure 24: Protocol 1 procedures

Figure 25: Measurable parameters from protocol 1

\subsubsection{Protocol 2 (easiness of starting the flow)}

This protocol is intended to measure the easiness to initiate flow in a cementitious paste. Static yield stress is an important indication of how hard need to push to initiate the flow of a liquid. A higher value of static yield stress indicates a harsh or difficult start to mix or pump the material. Therefore, it provides a quantitative measurement of the breakdown aspect of thixotropy. For this purpose, after usual $10 \mathrm{~s}$ of pre-shear at $300 \mathrm{~s}^{-1}$, and 3 min rest, a simple shear stress ramp from 0 to $50 \mathrm{~Pa}$ (assuming $50 \mathrm{~Pa}$ is big enough to include the static yield stress of the measured paste) in rate of $0.1 \mathrm{pa} / \mathrm{s}$ is applied on samples. During the ramp, the alternation of shear rate is recorded and plotted against shear 
stress. Thus, the shear stress at which samples start to yield, static yield stress, are measured. A schematic diagram of protocol 2 procedures and an example of obtained data are shown in Figure 26 and Figure 27 respectively.

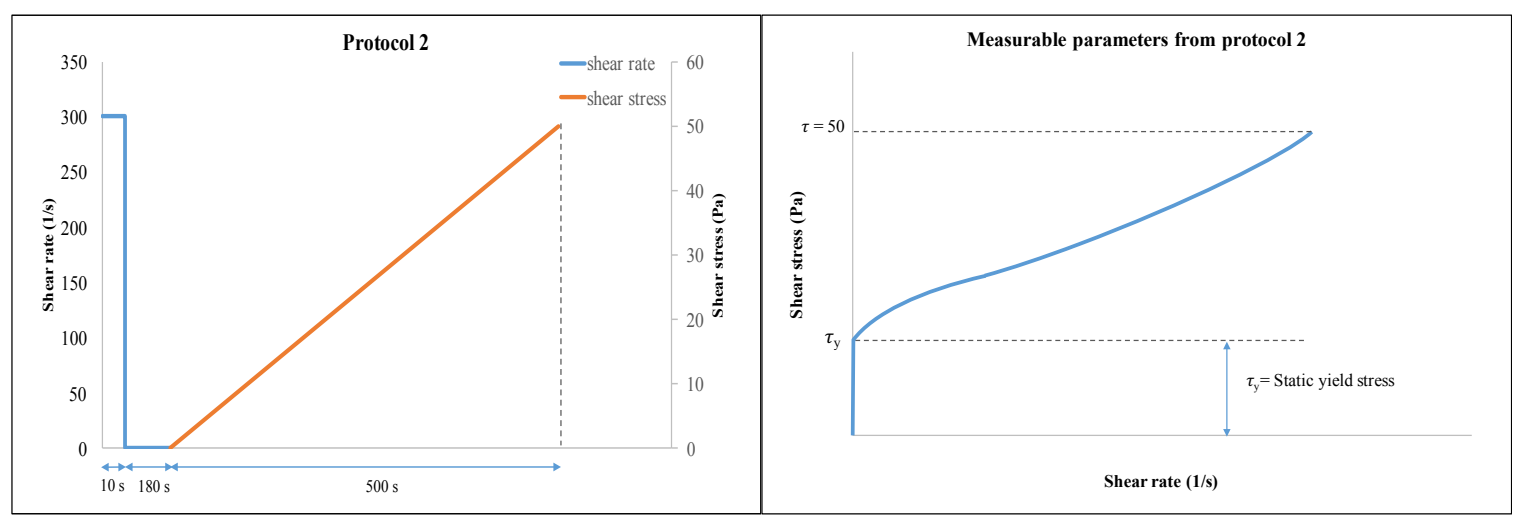

Figure 26: Protocol 2 procedures

Figure 27: Measurable parameters from protocol 2

\subsubsection{Protocol 3 (measuring buildup ratio by monitoring shear stress buildup)}

Protocol 3 is intended to calculate the buildup ratio of sample before and after its structural breakdown. In this protocol, after pre-shearing, a very small shear rate of 0.0001 $\mathrm{s}^{-1}$ is applied on the sample for $3 \mathrm{~min}$ and the buildup of shear stress is recorded. This shear rate is purposely chosen to be within its linear viscoelastic region (LVER) to prevent damaging the micro-structure of cement paste. Then, a constant high shear rate of $300 \mathrm{~s}^{-1}$ is applied for $8 \mathrm{~min}$ to break the structure down to a steady state (or the equilibrium). After structural breakdown, the low shear rate of $0.0001 \mathrm{~s}^{-1}$ is applied again to monitor the rebuilding of structure. The measurement continues till sample reaches its initial state just before the breakdown step, see Figure 28. By plotting the measured shear stress against time, buildup ratio $\left(\mathrm{B}_{\mathrm{R}}\right)$ and the time to rebuild initial state $\left(\Delta \mathrm{T}_{\text {buildup }}\right)$ are calculated as shown in Figure 29. A higher $\Delta \mathrm{T}_{\text {buildup }}$ value indicates a lower buildup speed and similarly, a higher $B_{R}$ value indicate a more thixotropic behavior in terms of structural rebuilding. 


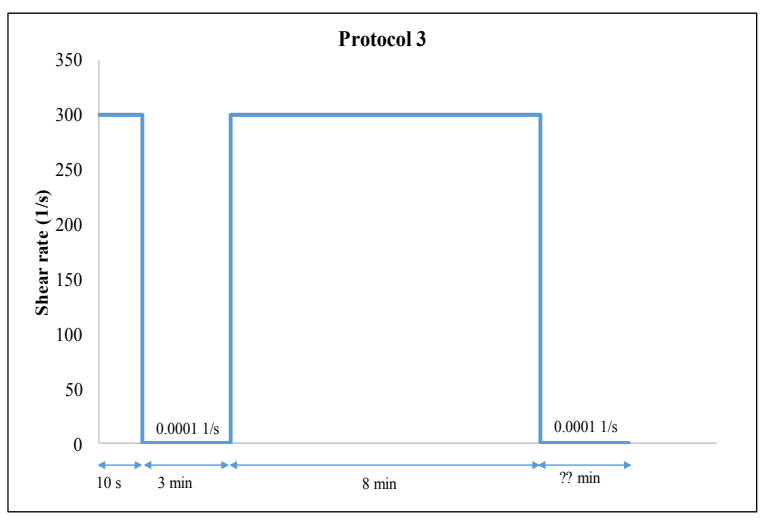

Figure 28: Protocol 3 procedures

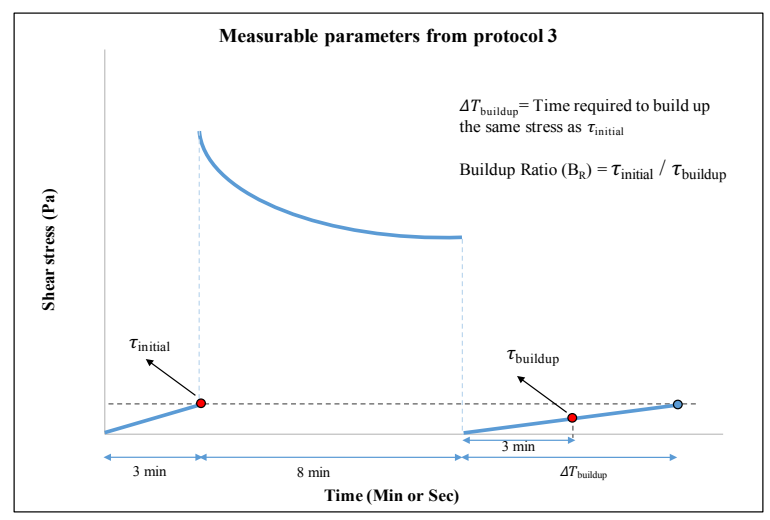

Figure 29: Measurable parameters from protocol 3

\subsubsection{Protocol 4 (measuring buildup ratio by monitoring static yield stress)}

This protocol is designed, based on protocol 2 and 3 , to measure the rebuilding ratio of samples before and after breaking down of their structure. In protocol 4, instead of monitoring shear stress buildup, the static yield stress before and after structural breakdown are monitored. As shown in Figure 30, after usual pre-shearing and 3 min rest, a shear stress ramp with the rate of $0.1 \mathrm{~Pa} / \mathrm{s}$ for 4 minutes is applied. This is followed by a constant high shear rate of $300 \mathrm{~s}^{-1}$ shear rate for 8 min like protocol 3 to achieve structural breakdown and then another shear stress ramp with the same rate of $0.1 \mathrm{~Pa} / \mathrm{s}$ and $4 \mathrm{~min}$ is applied again. Thus, by plotting shear stress against shear rate two static yield stresses, one before structure breakdown and one after structure breakdown, are obtained. The ratio $\left(\tau_{\mathrm{y} 2} / \tau_{\mathrm{y} 1}\right)$ have been used to calculate buildup ratio (see Figure 31). 


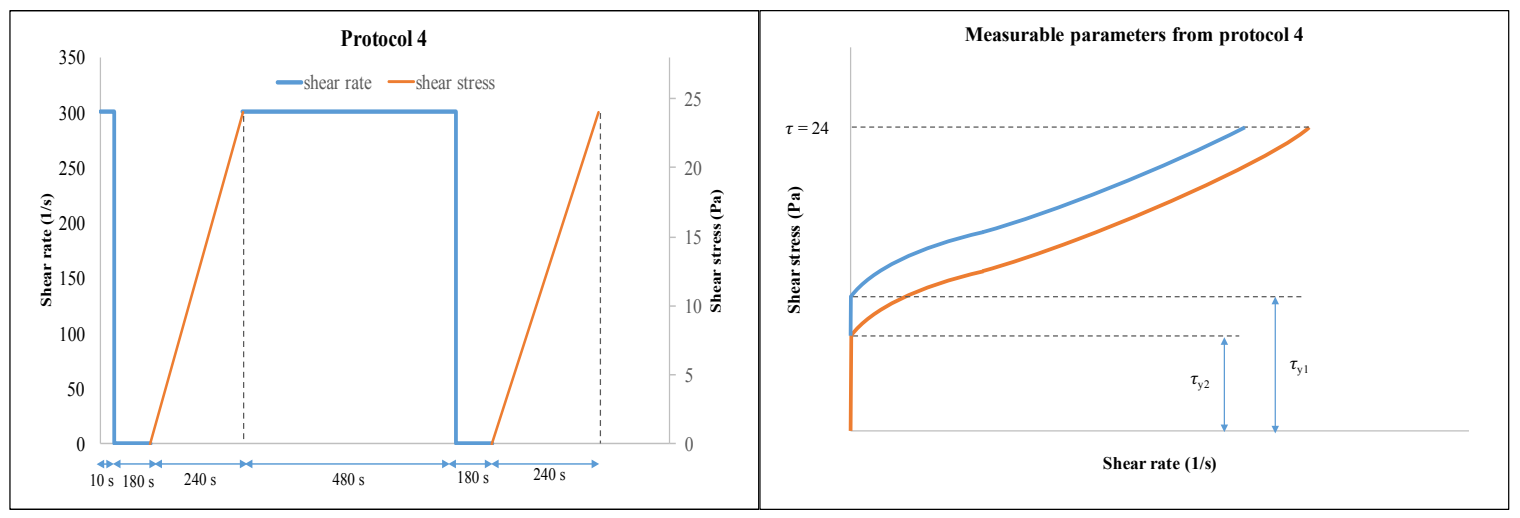

Figure 30: Protocol 4 procedures

Figure 31: Measurable parameters from protocol 4

\subsection{Results and discussion}

\subsubsection{Structural Breakdown}

Results from protocol 1 are shown in Figure 32 to Figure 37. Figure 32 and Figure 33 show breakdown energy of cementitious pastes against their DE replacement level and w/b ratio, respectively. It can be seen that by increasing DE content, breakdown energy of cement pastes with almost all $\mathrm{w} / \mathrm{b}$ ratios was decreased. In other words, under a constant high shear rate, increasing DE content can help to reduce the needed energy to reach the steady state of the flow. So, it can be concluded that cement pastes with higher DE content are easier to break down. Also, from Figure 33, by increase in w/b ratio at any DE replacement level, breakdown energy was decreased. This is reasonable because the sample with a higher $\mathrm{w} / \mathrm{b}$ ratio has a loose microstructure with less solid particles to breakdown. Figure 34 and Figure 35, plot the drop of shear stress to reach the steady state against DE content and $w / b$ ratio, respectively. Cementitious pastes with higher DE content in general show slightly higher stress drop than those with lower DE contents. Also, from Figure 35, cement pasts with higher $w / b$ ratio have lower drop in shear stress. Again, this corresponds to the solid concentration in cement pastes. Figure 36 and Figure 37, show time to reach to steady 
state against DE content and w/b ratio, respectively. In Figure 36, $\Delta \mathrm{T}$ (time to reach steady state) for a given $w / b$ ratio was decreased by increase in $\mathrm{DE}$ content. It indicates that a paste with a higher DE content is probably easier to breakdown. This can be attribute to the negative charges on the surface of DE particles that repel to help the separation of solid particles. Also, from Figure 37, almost for all DE replacement levels, increase in water content leads to longer time to reach to steady state. However, for $6 \%$ replacement level higher water content leads to slightly shorter $\Delta \mathrm{T}$. This indicate that $6 \%$ of $\mathrm{DE}$ content is probably a threshold that convert the breakdown aspect of thixotropic behaviors of the paste.

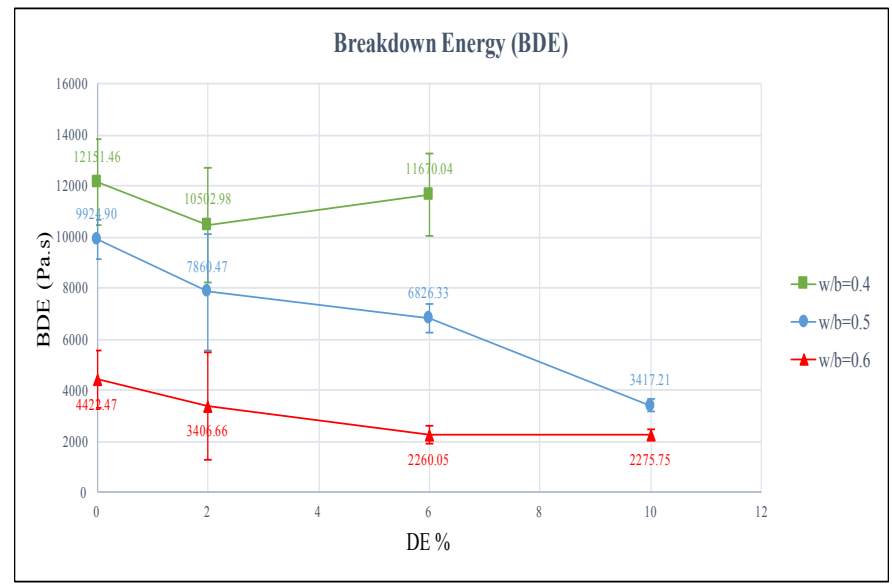

Figure 32: Breakdown energy against DE replacement level from protocol 1

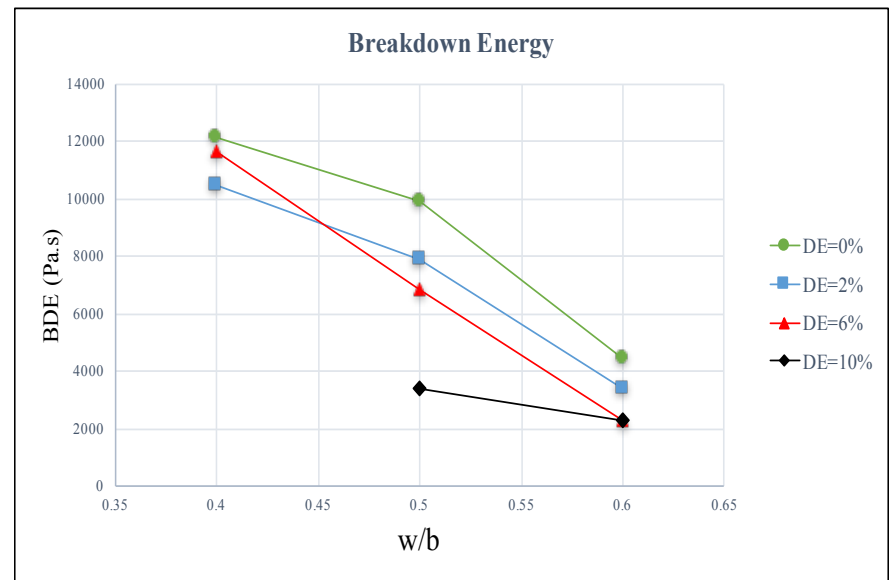


Figure 33: Breakdown energy against $w / b$ ratio from protocol 1

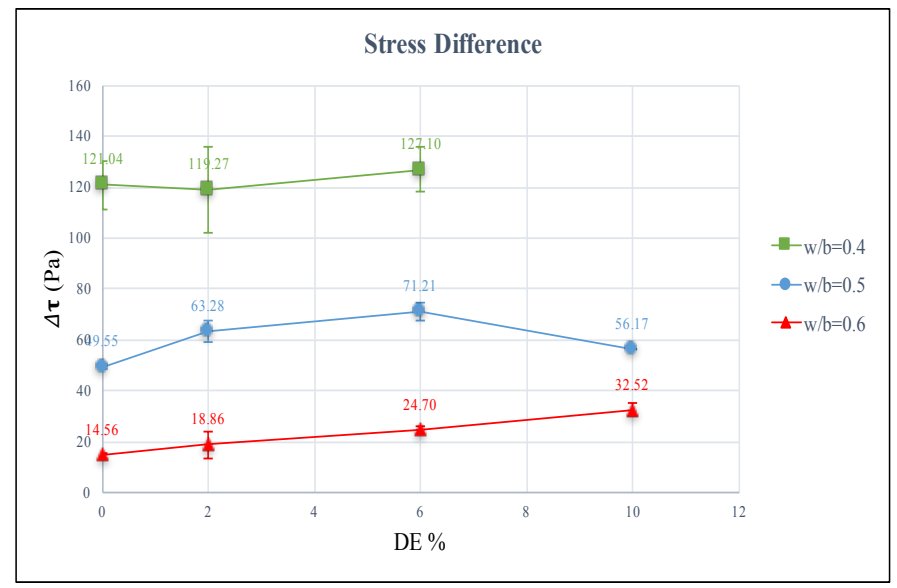

Figure 34: Drop in shear stress against DE replacement level for protocol 1

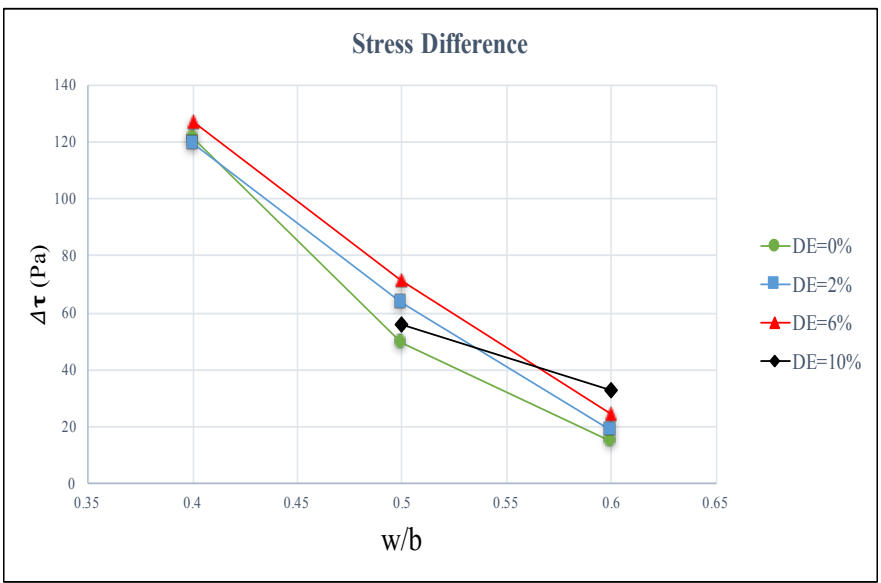

Figure 35: Drop in shear stress against $w / b$ ratio for protocol 1

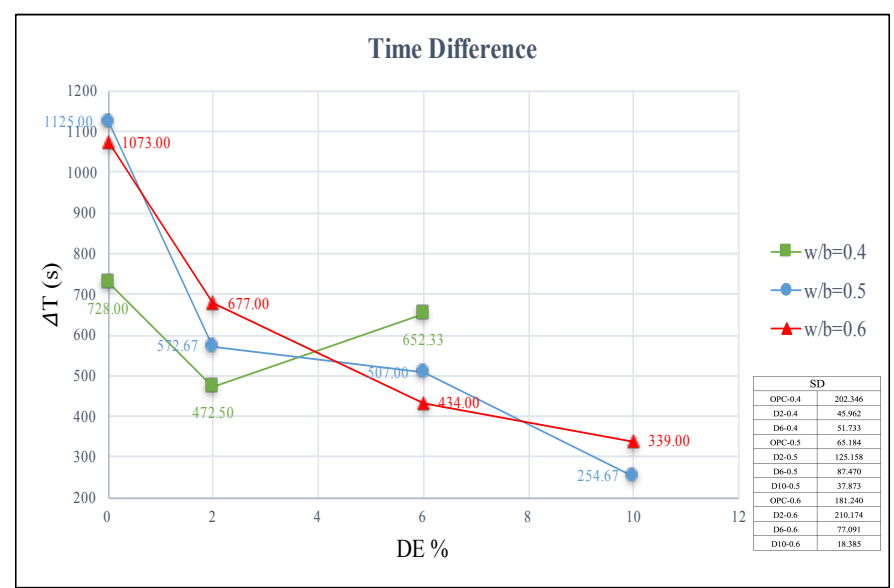

Figure 36: Time to reach to steady state against DE replacement level for protocol 1 


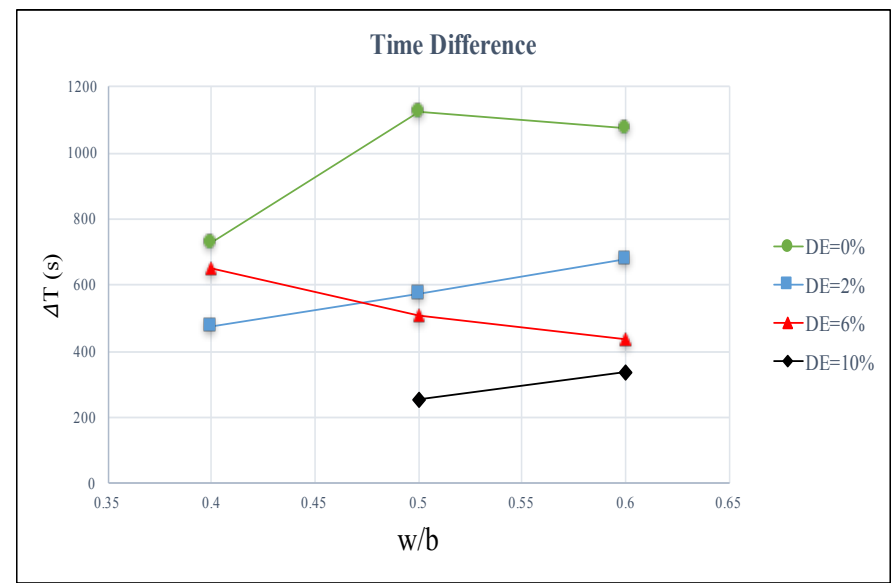

Figure 37: Time to reach to steady state against w/b ratio for protocol 1

To fully understand and explain the above results from protocol 1 , knowledge on the microscopic structure of cementitious pastes under shear rate is vital. At higher DE levels, solid volume fraction increases due to high water absorption of DE particles. This increase in solid volume fraction results in closer gaps between solid particles. These close gaps, especially between cement particles, leads to formation of a network of interconnected agglomerates at rest. When the high shear rate is applied, the resulted induced shear stress breaks this network and cause a quick and significant drop in shear stress. On the other hand, again due to high solid volume fraction, the inter-particle interaction between solid particles is high and thus, after a relatively short time, de-flocculation and flocculation rate reach to an equilibrium condition, i.e. cement paste reaches to its steady state. However, in lower DE levels, the gap between solid particles are larger and formation of such a fully interconnected network of agglomerates is more difficult. Therefore, de-flocculation happens in a lower rate and drop in shear stress is not as steep as pates with higher DE content. Also, due to lower inter-particle interaction between solid particles, it takes longer for these cementitious pastes to reach to their equilibrium condition. Therefore, overall, the 
breakdown energy required for cement pastes with higher DE content to reach to their steady state is lower than those with lower DE content.

Figure 38 and Figure 39, show static yield stress of all mix proportions against DE level and w/b ration from protocol 2 . For $0.6 \mathrm{w} / \mathrm{b}$ ratio, static yield stress has not changed significantly for different DE contents. For $0.5 \mathrm{w} / \mathrm{b}$ ratio, a slight increase for $6 \%$ and $10 \%$ replacement levels is observed, but it's not significant. For $0.4 \mathrm{w} / \mathrm{b}$ ratio, not much a difference is seen till $2 \%$ replacement, however, from 2 to $6 \%$ replacement level, static yield stress increases drastically. This is to some extent in agreement with breakdown energy from protocol 1 (Figure 32) which shows a slight increase from $2 \%$ to $6 \%$ at $0.4 \mathrm{w} / \mathrm{b}$ ratio. It can be due to very low water content of this cement paste caused by high DE level. From Figure 39, for all DE replacement levels, static yield stress has decreased with increase in $\mathrm{w} / \mathrm{b}$ ratio, as expected.

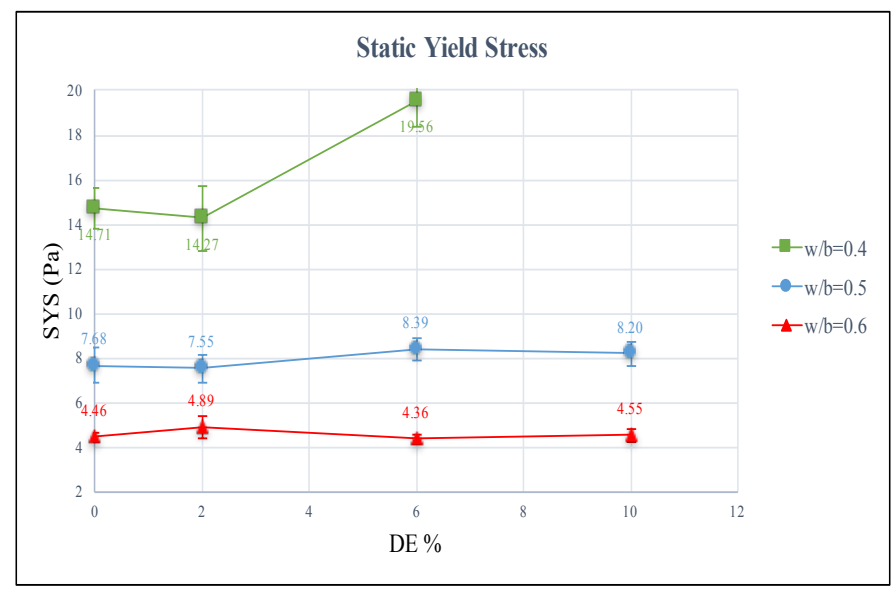

Figure 38: Static yield stress against DE content from protocol 2 


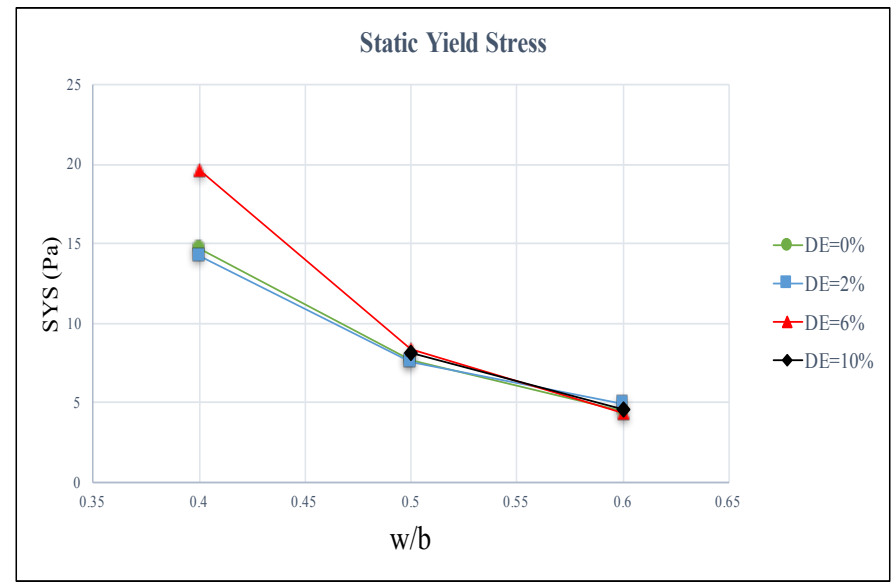

Figure 39: Static yield stress against $\mathrm{w} / \mathrm{b}$ ratio from protocol 2

It should also be mentioned that although the concept of protocol 1 and 2 are both related to breakdown aspect of thixotropy of cement pastes, they measure two different parameters. What measured in protocol 2 is the critical amount of shear that is needed to initiate the flow (i.e. the starting point of breakdown). While, protocol 1 measures the easiness to achieve the steady state (i.e. the required input energy for breakdown). By comparing protocol 1 and 2, it can be concluded that increase in DE content of cement pastes can decrease the amount of total energy required to breakdown cement pastes' structures to their most practically possible deflocculated state (steady state), however, the minimum shear stress needed to initiate the flow (structure breakdown) may not change significantly with variable DE replacement.

\subsubsection{Buildup}

Figure 40 shows build-up ratio (BR) against DE replacement level from protocol 3. Cementitious pastes with different $w / b$ ratio show different trends with increase in DE content. For 0.4 and $0.5 \mathrm{w} / \mathrm{b}$ ratios, an overall decrease in build-up ratio is observed when $\mathrm{DE}$ content is increased. While, for $0.6 \mathrm{w} / \mathrm{b}$ ratio, a significant increase in build-up ratio is observed from 0 to $6 \%$, then decreased when $10 \%$ of cement was replaced by DE. The 
observed phenomenon may be explained by solid volume fraction. When DE content is increased, it absorbs larger amount of free water, leaving less space in between particles that contributes to a higher flocculation and build-up ratio. When a threshold DE replacement level is surpassed (e.g. $6 \%$ for $w / b=0.4$ ), the repulsive forces from DE particles become dominant due to the shortened gap between solid particles, which reduce the buildup ratio. Figure 41 shows that the build-up ratio is the most sensitive to $6 \%$ of DE replacement, as the $B_{R}$ is kept as a constant when 0 or $2 \%$ of $D E$ is used.

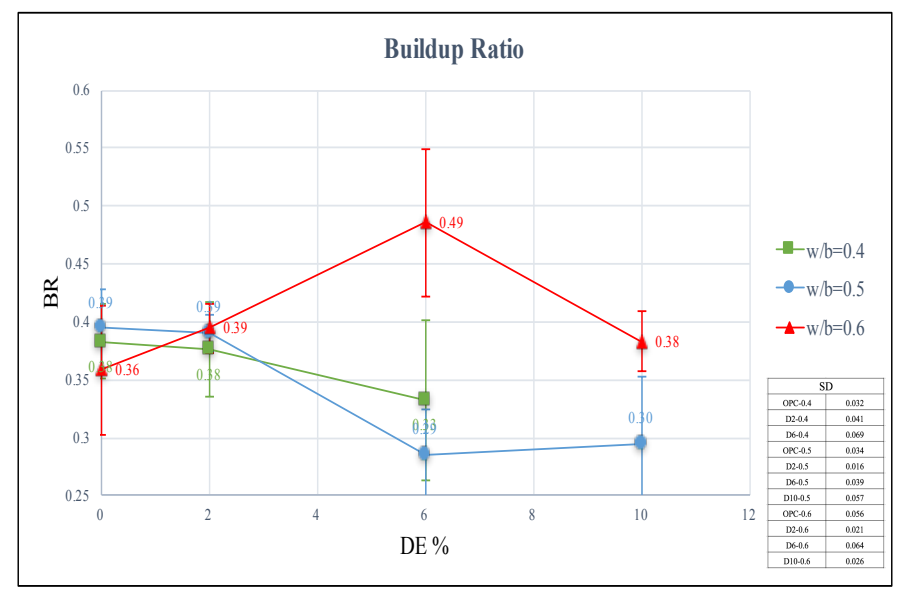

Figure 40: Build-up ratios from protocol 3 against DE replacement levels

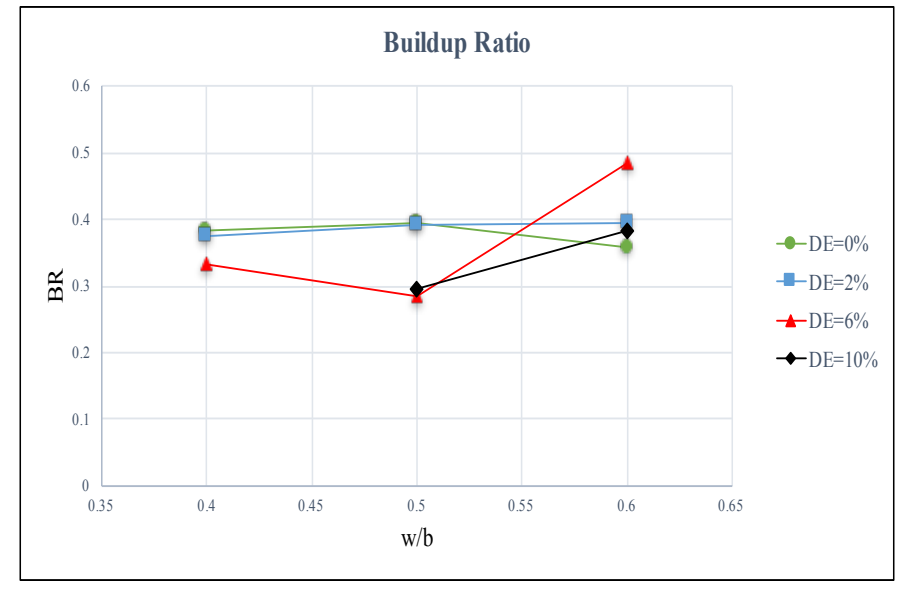

Figure 41: Build-up ratios from protocol 3 against different $w / b$ ratios

Figure 42 shows the build-up times of all mix proportions from protocol 3 against DE replacement levels. From the figure, for $w / b=0.5$ pastes, it takes longer to build up 
stresses when DE content is increased from 0 to $6 \%$, but once this threshold is passed, the buildup time decreases. Comparing the results from Figure 40 and Figure 42, a longer build-up time corresponds to a lower build-up ratio. This indicates roughly a linear trend between the build-up ratio and time.

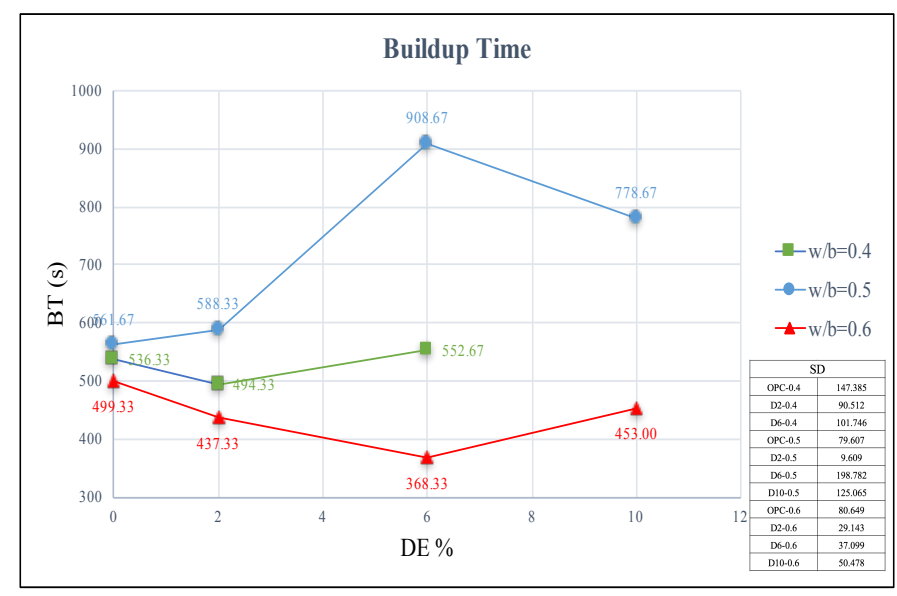

Figure 42: Build-up time from protocol 3 against DE replacement levels

Figure 43 and Figure 44 show build-up ratios from protocol 4 against DE replacement levels and w/b ratios, respectively. Again, for $0.6 \mathrm{w} / \mathrm{b}$ ratio, the build-up increases with DE content till $6 \%$ and then decreases. For 0.4 and $0.5 \mathrm{w} / \mathrm{b}$ ratios, an opposite trend is observed. Apart from the fact that OPC-0.5 and D2-0.5 show unexpectedly higher buildup ratios, the overall trends are in very good agreement with the results obtained from protocol 3. 


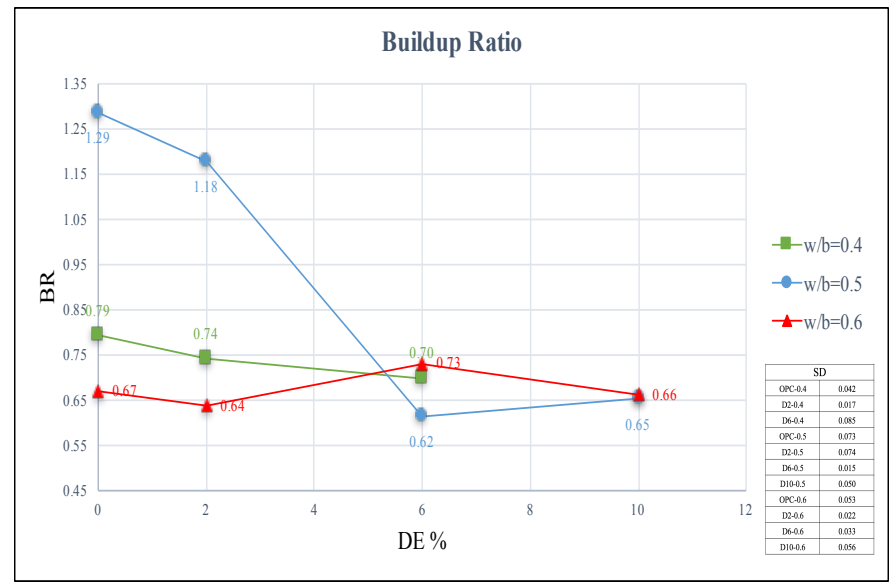

Figure 43: Build-up ratios from protocol 4 against DE replacement levels

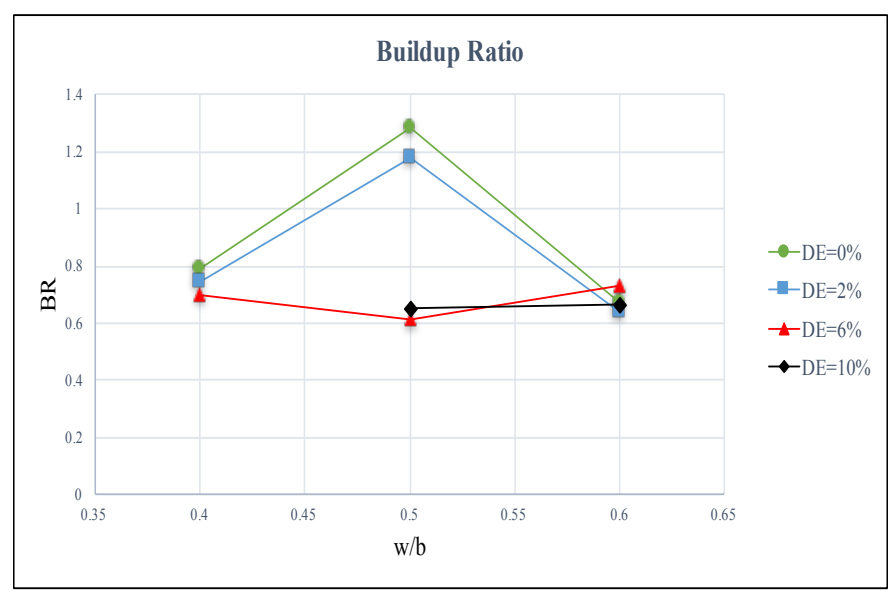

Figure 44: Build-up ratios from protocol 4 against different $w / b$ ratios

\subsubsection{Breakdown and build up correlation}

By comparing the results from protocol 1 and 2 (breakdown) with the results from protocol 3 and 4 (build-up), no clear correlation between breakdown and build-up aspects was observed. Both 0.5 and $0.6 \mathrm{w} / \mathrm{b}$ ratios show a drop in breakdown energy by increase in DE content from protocol 1 and no significant change in static yield stress from protocol 2. But when it comes to buildup ratios from protocol 3 and 4 , at least till $6 \%$ replacement level, $0.5 \mathrm{w} / \mathrm{b}$ ratio shows a continuous drop in build-up ratio, while $0.6 \mathrm{w} / \mathrm{b}$ ratio shows a 
continuous increase. Therefore, it can be concluded that to fully understand thixotropic behavior of cementitious pastes under different conditions, both breakdown and build-up aspects of their thixotropic behavior should be investigated. Any assumption based on only one of these two aspects can lead to misunderstanding of cementitious pastes characteristics.

\subsubsection{Temperature tests}

To investigate the temperature effect on thixotropy, protocol 2 and 4 were applied to pastes with $\mathrm{w} / \mathrm{b}$ ratios of 0.5 and 0.6 with curing temperature changing from $10^{\circ} \mathrm{C}$ to

$40^{\circ} \mathrm{C}$. Figure 45-Figure 48, show the obtained results from protocol 2. From Figure 46 and Figure 48, it is observed that for all cementitious pastes, independent of w/b ratio and/or DE replacement level, static yield stress increases with increase in temperature. In other words, increase in temperature makes it more difficult to initiate the flow of cementitious pastes. This can simply be due to increase in hydration rate caused by increase in temperature. Also, Figure 45 and Figure 47 show that trend of change in static yield stress by increase in DE content is not sensitive to temperature. For both $\mathrm{w} / \mathrm{b}$ ratios, 0.5 and 0.6 , the change in static yield stress shows almost similar trend for all three temperatures. By comparing Figure 45 and 46 (or 47 and 48), one can probably conclude that for a given w/b ratio, the thixotropic behavior of the paste is more sensitive to the temperature rather than the mix ingredient. 


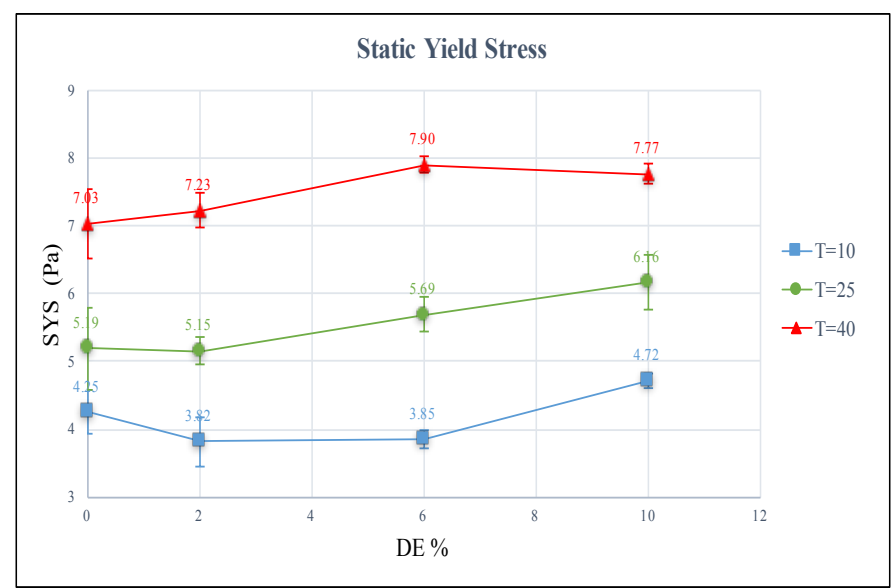

Figure 45: Static yield stress from protocol 2 against $D E$ replacement level $(w / b=0.5)$

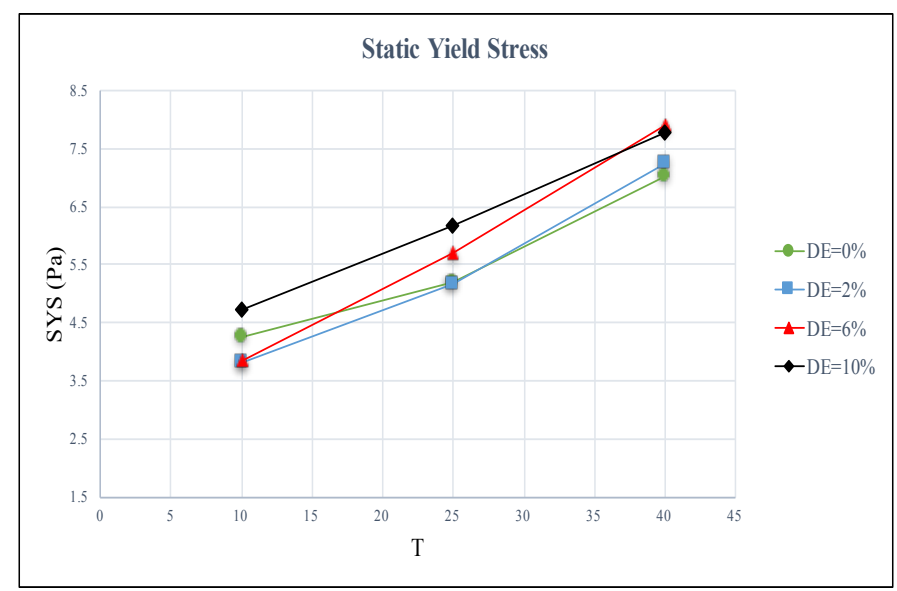

Figure 46: Static yield stress from protocol 2 against temperature $(w / b=0.5)$

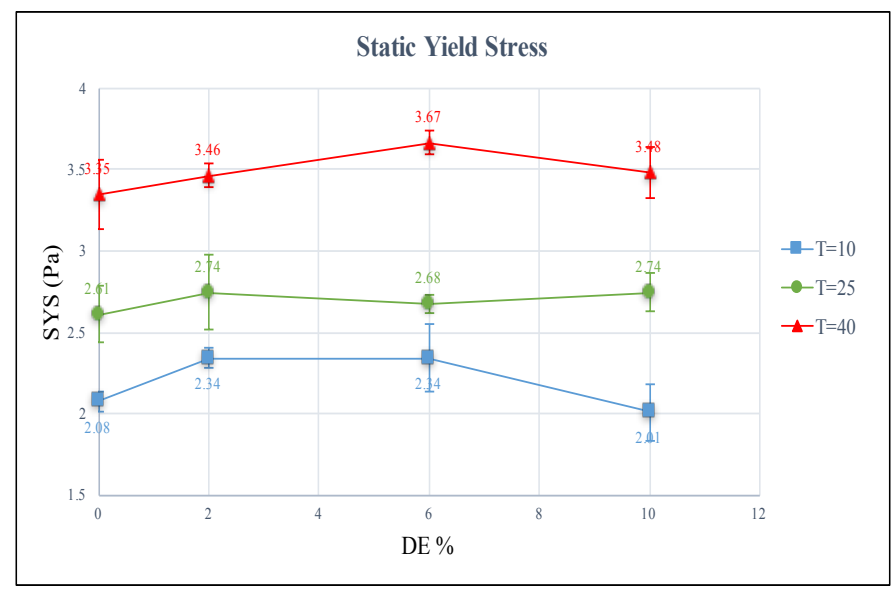

Figure 47: Static yield stress from protocol 2 against DE replacement level $(\mathrm{w} / \mathrm{b}=0.6)$ 


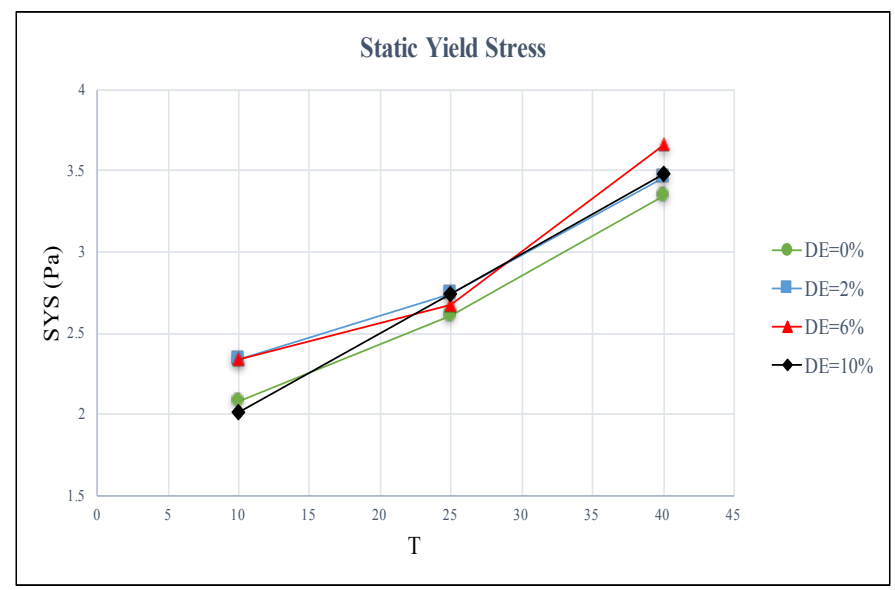

Figure 48: Static yield stress from protocol 2 against temperature $(\mathrm{w} / \mathrm{b}=0.6)$

The test results from protocol 4 are shown in Figure 50-Figure 55. From Figure 51 and Figure 53, for both 0.5 and $0.6 \mathrm{w} / \mathrm{b}$, buildup ratio is increased drastically for $40{ }^{\circ} \mathrm{C}$ compare to 10 and $25^{\circ} \mathrm{C}$. This can be explained by increase in hydration rate due to increase in temperature. For $0.5 \mathrm{w} / \mathrm{b}$ ratio, from 10 to $25^{\circ} \mathrm{C}$, buildup ratio shows no significant change; while for $0.6 \mathrm{w} / \mathrm{b}$ ratio, slight decrease in buildup ratio is observed. One possible explanation for this outcome may be the unique characteristics of water. At temperatures, close to $4{ }^{\circ} \mathrm{C}$, specific volume of water is at its minimum, see Figure 49 . Although at these temperatures hydration rate is expected to decrease but the closer gap between solid particles, due to lower specific volume of water, may compensate for that or even lead to higher buildup ratios. This impact can be seen clearer at $0.6 \mathrm{w} / \mathrm{b}$ ratio due to its higher water content. 


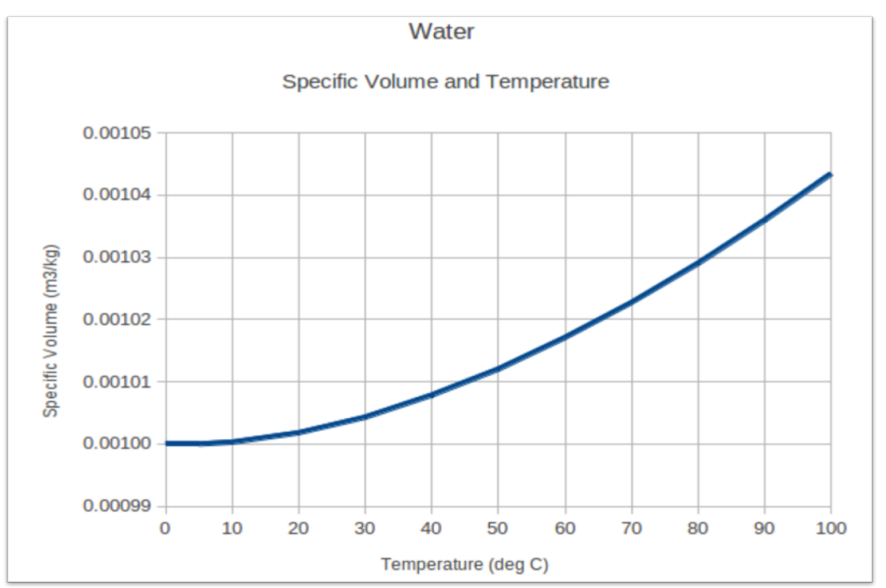

Figure 49: Specific volume of water against temperature

(figure from http://www.engineeringtoolbox.com/water-specific-volume-weight-d 661.html)

Figure 50 and Figure 52, show buildup ratio against DE content at different temperatures. For both w/b ratios, 0.5 and 0.6 , change in temperature shows minimum influence on overall trend of buildup ratio. For $0.5 \mathrm{w} / \mathrm{b}$, almost at all three temperatures, trends show an overall decrease in buildup ratio with increase in DE content; while for $0.6 \mathrm{w} / \mathrm{b}$, they show an increase.

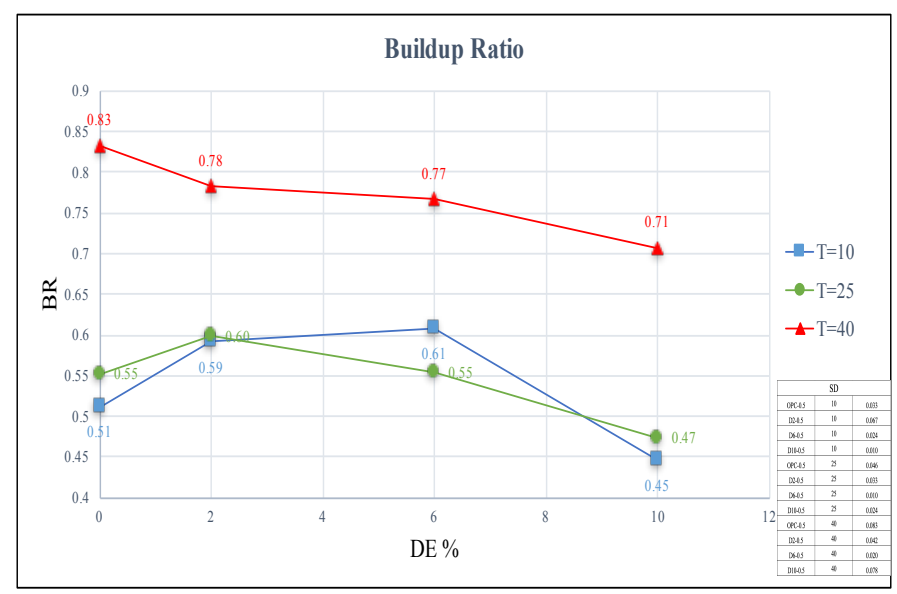

Figure 50: Build-up ration from protocol 4 against DE replacement level $(\mathrm{w} / \mathrm{b}=0.5)$ 


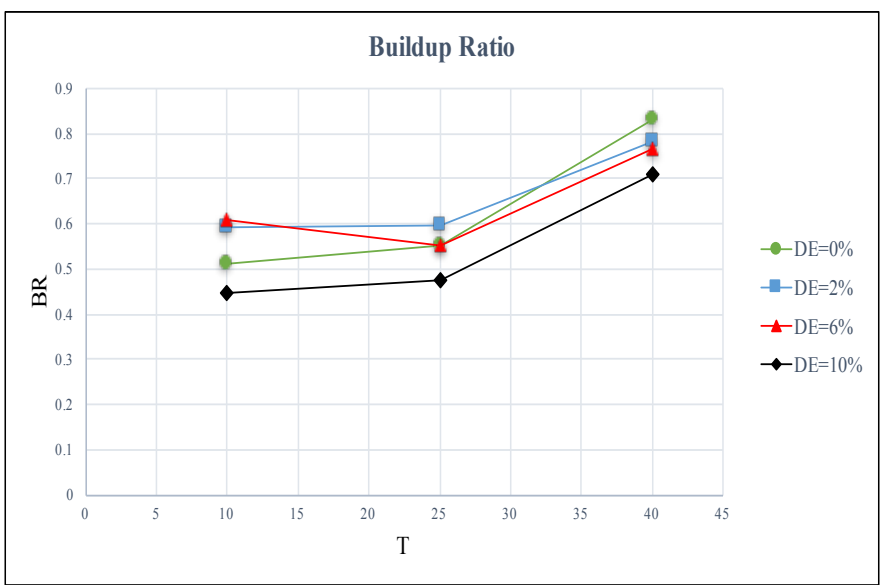

Figure 51: Build-up ratio from protocol 4 against temperature $(\mathrm{w} / \mathrm{b}=0.5)$

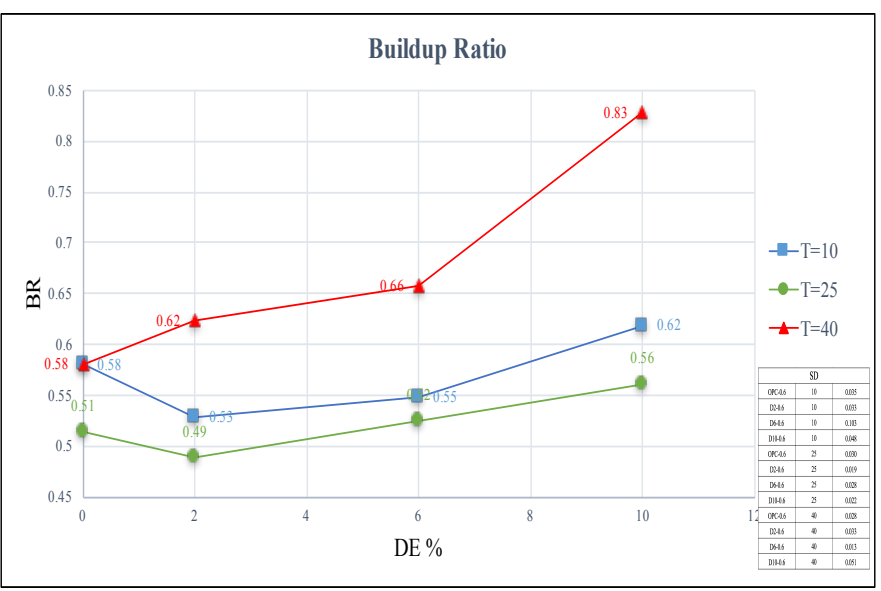

Figure 52: Build-up ratio from protocol 4 against $\mathrm{DE}$ replacement level $(\mathrm{w} / \mathrm{b}=0.6)$

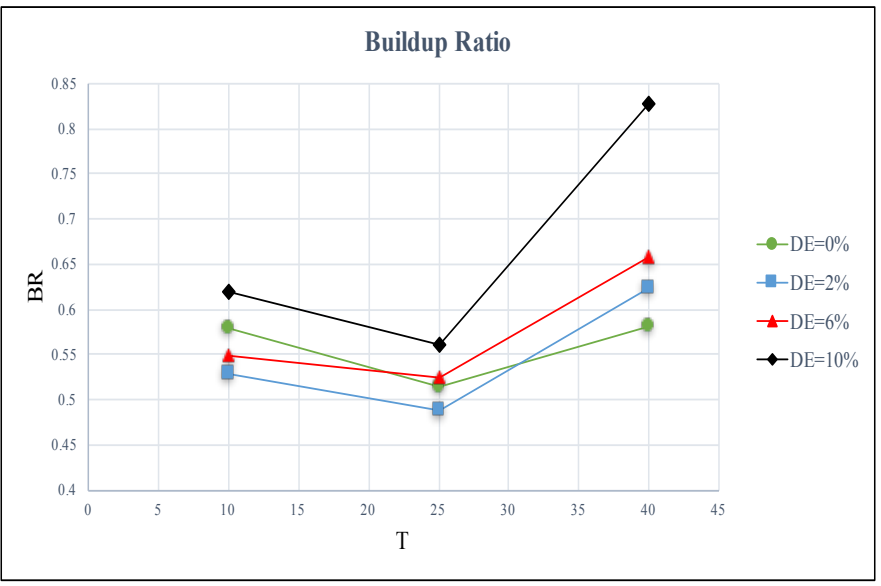

Figure 53: Build-up ratio from protocol 4 against temperature $(\mathrm{w} / \mathrm{b}=0.6)$ 


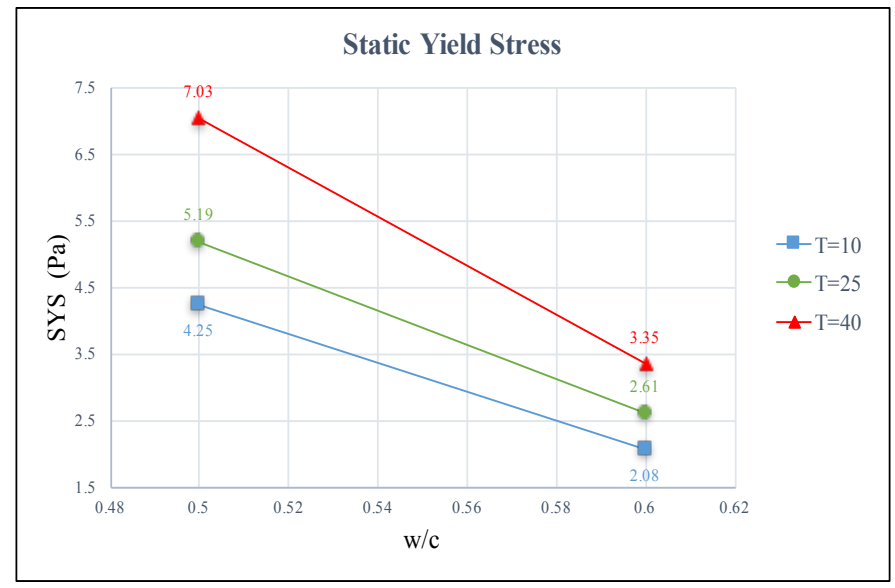

Figure 54: Static yield stress for OPC- 0.5 and OPC- 0.6 at different temperatures against water content

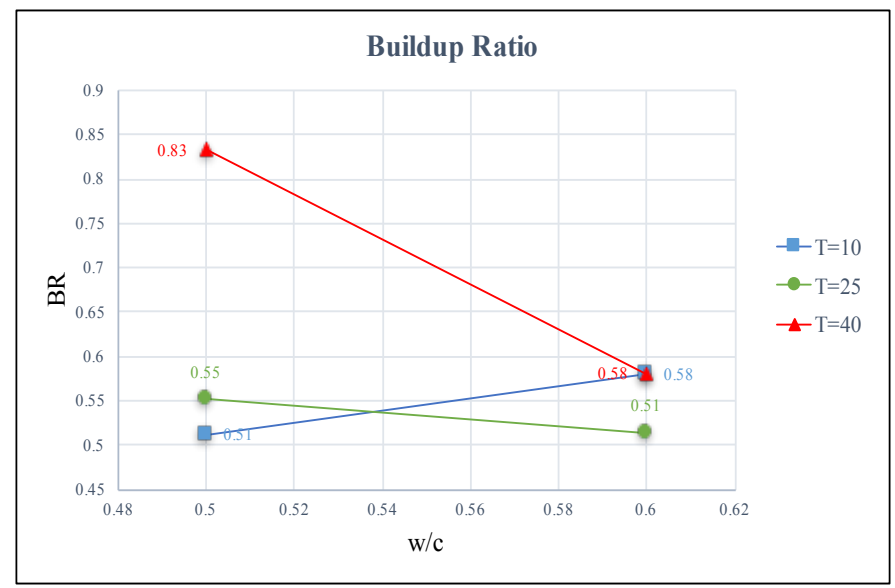

Figure 55: Build-up for OPC- 0.5 and OPC- 0.6 at different temperatures against water content

Figure 54 and Figure 55 show how static yield stress and buildup ratio change at different temperatures by change in water content (only for OPC-0.5 and OPC-0.6). For all three temperatures, increase in water content from 0.5 to $0.6 \mathrm{w} / \mathrm{c}$ ratio results in lower static yield stress, i.e. easier breakdown. Thus, it can be concluded that influence of water content over static yield stress is independent of temperature. Also, for 25 and $40{ }^{\circ} \mathrm{C}$, increase in water content leads to lower buildup ratio. However, for $10{ }^{\circ} \mathrm{C}$, it shows slightly higher buildup ratio. As explained earlier, this may be due to impact of water characteristics on cementitious pastes with higher water content at temperatures close to $4{ }^{\circ} \mathrm{C}$. It should be 
mentioned here, that higher "buildup ratio" does not necessarily indicate higher "absolute" buildup rate. Buildup ratio and buildup rate are two different concepts.

Overall, it can be concluded that increase in temperature results in acceleration of hydration and flocculation rate of cement pastes, which consequently leads to higher static yield stresses and build-up ratios.

\subsubsection{Pressure tests}

To mimic the influence of pumping speed or casting rate on the thixtropic behavior, protocol 2 and 4 were used to test the pastes of OPC-0.6 and D10-0.6. During pumping, concrete can be exposed to pressures as low as 60 bar to as high as 400 bar. Therefore, two pressure conditions of $20 \mathrm{MPa}(200 \mathrm{bar})$ and $10 \mathrm{MPa}(100 \mathrm{bar})$ were selected as reasonable pressures. Figure 56 and Error! Reference source not found. show static yield stress and buildup ratio of OPC-0.6 and D10-0.6 mix proportions under different pressures. It is observed that an increase in pressure from 0 to $20 \mathrm{MPa}$ has minor impact on yield stress or buildup ratio of these cement pastes. The minimal decrease in yield stress or build-up ratio can be due to slight de-flocculation caused by high pressure.

Overall, it can be concluded that the impact of high pressure up to $20 \mathrm{MPa}$ on thixotropic behavior of cement pastes with $0.6 \mathrm{w} / \mathrm{b}$ ratio is minimal and can be neglected. This conclusion is in good agreement with findings of Kim et al. (Kim, Kwon, Kawashima, \& Yim, 2017). 


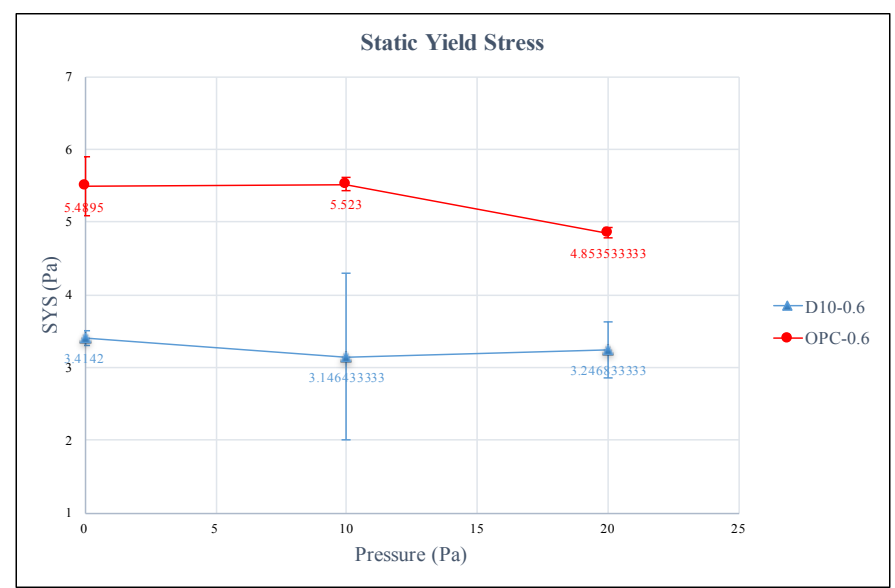

Figure 56: Static yield stress from protocol 2 at different pressures

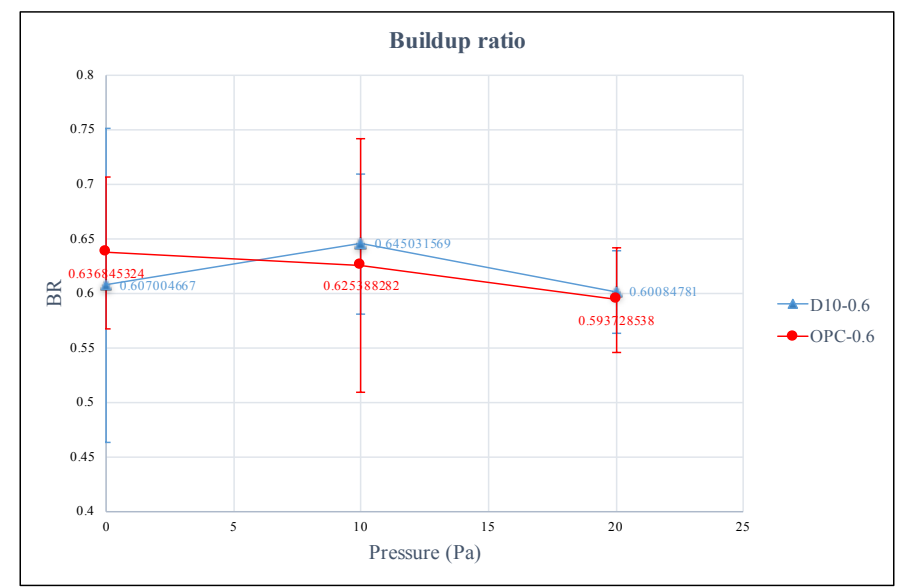

Figure 57: Build-up ratio from protocol 4 at different pressures

\subsection{Conclusions}

- Increase in DE content of cement pastes can decrease the amount of energy required to breakdown cement pastes structures to their most practically possible deflocculated state (steady state) under certain shear rate but does not significantly change minimum shear stress needed to initiate their flow (structure breakdown).

- No clear trend of build-up ratio of cement paste with different mix proportions was observed. 
- No clear correlation between breakdown and build-up aspects of different cement pastes was observed. Hence, for each cement paste with specific mix proportion both aspects should be investigated.

- Increase in temperature results in acceleration in hydration and flocculation rate of cement pastes, which consequently leads to higher static yield stress and build-up ratio.

- Impact of high pressure up to $20 \mathrm{MPa}$ on thixotropic behavior of cement pastes with $0.6 \mathrm{w} / \mathrm{b}$ ratio is minimal and can be neglected. 


\section{CHAPTER 5}

\section{THIXOTROPIC MODEL}

\subsection{Introduction}

As mentioned in previous chapters, thixotropic materials such as concrete show shear thinning behavior. Their internal structure breaks down under higher shear rates and builds up at lower shear rates or at rest. If a constant shear rate is applied long enough, material reaches to a so called "steady state" or "equilibrium condition". At equilibrium condition, rate of de-flocculation caused by applied shear and rate of flocculation due to inter-particle forces reach to an equilibrium. Due to this thixotropic behavior of concrete, there is a delay in concrete respond to any change in applied shear. The observed behavior caused by this delay is called transient behavior and exists between any two successive steady sates. Figure 58 shows the data obtained by a concrete rheometer compare to results expected from a simple yield stress fluid based on rheological models. Bold line shows what should be expected based on rheological models and thin line shows the real measurement obtained.

Rheological models such as Bingham are not suitable to describe the transient regime observed in thixotropic materials and may only be used to describe rheological behavior of concrete at steady state. Therefore, a thixotropic model capable of describing transient behavior of concrete can be very helpful to better understand and predict its behavior. 

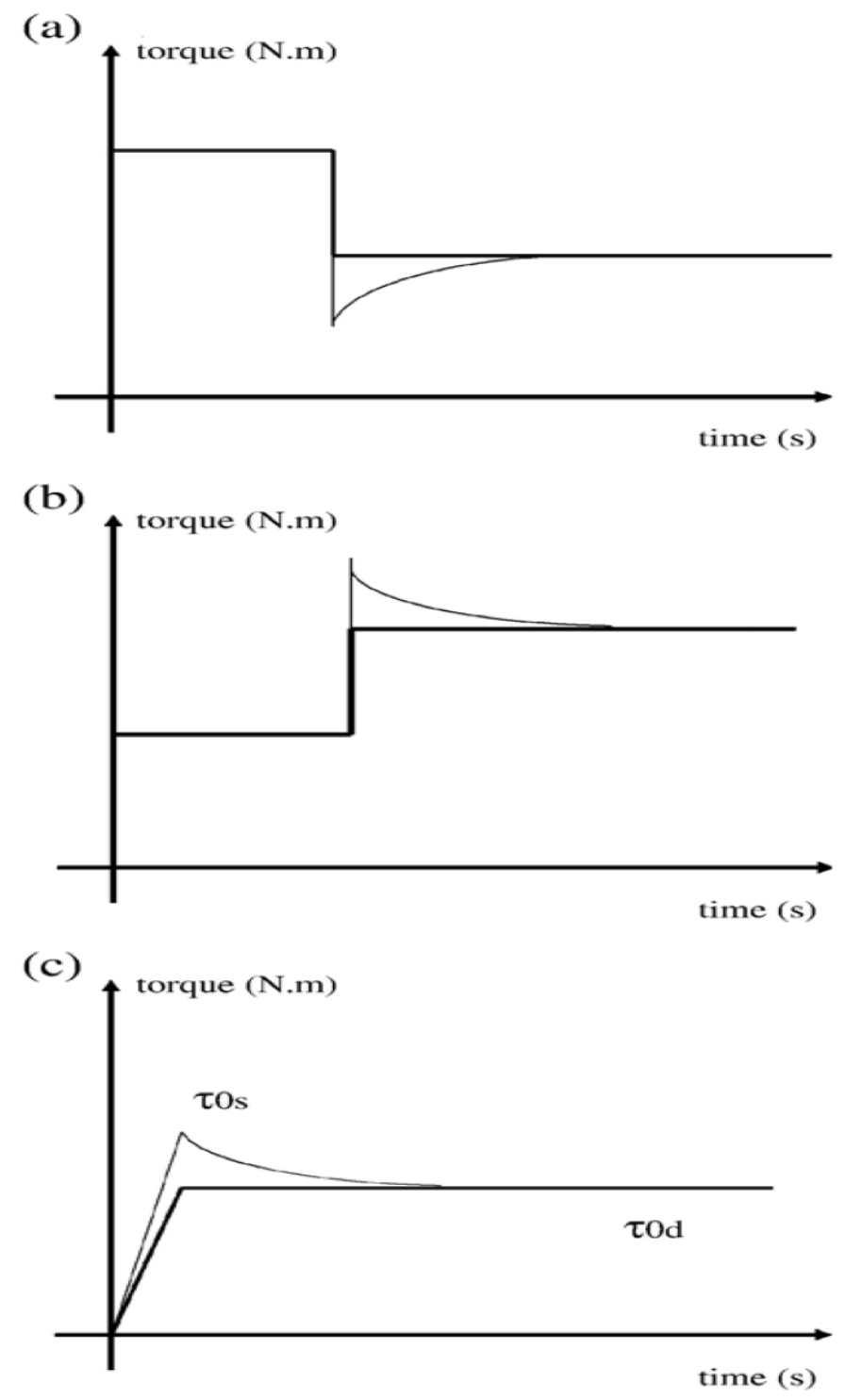

Figure 58: Examples of transient flow behaviors in practice against expected behavior from rheological models, figure from (Roussel, 2006)

However, proposing a thixotropic model for concrete can be challenging. Rheological behavior of concrete constantly alters due to hydration process which starts as soon as cement and water are mixed. Hydration is irreversible in concrete structure caused by chemical reaction between water and cement. While, thixotropy is reversible buildup and break down caused by flocculation and de-flocculation of cement particles. Separating the irreversible effect of hydration from reversible effect of thixotropy is complicated. Though, 
Jarny et al (Jarny et al., 2005) using MRI velocimetry have shown that over short periods of time, the irreversible effects of hydration processes is not significant and the reversible effects of thixotropy dominate. Therefore, it is reasonable to neglect irreversible evolution of concrete in intermediate periods up to $30 \mathrm{~min}$ (Roussel, 2006). This simplifies the problem and make it possible to model thixotropic behavior of concrete.

In this chapter, first the most common thixotropic model used for cementitious materials and a simplified version of that proposed by Roussel (Roussel, 2006) are described. Then, the accuracy of model is investigated by fitting it to the actual results obtained by a rheometer. A test procedure is determined and changes in thixotropic behavior of cement pastes are monitored at various resting times. Cement pastes with different mix proportion and at different temperatures are investigated. Finally, a comparison between cement pastes flocculation process at rest from obtained data and model's predictions are conducted and some modifications are proposed.

\subsection{Existing thixotropic model for cementitious materials}

The general form of existing models used for describing thixotropic behavior of cementitious materials, which is based on models suggested by (Cheng \& Evans, 1965) and (Coussot, Nguyen, Huynh, \& Bonn, 2002), is consisted of two parallel equations. It can be written as follows,

$$
\begin{gathered}
\tau=(1+\lambda) \tau_{0}+\mathrm{k} \dot{\gamma}^{n} \\
\frac{\mathbf{\partial} \lambda}{\mathbf{\partial} t}=\frac{1}{T \lambda^{m}}-\boldsymbol{\alpha} \lambda \dot{\gamma}
\end{gathered}
$$

Where $\lambda$ is current state of flocculation of material and $\mathrm{T}, \mathrm{m}$ and $\alpha$ are thixotropic parameters. 
The first equation shows the relationship between shear stress and applied shear rate depending on the current state of flocculation $(\lambda)$. The second equation, so called "kinetic equation", describes variation of $\lambda$ with time (t). Kinetic equation is based on the basic proposal that the rate of change of $\lambda$ is equal to difference between rate of natural flocculation of material and a rate of de-flocculation due to applied shear.

For a thixotropic model, suitable for describing flow behavior of concrete, scientific precision is not the only factor. The model should also be useful from a practical point of view. It should be simple; the number of parameters should be kept as low as possible and they should be easily measured. For this purpose, (Roussel, 2006) presented an alternative simplified version of the model. This simplified model has been used in new studies on thixotropic behavior of cementitious based materials (Ouyang, Tan, Corr, \& Shah, 2016; Qian \& Kawashima, 2016).

In his model, two assumptions were made. First, it was assumed that the Bingham model was sufficient to describe the steady state flow of fresh concrete ( $\mathrm{n}=1$ and $\mathrm{K}=$ plastic viscosity, $\left.\mu_{\mathrm{p}}\right)$. Second, it was assumed that the yield stress at rest increased linearly $(\mathrm{m}=0)$. Based on results of other studies, these assumptions seem to be reasonable for concretes and mortars (Assaad \& Khayat, 2004; Assaad, Khayat, \& Mesbah, 2003; Ovarlez \& Roussel, 2006).

Therefore, the simplified version of general model becomes,

$$
\begin{gathered}
\tau=(1+\lambda) \tau_{0}+\mu_{\mathrm{p}} \dot{\gamma} \\
\frac{\partial \lambda}{\partial \mathrm{t}}=\frac{1}{\mathrm{~T}}-\alpha \lambda \dot{\gamma}
\end{gathered}
$$

In this simplified model, $\mathrm{T}$ is characteristic time of flocculation and $1 / \boldsymbol{\alpha} \dot{\gamma}$ is considered as characteristic time of de-flocculation. At constant shear rate, rate of flocculation 
is insignificant compare to rate of de-flocculation; i.e. T is way longer than $1 / \boldsymbol{\alpha} \dot{\gamma}$ (Roussel, 2006). Therefore, it is reasonable to neglect the first part of kinetic equation. Thus, it becomes,

$$
\frac{\partial \lambda}{\partial t}=-\boldsymbol{\alpha} \lambda \dot{\gamma}
$$

After integration,

$$
\lambda=\lambda_{0} e^{-\alpha \dot{\gamma} \mathbf{t}}
$$

Where $\lambda_{0}$ is the state of flocculation at beginning of constant shear rate. By substituting $\lambda$ in simplified model, it is written,

$$
\tau=\left(1+\lambda_{0} e^{-\boldsymbol{\alpha} \dot{\gamma} \mathbf{t}}\right) \tau_{0}+\boldsymbol{\mu}_{p} \dot{\gamma}
$$

The model predicts an exponential decrease of the shear stress under constant shear rate with a de-flocculation characteristic time of $1 / \alpha \dot{\gamma}$.

At rest, the shear rate $(\dot{\gamma})$ is equal zero. Then, kinetic equation becomes,

$$
\frac{\partial \lambda}{\partial t}=\frac{1}{\mathrm{~T}}
$$

After integration,

$$
\lambda=\frac{t}{\mathrm{~T}}
$$

Therefore, by substituting $\lambda$ in simplified model, it is written,

$$
\tau_{0}(t)=(1+\lambda) \tau_{0}=\tau_{0}+\lambda \tau_{0}=\tau_{0}+\frac{\tau_{0}}{\mathrm{~T}} t
$$

Where $\mathrm{T}$ is characteristic time of flocculation and $\lambda \tau_{0}$ is thixotropic apparent yield stress. At rest, the model predicts that $\lambda$ and consequently $\tau_{0}$ increase linearly with time. 


\subsection{Experimental procedures}

In this study, Roussel's simplified model was used to monitor and analyze thixotropic behavior of cement pastes with DE. For this purpose, a protocol based on actual onsite condition has been developed. First, a reference point was determined as steady state, which is considered as the most de-flocculated state that can be achieved by concrete, i.e. $\lambda=0$. Studies show that the maximum shear rates in various steps of concert flow history are occurring during mixing and pumping. During these operations, concrete experiences shear rates of up to $60 \mathrm{~s}^{-1}$ (Roussel, 2006). However, it has been shown that during pumping, cement paste can be subjected to higher shear rates especially at lubricating layer, from $217 \mathrm{~s}^{-1}$ up to $924 \mathrm{~s}^{-1}$ (Kim et al., 2017). Since cement pastes are used in this study, $300 \mathrm{~s}^{-1}$ is considered as a reasonable maximum shear rate for steady state. Cement pastes with 0.5 $\mathrm{w} / \mathrm{b}$ and DE replacement levels of 0,2 and $6 \%$ were selected. Also, to better simulate the real on-site conditions, three temperatures of 10,25 and $40{ }^{\circ} \mathrm{C}$ were considered. Raw materials, mix proportions and mixing procedure are the same as those in Chapter 3 , see Table 3-Table 5.

\subsubsection{Test protocol}

Rheometer MCR 502 by Anton Parr and concentric geometry (cup and bob) was used. For details of rheometer and cup and bob geometry see chapter 2. To make sure sample is properly and uniformly placed in rheometer and there are no air voids, $2.5 \mathrm{~min}$ after end of mixing, a shear rate of $50 \mathrm{~s}^{-1}$ for $10 \mathrm{~s}$ was applied on sample. The low shear rate of $50 \mathrm{~s}^{-1}$ and short duration of $10 \mathrm{~s}$ were purposely chosen to assure applied shear would not disturb or break down cement pastes structure. Then, the tested cement pastes

were let to rest for different resting times of 5, 10, 20, 30 and $40 \mathrm{~min}$ (from the end of 
mixing). For each resting time a new sample is mixed and prepared to avoid the effects of shear history. After the assigned resting time, samples were subjected to a constant shear rate of $300 \mathrm{~s}^{-1}$ for $10 \mathrm{~min}$. Based on preliminary tests, $10 \mathrm{~min}$ was the average time needed for cements pastes to reach their equilibrium condition at $300 \mathrm{~s}^{-1}$ shear rate. Immediately after this step, a downward flow curve from $300 \mathrm{~s}^{-1}$ to $0 \mathrm{~s}^{-1}$ in $60 \mathrm{~s}$ was applied, Figure 59.

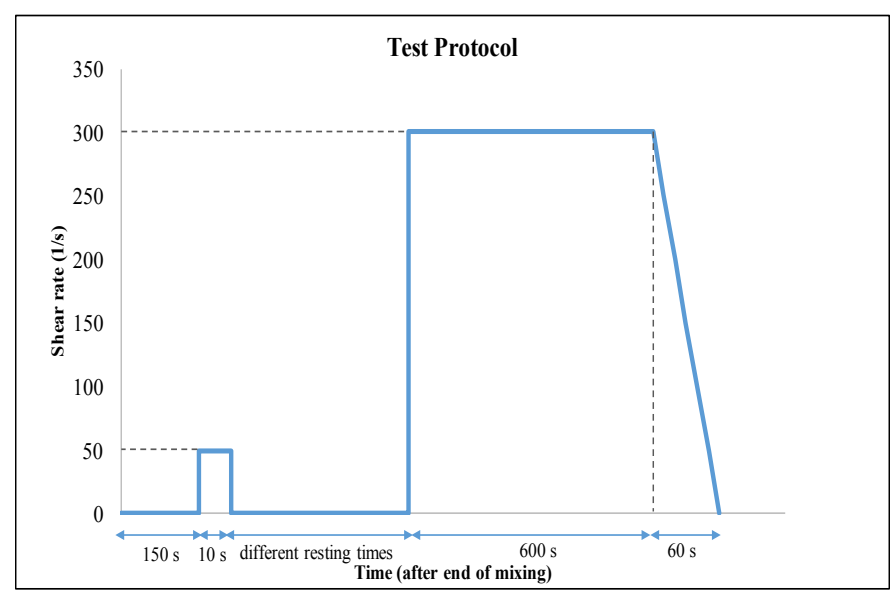

Figure 59: Test protocol procedures

\subsubsection{Steady state behavior}

At the end of $10 \mathrm{~min}$ constant shear rate of $300 \mathrm{~s}^{-1}$, cement pastes have reached to their steady state and it was assumed that current state of flocculation $(\lambda)$ equals zero. Therefore, Bingham model can be fitted to the flow curve to determine the rheological parameters of cement pastes at steady state, i.e. plastic viscosity $\left(\mu_{\mathrm{p}}\right)$ and yield stress $\left(\tau_{0}\right)$. These parameters were later used to fit the thixotropic model. 


\subsubsection{Flocculation process at rest}

Based on Roussel's proposed model, changes of "the state of flocculation at beginning of constant shear rate" $\left(\lambda_{0}\right)$ by resting time can be used to study flocculation process at rest. At the beginning of constant shear rate $t$ equals zero $(t=0)$. Thus, model can be written as:

$$
\tau(\mathrm{t}=0)=\left(1+\lambda_{0}\right) \tau_{0}+\boldsymbol{\mu}_{p} \dot{\gamma}
$$

Since the values of all other parameters are known, the value of $\lambda_{0}$ can be calculated. By calculating $\lambda_{0}$ values and plotting them against resting times, development of flocculation process at rest for different cement pastes can be evaluated.

\subsubsection{De-flocculation under constant shear rate}

Also, monitoring changes of $\alpha$ of de-flocculation characteristic time $(1 / \alpha \dot{\gamma})$ can be used to study de-flocculation process. Based on Roussel's simplified model changes of shear stress under constant shear rate can be shown as follows:

$$
\tau=\left(1+\lambda_{0} e^{-\boldsymbol{\alpha} \dot{\gamma} \mathbf{t}}\right) \tau_{0}+\boldsymbol{\mu}_{p} \dot{\gamma}
$$

Again, since values of all other parameters are known, thixotropic model can be fitted to actual data obtained from rheometer and best value of $\alpha$ is determined. An example of fitted model to experimental data obtained by rheometer is shown in Figure 60. Then, values of $\alpha$ plotted against resting time and changes in de-flocculation process of different cement pastes by resting time was investigated. 


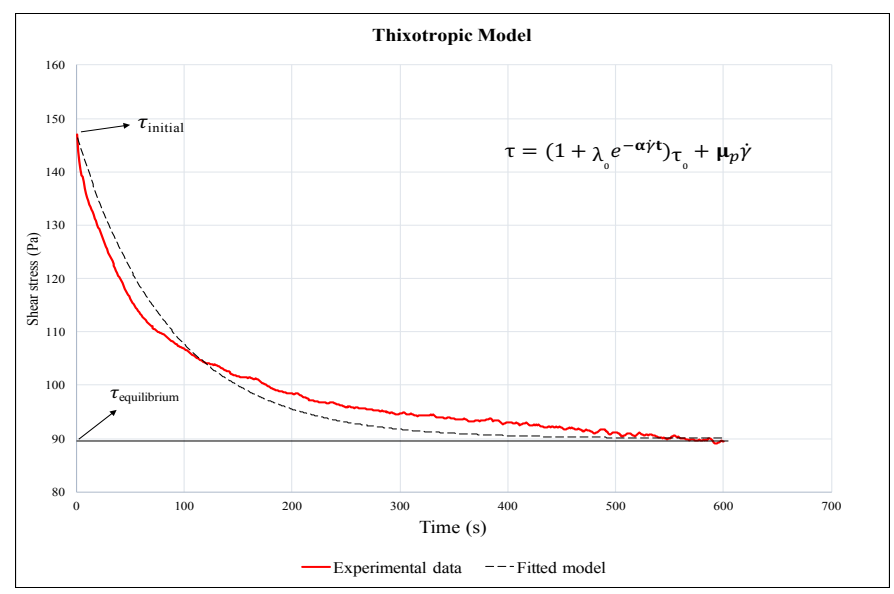

Figure 60: Example of fitted model to experimental data

1.22. Experimental results and discussion

\subsubsection{Flocculation process of cement pastes with different mix proportions}

Evolution of "the state of flocculation at beginning of constant shear rate" $\left(\lambda_{0}\right)$ by resting time for cement pastes with $0.5 \mathrm{w} / \mathrm{b}$ ratio and DE replacement levels of 0,2 and $6 \%$ are investigated. Figure 61 and Figure 62 show the evolution of $\lambda_{0}$ by resting time for different mix proportions. For all cement pastes, state of flocculation increases by resting time. Also, at all different resting times pastes with higher DE contents show higher state of flocculation. The Roussel's model predicts a linear increase in state of flocculation by resting time. This seems to be a fair assumption for concrete and mortar (Assaad \& Khayat, 2004; Assaad et al., 2003; Ovarlez \& Roussel, 2006), however, in case of cement pastes it is not very accurate (Ouyang et al., 2016). This can be due to the absence of aggregates and sands in cement pastes.

It is observed here that the evolution of $\lambda_{0}$ by resting time is not linear, especially at first 10 to $20 \mathrm{~min}$. It can be concluded that the formation of flocculation network occurs quicker in first few minutes and then gradually decreased by time. The evolution of $\lambda_{0}$ by resting time shows a convergence trend and can be well fitted to the following equation: 


$$
\lambda(\mathrm{t})=\lambda_{00}+\lambda_{u} \frac{K t}{K t+1}
$$

Where $\lambda_{00}$ is the initial state of flocculation (right after mixing); $\mathrm{K}$ is rate of flocculation and $\lambda_{u}$ is the ultimate state of flocculation. An example of fitted equation to experimental data is shown in Figure 63. Ultimate state of flocculation is a parameter used to fit the equation to experimental data and due to influence of hydration in longer periods of time such an ultimate flocculation state does not exist in practice. However, these parameters $\left(\lambda_{00}, \mathrm{~K}\right.$ and $\left.\lambda_{u}\right)$ can be used to quantitatively characterize flocculation process of cement pastes at short periods of time. Changes in $\lambda_{00}, \lambda_{u}$ and $\mathrm{K}$ against $\mathrm{DE}$ content is shown in Figure 64, Figure 65 and Figure 66, respectively.

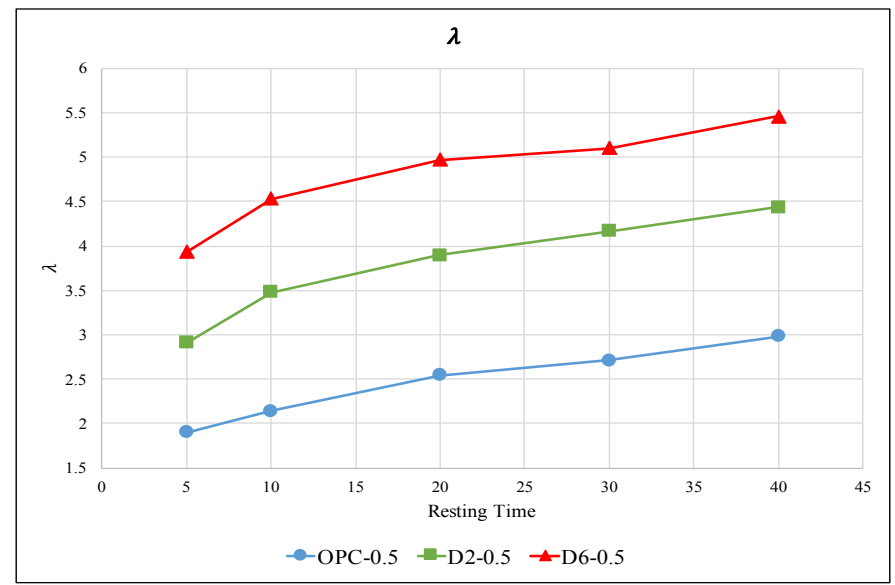

Figure 61: Evolution of $\lambda_{0}$ by resting time for different mix proportions 


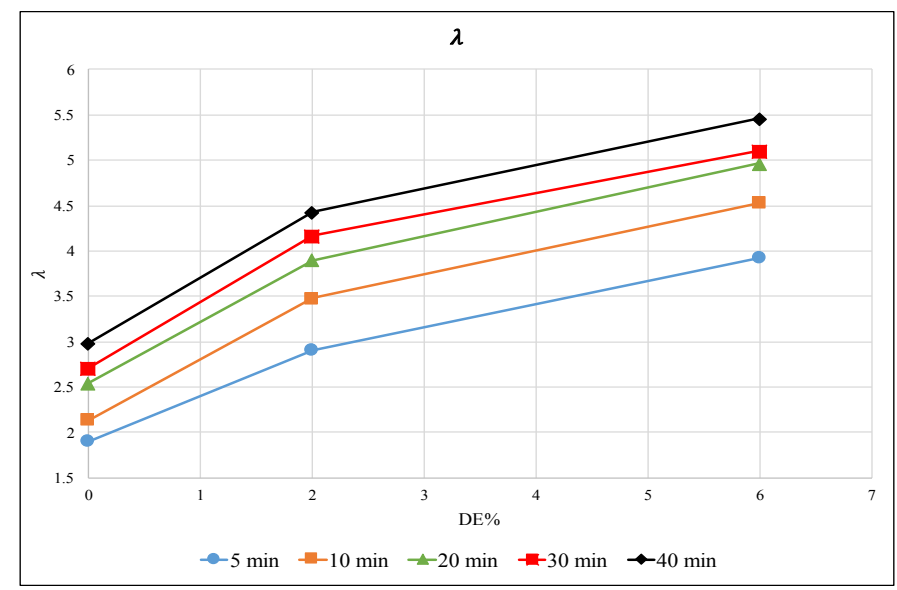

Figure 62: Evolution of $\lambda_{0}$ against DE replacement level at different resting times

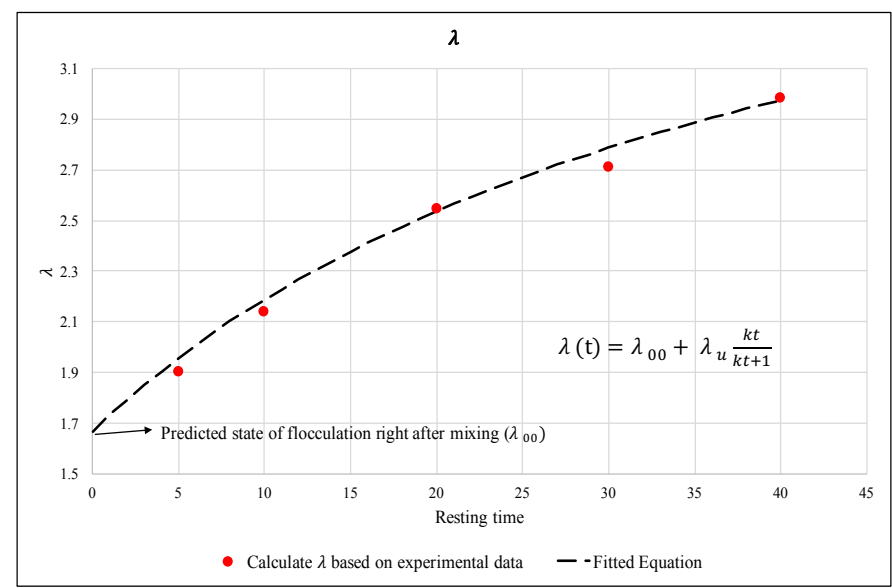

Figure 63: Fitted equation to experimental data for $\mathrm{OPC}-0.5$ at $25^{\circ} \mathrm{C}$

The values of $\lambda_{00}$ is the value extrapolated by equation for state of flocculation right after mixing $(t=0)$. The maximum de-flocculated status $(\lambda=0)$ is assumed to be at the end of constant shear rate of $300 \mathrm{~s}^{-1}$, i.e. steady state. Therefore, state of flocculation at any other point is higher than zero. Thixotropy of cement paste is consisted of a short-term phase due to colloidal flocculation and a long-term phase due to ongoing hydration nucleation. The time needed to form the network of colloidal interactions between cement particles is of the order of couples of seconds (Roussel, Ovarlez, Garrault, \& Brumaud, 2012). 
Therefore, the value of $\lambda_{00}$ can be associated with the colloidal flocculation of cement pastes.

It is also observed that the value of $\lambda_{00}$ increases by increase in DE content. It can be explained by the high Blaine surface and porous structure of DE particles which tend to absorb free water available in the paste. Although, this water will be released back to cement matrix in later ages, in early age, it causes reduction in water content. It should also be noticed that the specific gravity of DE is lighter than cement. When cement is replaced by $\mathrm{DE}$ with the same mass, the solid volume fraction increases. The increased solid volume fraction together with the porous texture of DE result in higher inter-particles interactions and consequently higher $\lambda_{00}$ value.

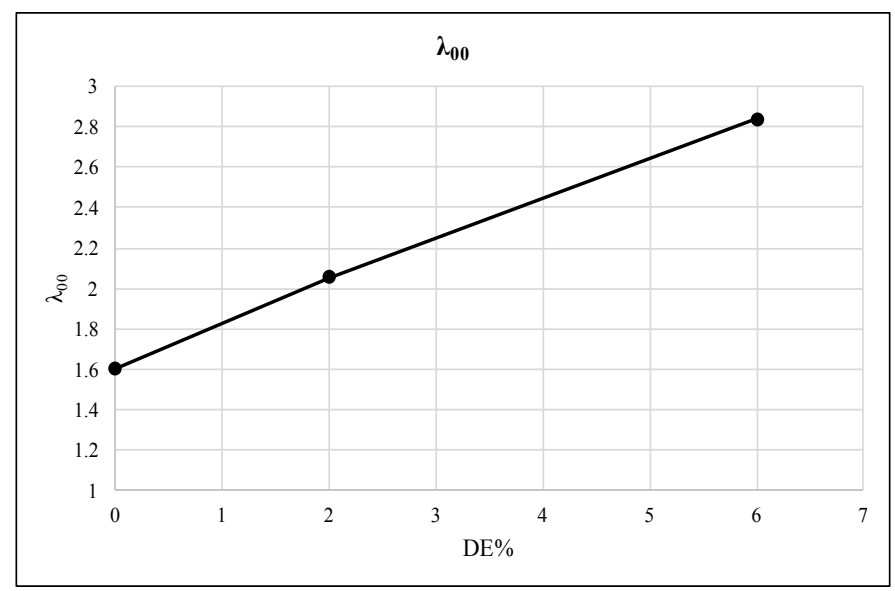

Figure 64: Initial state of flocculation $\left(\lambda_{00}\right)$ of cement pastes

The values of both $\lambda_{u}$ and $\mathrm{K}$ also increase with the increase in DE content of cement pastes. However, the rate of increase in these parameters drops significantly from 2 to $6 \%$ DE replacement level. Ultimate state of flocculation $\left(\lambda_{u}\right)$ is the maximum value that state of flocculation can develop over initial state of flocculation $\left(\lambda_{00}\right)$. As stated earlier, such a limit does not exist in practice and it is only used to characterize flocculation trend at short 
periods of time when irreversible influence of hydration can be neglected. And, $\mathrm{K}$ is the rate of flocculation which indicates how quickly ultimate state of flocculation can be reached. These values can be related to both short-term phase of thixotropy due to colloidal flocculation and long-term phase due to ongoing hydration nucleation. Thus, it can be concluded that increase in DE replacement level to some extent increases solid volume fraction and consequently increases colloidal interaction within cement particles and hydration nucleation. This results in higher initial state of flocculation and higher rate of flocculation. However, rate of these increase in flocculation evolution decreases by increase in level of $\mathrm{DE}$ replacement level. If the level of DE replacement level exceeds an optimum level it will decrease thixotropic properties of cement pastes due to low level of cement particles in cement pastes and negatively affects flocculation process.

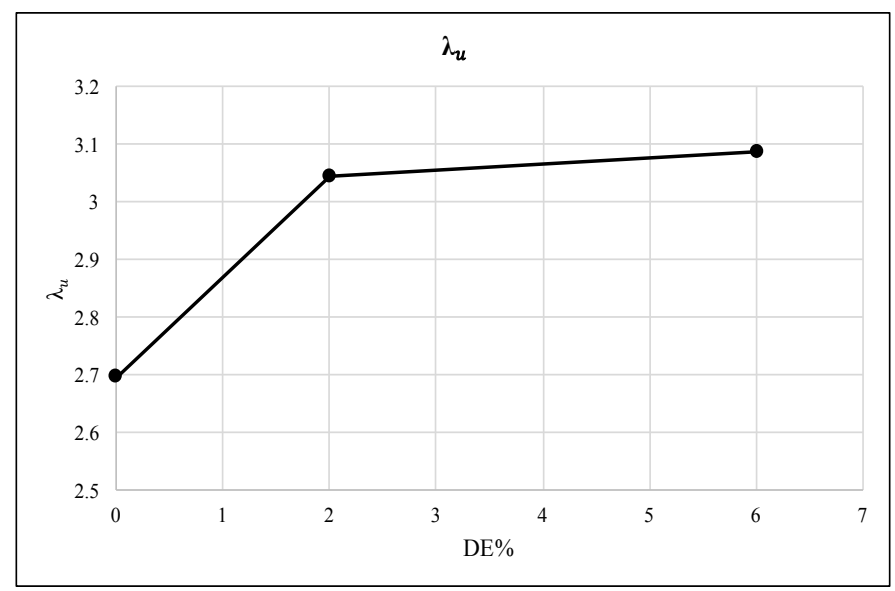

Figure 65: Ultimate state of flocculation $\left(\lambda_{\mathrm{u}}\right)$ of cement pastes 


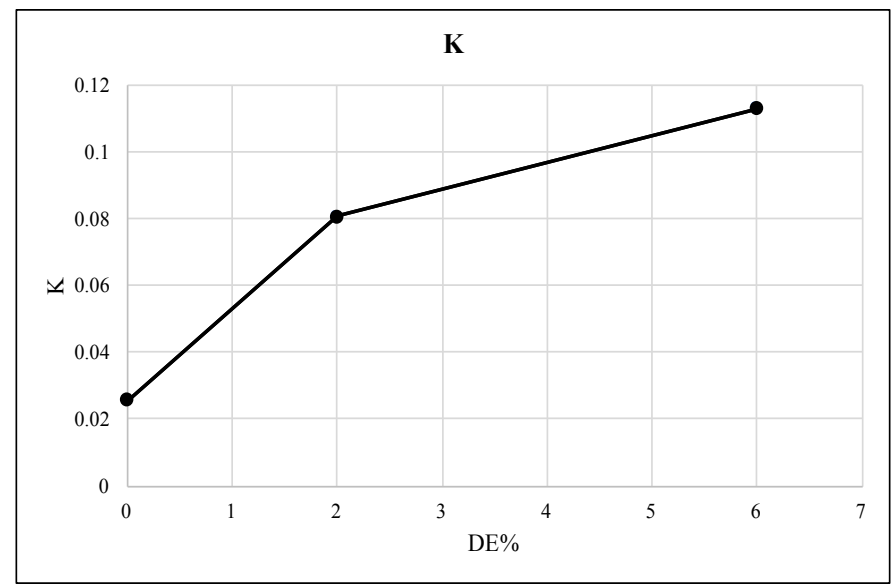

Figure 66: rate of flocculation $(\mathrm{K})$ of cement pastes

\subsubsection{De-flocculation process of cement pastes with different mix proportions}

Evolution of $\alpha$ of "de-flocculation characteristic time" (1/ $\alpha \dot{\gamma})$ by resting time for cement pastes with $0.5 \mathrm{w} / \mathrm{b}$ ratio and DE replacement levels of 0,2 and $6 \%$ were also investigated. Figure 67 and Figure 68 show the evolution of $\alpha$ by resting time for different mix proportions. The higher the value of $\alpha$ the shorter is the de-flocculation characteristic time which indicates quicker and easier de-flocculation to steady state. In general, for all cement pastes, $\alpha$ increases by resting time. At 5 min resting time, cement pates with higher DE content show higher values for $\alpha$, i.e. higher de-flocculation rate. However, by increase in resting time this pattern changes.

To explain the observed results once again two phases of thixotropy of cement pastes should be considered. At short resting times, thixotropy is mainly dominated by colloidal interactions such as van der Waals forces within cement particles and the flocculation network formed by these forces are easy to break. However, as resting time increases thixotropy of cement pastes is mostly governed by hydration nucleation which originates from the colloidal forces. Colloidal forces ease formation of a network of short CSH 
bridges between cement particles. Although the degree of hydration at this time is close to zero, the consequences of the CSH nucleation are strong at a macroscopic level as they nucleate specifically in very crucial zones in the interacting particles network (Roussel et al., 2012)

It has however to be kept in mind that thixotropy is defined at a purely macroscopic level. As long as shear is sufficient to bring the material back to a reference state, i.e. it is reversible in macroscopic scale, one does not have to care whether the evolution of the material behavior is due to colloidal flocculation or to CSH bridges between particles (Roussel et al., 2012).

Therefore, it can be concluded that for short resting times cement pastes with higher DE content show higher de-flocculation rate because of their higher state of flocculation caused by colloidal forces. However, at longer resting times, this high colloidal forces and high solid volume fraction compare to cement pastes with less DE content results in stronger hydration nucleation bonds and consequently lower de-flocculation rate.

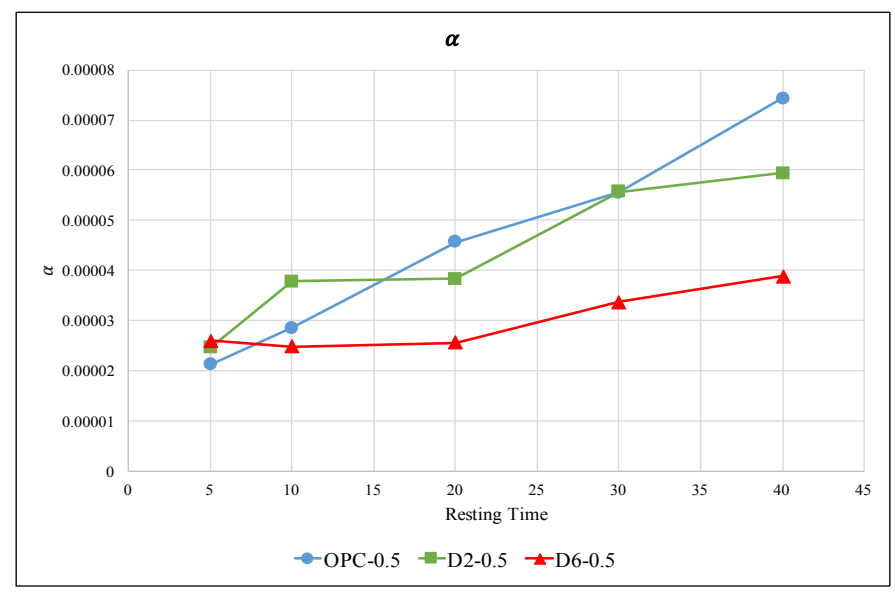

Figure 67: Evolution of $\alpha$ by resting time for different mix proportions 


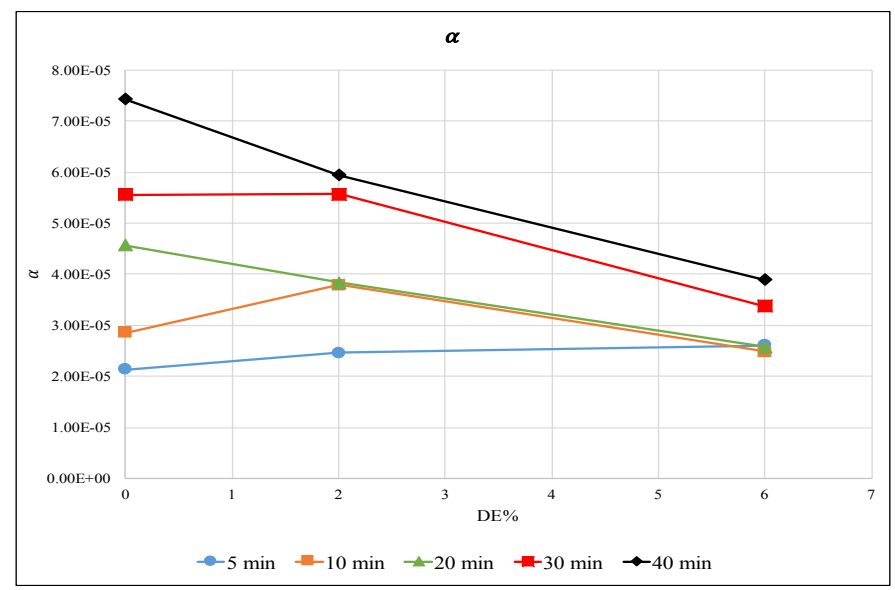

Figure 68: Evolution of $\alpha$ against DE replacement level at different resting times

\subsubsection{Influence of temperature on flocculation and de-flocculation process of cement} paste

Evolution of $\lambda_{0}$ and $\alpha$ by resting time for cement paste with $0.5 \mathrm{w} / \mathrm{c}$ ratio (OPC- 0.5$)$ at 10 , 25 and $40^{\circ} \mathrm{C}$ are investigated. Figure 69 and Figure 70 show the evolution of $\lambda_{0}$ by resting time at different temperatures. Up to 20 min resting time, no significant change in state of flocculation is observed. However, after 20 minutes $\lambda_{0}$ increases significantly by increase in temperature.

In addition, it was observed that evolution of state of flocculation $\left(\lambda_{0}\right)$ by time at $10{ }^{\circ} \mathrm{C}$, like $25{ }^{\circ} \mathrm{C}$, could be well fitted to equation $5-13$. However, at $40{ }^{\circ} \mathrm{C}$ evolution of $\lambda_{0}$ by time shows a concave trend with increasing slopes which can be better fitted to an equation as follow,

$$
\lambda(\mathrm{t})=\lambda_{00}+K t^{n}
$$

Where again, $\lambda_{00}$ is the initial state of flocculation (right after mixing); $\mathrm{K}$ indicates rate of flocculation and $\mathrm{n}$ is a number larger than 1.

Therefore, it can be concluded that increase in temperature of cement paste gradually changes its structural buildup pattern at rest, from a convergence trend to a concave 
trend with increasing slopes. This change is especially more obvious at temperatures higher than $25^{\circ} \mathrm{C}$ and resting times higher than $20 \mathrm{~min}$. As mentioned earlier, at short resting times colloidal flocculation dominates thixotropy of cement pastes. It seems that colloidal flocculation is less sensitive to change in temperature. However, at longer resting times hydration nucleation governs thixotropy of cement pastes which is more sensitive to temperature change. Therefore, the observed result can be explained by increase in rate of hydration nucleation due to increase in temperature.

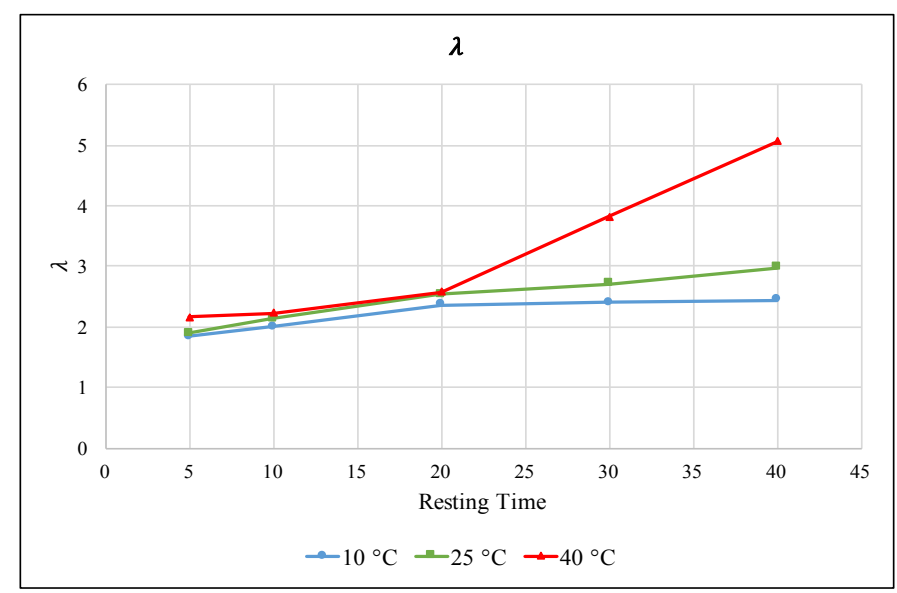

Figure 69: Evolution of $\lambda_{0}$ by resting times at different temperatures

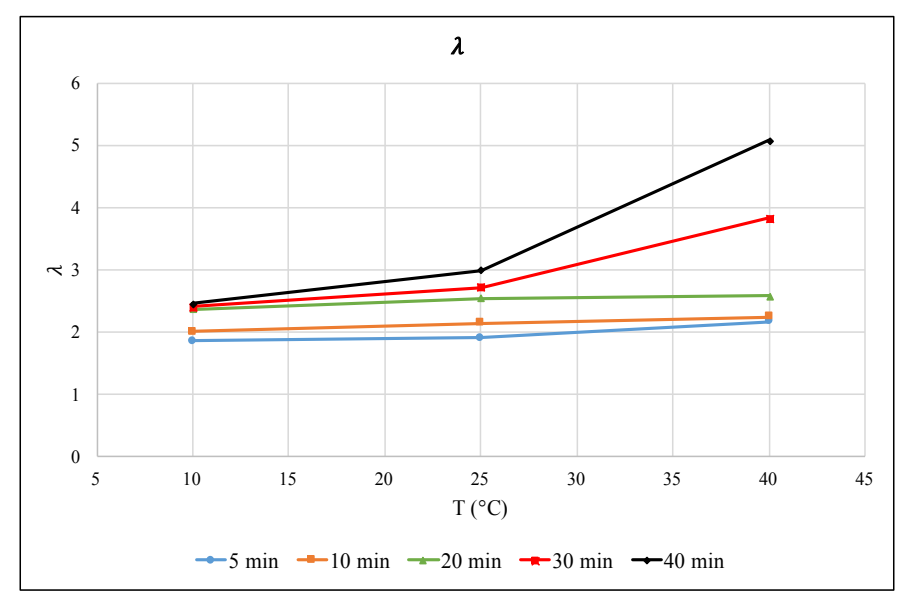

Figure 70: Evolution of $\lambda_{0}$ against temperature at different resting times 
Figure 71 and Figure 72 show the evolution of $\alpha$ by resting time at different temperatures. Again, it is observed that up to 20 min resting time, changes in de-flocculation process is less sensitive to changes in temperatures. But for longer resting times, $\alpha$ increases drastically. Two reasons can be mentioned for the obtained results. Firstly, due to increase in temperature and consequently rate of hydration nucleation, the state of flocculation at longer resting times (above $20 \mathrm{~min}$ ) is significantly higher than shorter resting time. This results in a high rate of de-flocculation at very beginning of shear decay under constant shear rate. Secondly, due to higher rate of hydration at higher temperatures, after $20 \mathrm{~min}$ the irreversible influence of hydration elevates the level of steady state. This accelerates reaching to equilibrium condition and increases the value of $\alpha$.

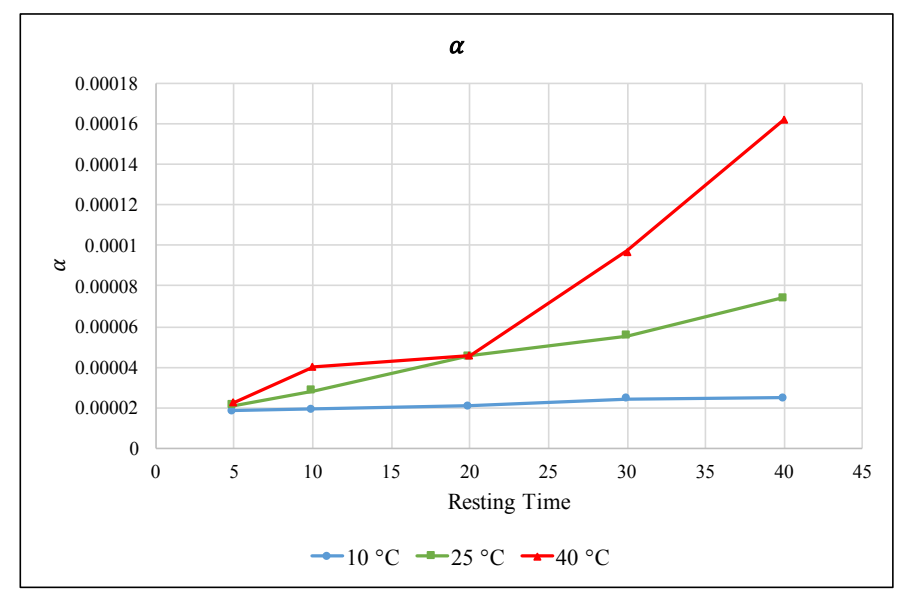

Figure 71: Evolution of $\alpha$ by resting times at different temperatures 


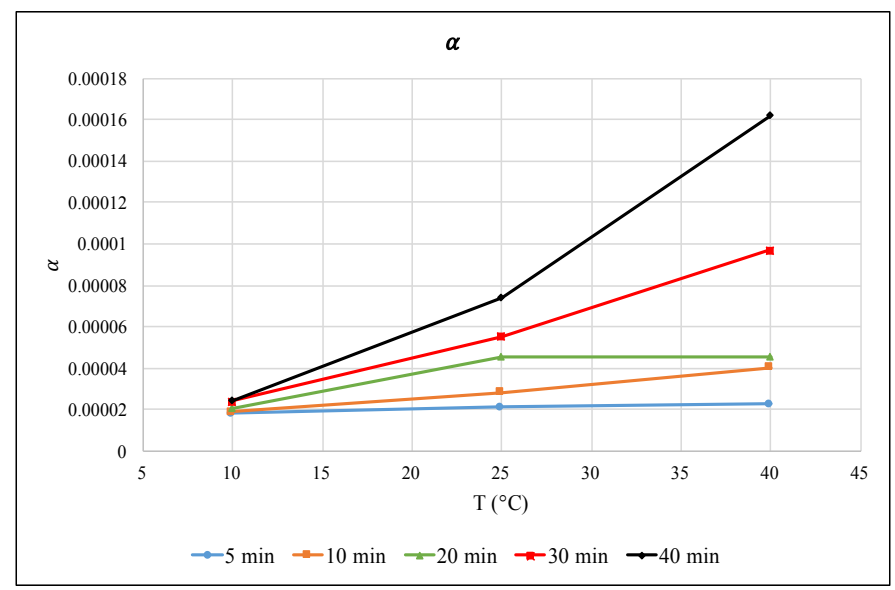

Figure 72: Evolution of $\alpha$ against temperature at different resting times

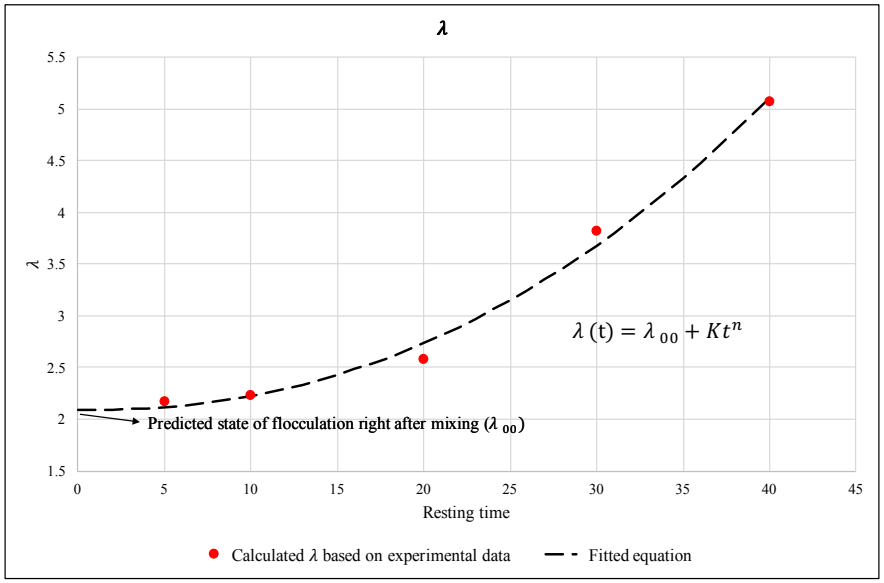

Figure 73: Fitted equation to experimental data for OPC- 0.5 at $40{ }^{\circ} \mathrm{C}$

\subsection{Conclusions}

- In general, the simplified thixotropic model proposed by Roussel is a reliable and practical platform to predict and monitor thixotropic behavior of cementitious materials.

- The simplified model seems to be more accurate for concrete and mortar but in case of cement paste some modifications based on mix proportion may be needed.

- The flocculation process of cement paste is not linear, especially at short resting times. 
- Increase in DE replacement level to some extent, increases solid volume fraction and consequently increases initial state of flocculation and rate of flocculation. However, rate of increase in flocculation evolution decreases by increase in level of DE replacement level.

- If the level of DE replacement level exceeds an optimum level it will decrease thixotropic properties of cement pastes due to low concentration of cement particles in cement pastes and negatively affects flocculation process.

- For short resting times cement pastes with higher DE content show higher deflocculation rate because of their higher state of flocculation caused by colloidal forces. However, at longer resting times, higher colloidal forces and higher solid volume fraction compare to cement pastes with less DE content results in stronger hydration nucleation bonds and consequently lower de-flocculation rate.

- Up to 20 min resting time, slight increase in state of flocculation is observed by increase in temperature. After 20 min, state of flocculation increases significantly with increase in temperature.

- increase in temperature of cement paste gradually changes its structural buildup pattern at rest, from a convergence trend to a concave trend with increasing slopes. This change is especially more obvious at temperatures higher than $25^{\circ} \mathrm{C}$ and resting times higher than $20 \mathrm{~min}$.

- Up to 20 min resting time, changes in de-flocculation process is less sensitive to changes in temperatures. But for longer resting times, $\alpha$ increases drastically. 
- Due to higher rate of hydration at higher temperatures, after $20 \mathrm{~min}$ the irreversible influence of hydration elevates the level of steady state. This accelerates reaching to equilibrium condition and increases the value of $\alpha$. 


\section{CHAPTER 6}

\section{CONCLUSIONS AND FUTURE WORK}

\subsection{Conclusions}

In this dissertation, influence of water content, diatomaceous earth (DE) replacement level, pressure and temperature on fresh and rheological properties of cement pastes, specifically their thixotropic behavior, were investigated. For that purpose, first, fresh properties such as viscosity, yield stress, setting time, bleeding, flowability and heat signature of cement pastes with $\mathrm{w} / \mathrm{b}$ ratios of $0.4,0.5$ and 0.6 and diatomaceous earth replacement levels of $0,2,6$ and $10 \%$ were studied. Then, four simple thixotropic protocols capable of quantitatively measuring two different aspects of thixotropy, i.e. structural breakdown and buildup, were suggested. Then, a series of protocols were proposed to quantitatively measure thixotropic behavior of cementitious pastes. These protocols are intended to give a more comprehensive and reliable understanding of thixotropic behavior of cementitious materials. For that purpose, four different thixotropic protocols were proposed. Two protocols were intended to address the breakdown aspect of thixotropy and the next two protocols its buildup. Theses protocols were then applied on cement pastes with different water contents and DE replacement levels and at different pressures and temperatures. The quantitative results from these protocols were used to analyze breakdown and buildup, separately; and the correlation between these two aspects of thixotropy. Finally, the most common thixotropic model used for cementitious materials and a simplified version of that 
proposed by Roussel were described. Then, the accuracy of the model was investigated by fitting it to the actual results obtained by a rheometer. A test procedure was determined and changes in thixotropic behavior of cement pastes were monitored at various resting times. Cement pastes with different mix proportion and at different temperatures were investigated. Lastly, a comparison between cement pastes flocculation process at rest from obtained data and model's predictions were conducted and some modifications were proposed. Based on these studies, the conclusions with regards to the main objectives of this research can be given as follows:

\subsubsection{Influence of DE on fresh properties of cement pastes}

Findings of this paper show that using Diatomaceous Earth (DE) as a partial replacement for cement has significant influence on fresh and thixotropic properties of cement pastes. The porous shape and high Blaine surface of DE particles makes this natural pozzolanic additive of cement pastes to be highly water absorbent. DE can impact fresh properties of cement pastes as follow:

- Increase in DE content of cement pastes increased the apparent viscosity of cement pastes with $w / b$ ratios of $0.4,0.5$ and 0.6 . It can be due to higher solid volume fraction of cement pastes with higher DE content which leads to higher inter-particle interactions.

- Increase in DE content changed the shear behavior of cement pastes from shear thinning to shear thickening behavior. This change in shear behavior is clearer in cement pastes with 0.5 and $0.6 \mathrm{w} / \mathrm{b}$ ratios.

- Increase in DE content decreased flow diameter of cement pastes at all water to binder ratios. 
- Increase in DE content of cement pastes reduced bleeding rate of cement pastes with $0.4 \mathrm{w} / \mathrm{b}$ ratio.

- Increase in DE content shortened both initial and final setting time of cement pastes at all $\mathrm{w} / \mathrm{b}$ ratios. DE particles improve water diffusion, increase zeta potential and accelerate formation of hydration products which lead to shorter setting times.

- Increase in DE content lowers the hydration heat of cement pastes at all water to binder ratios due to lower cement content of cement pastes.

- Results from isothermal calorimetry show that increase in replacement level of cement with DE hasten the hydration process in cement pastes with all water to cement ratios which is in good agreement with results from setting time test.

\subsubsection{Influence of DE on thixotropic behavior of cement pastes}

- Increase in DE content of cement pastes can decrease the amount of energy required to breakdown cement pastes structures to their most practically possible deflocculated state (steady state) under certain shear rate but does not significantly change minimum shear stress needed to initiate their flow (structure breakdown).

- No clear trend of build-up ratio of cement paste with different DE contents was observed.

- Increase in DE replacement level to some extent, increases solid volume fraction and consequently increases initial state of flocculation and rate of flocculation. However, rate of increase in flocculation evolution decreases by increase in level of DE replacement level. 
- If the level of DE replacement level exceeds an optimum level it will decrease thixotropic properties of cement pastes due to low concentration of cement particles in cement pastes and negatively affects flocculation process.

- For short resting times cement pastes with higher DE content show higher deflocculation rate because of their higher state of flocculation caused by colloidal forces. However, at longer resting times, higher colloidal forces and higher solid volume fraction compare to cement pastes with less DE content results in stronger hydration nucleation bonds and consequently lower de-flocculation rate.

- No clear correlation between breakdown and build-up aspects of different cement pastes was observed. Hence, for each cement paste with specific mix proportion both aspects should be investigated.

\subsubsection{Influence of pressure and temperature on thixotropic behavior of cement pastes}

- Increase in temperature results in acceleration in hydration and flocculation rate of cement pastes, which consequently leads to higher static yield stress and build-up ratio.

- Impact of high pressure up to $20 \mathrm{MPa}$ on thixotropic behavior of cement pastes with $0.6 \mathrm{w} / \mathrm{b}$ ratio is minimal and can be neglected.

- Up to 20 min resting time, slight increase in state of flocculation is observed by increase in temperature. After $20 \mathrm{~min}$, state of flocculation increases significantly with increase in temperature.

- increase in temperature of cement paste gradually changes its structural buildup pattern at rest, from a convergence trend to a concave trend with increasing slopes. 
This change is especially more obvious at temperatures higher than $25^{\circ} \mathrm{C}$ and resting times higher than $20 \mathrm{~min}$.

- Due to higher rate of hydration at higher temperatures, after $20 \mathrm{~min}$ the irreversible influence of hydration elevates the level of steady state. This accelerates reaching to equilibrium condition and increases the value of $\alpha$.

\subsubsection{Thixotropic model}

In general, the simplified thixotropic model proposed by Roussel is a reliable and practical platform to predict and monitor thixotropic behavior of cementitious materials. However, the following points should be considered in its application:

- The simplified model seems to be more accurate for concrete and mortar but in case of cement paste some modifications based on mix proportion may be needed.

- Unlike what model assumes, the flocculation process of cement paste is not linear, especially at short resting times.

- Increase in temperature of cement paste gradually changes its structural buildup pattern at rest, from a convergence trend to a concave trend with increasing slopes. This change is especially more obvious at temperatures higher than $25^{\circ} \mathrm{C}$ and resting times higher than $20 \mathrm{~min}$.

- Up to 20 min resting time, changes in de-flocculation process is less sensitive to changes in temperatures. But for longer resting times, $\alpha$ increases drastically.

- Due to higher rate of hydration at higher temperatures, after $20 \mathrm{~min}$ the irreversible influence of hydration elevates the level of steady state. This accelerates reaching to equilibrium condition and increases the value of $\alpha$. 
- Modifications should be considered in applying model to cementitious materials with temperatures higher than $25^{\circ} \mathrm{C}$.

\subsection{Future works}

Due to importance of thixotropic behavior of cementitious materials on their performance, this work suggests future study on this area. Some future work topics that can be investigate are as follows:

- This work studied the influence of DE as a partial replacement for cement on thixotropic behavior of cement pastes. No chemical admixtures were used. The study can be extended to a cementitious system with both mineral and chemical admixtures used to further investigate the compatibility among different ingredients.

- The focus of this study was solely on thixotropic behavior of cement pastes. How the addition of fine and coarse aggregates influences these behaviors and the correlation between thixotropic behaviors of cement paste, mortar and concrete can be investigated.

- This work shows that increase in temperature of cement paste gradually changes its structural buildup pattern at rest, from a convergence trend to a concave trend with increasing slopes. This change is especially more obvious at temperatures higher than $25{ }^{\circ} \mathrm{C}$ and resting times longer than $20 \mathrm{~min}$. More focused study on these behavioral changes at high temperatures and how to modify thixotropic models to better represent influence of temperature can be conducted. 
- This work studied thixotropic behavior of cement pastes on macroscopic scale. To fully understand why these behaviors change the way they do, a microscopic study on their structure and the correlation between macroscopic and microscopic aspects of thixotropy seems to be vital. 


\section{REFERENCES}

Agullo, L., Toralles-Carbonari, B., Gettu, R., \& Aguado, A. (1999). Fluidity of cement pastes with mineral admixtures and superplasticizer — a study based on the Marsh cone test. Materials and Structures, 32(7), 479-485.

Assaad, J., \& Khayat, K. H. (2004). Assessment of thixotropy of self-consolidating concrete and concrete-equivalent-mortar-effect of binder composition and content. Materials Journal, 101(5), 400-408.

Assaad, J., Khayat, K. H., \& Mesbah, H. (2003). Assessment of thixotropy of flowable and self-consolidating concrete. Materials Journal, 100(2), 99-107.

ASTM. (2008). Standard Test Method for Time of Setting of Hydraulic Cement by Vicat Needle: ASTM International West Conshohocken, Pa.

ASTM. (2009). Standard test method for measurement of heat of hydration of hydraulic cementitious materials using isothermal conduction calorimetry: ASTM International West Conshohocken, Pa.

Aydin, A. C., \& Gül, R. (2007). Influence of volcanic originated natural materials as additives on the setting time and some mechanical properties of concrete. Construction and Building Materials, 21(6), 1277-1281.

Bakr, H. (2010). Diatomite: Its characterization, modifications and applications. Asian journal of materials science, 2(3), 121-136.

Banfill, P. (1990). Rheology of Fresh Cement and Concrete: Proceedings of an International Conference, Liverpool, 1990: CRC Press. 
Banfill, P. (2003). THE RHEOLOGY OF FRESH CEMENT AND CONCRETE - A RE-

VIEW. Paper presented at the International Cement Chemistry Congress, Durban.

Banfill, P. (2006). Rheology of fresh cement and concrete. Rheology reviews, 2006, 61.

Barnes, H. A. (1997). Thixotropy—a review. Journal of Non-Newtonian fluid mechanics, $70(1-2), 1-33$.

Bouras, R., Kaci, A., \& Chaouche, M. (2012). Influence of viscosity modifying admixtures on the rheological behavior of cement and mortar pastes. Korea-Australia Rheology Journal, 24(1), 35-44.

Cheng, D. C., \& Evans, F. (1965). Phenomenological characterization of the rheological behaviour of inelastic reversible thixotropic and antithixotropic fluids. British Journal of Applied Physics, 16(11), 1599.

Coussot, P., Nguyen, Q. D., Huynh, H., \& Bonn, D. (2002). Avalanche behavior in yield stress fluids. Physical review letters, 88(17), 175501.

Degirmenci, N., \& Yilmaz, A. (2009). Use of diatomite as partial replacement for Portland cement in cement mortars. Construction and Building Materials, 23(1), 284-288.

Dolley, P. (1999). Diatomite: Minerals Yearbook: Volume I-Metals and Minerals. US Geological Survey, 1-4.

Ergün, A. (2011). Effects of the usage of diatomite and waste marble powder as partial replacement of cement on the mechanical properties of concrete. Construction and Building Materials, 25(2), 806-812.

Ferraris, C. F., Obla, K. H., \& Hill, R. (2001). The influence of mineral admixtures on the rheology of cement paste and concrete. Cement and concrete research, 31(2), 245255. 
Ferron, R. P., Gregori, A., Sun, Z., \& Shah, S. P. (2007). Rheological method to evaluate structural buildup in self-consolidating concrete cement pastes. ACI materials journal, 104(3), 242.

Ferron, R. P. D. (2008). Formwork pressure of self-consolidating concrete: influence of flocculation mechanisms, structural rebuilding, thixotropy and rheology. Northwestern University.

Fragoulis, D., Stamatakis, M. G., Papageorgiou, D., \& Chaniotakis, E. (2005). The physical and mechanical properties of composite cements manufactured with calcareous and clayey Greek diatomite mixtures. Cement and Concrete Composites, 27(2), 205209.

He, Q., Gong, X., Xuan, S., Jiang, W., \& Chen, Q. (2015). Shear thickening of suspensions of porous silica nanoparticles. Journal of Materials Science, 50(18), 6041-6049.

Jarny, S., Roussel, N., Rodts, S., Bertrand, F., Le Roy, R., \& Coussot, P. (2005). Rheological behavior of cement pastes from MRI velocimetry. Cement and concrete research, 35(10), 1873-1881.

Jud Sierra, E., Miller, S. A., Sakulich, A. R., MacKenzie, K., \& Barsoum, M. W. (2010). Pozzolanic activity of diatomaceous earth. Journal of the American Ceramic Society, 93(10), 3406-3410.

Kastis, D., Kakali, G., Tsivilis, S., \& Stamatakis, M. G. (2006). Properties and hydration of blended cements with calcareous diatomite. Cement and concrete research, $36(10), 1821-1826$.

Kim, J. H., Kwon, S. H., Kawashima, S., \& Yim, H. J. (2017). Rheology of cement paste under high pressure. Cement and Concrete Composites, 77, 60-67. 
Liu, F., Sun, Z., \& Qi, C. (2014). Raman spectroscopy study on the hydration behaviors of Portland cement pastes during setting. Journal of Materials in Civil Engineering, $27(8), 04014223$.

Lootens, D., Hébraud, P., Lécolier, E., \& Van Damme, H. (2004). Gelation, shear-thinning and shear-thickening in cement slurries. Oil \& gas science and technology, 59(1), $31-40$.

Mejía, J. M., de Gutiérrez, R. M., \& Montes, C. (2016). Rice husk ash and spent diatomaceous earth as a source of silica to fabricate a geopolymeric binary binder. Journal of Cleaner Production, 118, 133-139.

Mewis, J., \& Wagner, N. J. (2009). Thixotropy. Advances in Colloid and Interface Science, 147, 214-227.

Miller, S. A., Sakulich, A. R., Barsoum, M. W., \& Jud Sierra, E. (2010). Diatomaceous Earth as a Pozzolan in the Fabrication of an Alkali-Activated Fine-Aggregate Limestone Concrete. Journal of the American Ceramic Society, 93(9), 2828-2836.

Mostafa, N. Y., \& Brown, P. W. (2005). Heat of hydration of high reactive pozzolans in blended cements: isothermal conduction calorimetry. Thermochimica acta, 435(2), $162-167$.

Nägele, E. (1985). The zeta-potential of cement. Cement and concrete research, 15(3), 453-462.

NRMCA. (2016). Ready Mixed Concrete Production Statistics. Retrieved from https://www.nrmca.org/concrete/data.asp 
Ouyang, J., Tan, Y., Corr, D. J., \& Shah, S. P. (2016). The thixotropic behavior of fresh cement asphalt emulsion paste. Construction and Building Materials, 114, 906912.

Ovarlez, G., \& Roussel, N. (2006). A physical model for the prediction of lateral stress exerted by self-compacting concrete on formwork. Materials and Structures, 39(2), 269-279.

Pang, X., Bentz, D. P., Meyer, C., Funkhouser, G. P., \& Darbe, R. (2013). A comparison study of Portland cement hydration kinetics as measured by chemical shrinkage and isothermal calorimetry. Cement and Concrete Composites, 39, 23-32.

Plank, J., \& Hirsch, C. (2007). Impact of zeta potential of early cement hydration phases on superplasticizer adsorption. Cement and concrete research, 37(4), 537-542.

Qian, Y., \& Kawashima, S. (2016). Flow onset of fresh mortars in rheometers: Contribution of paste deflocculation and sand particle migration. Cement and concrete research, 90, 97-103.

Quanji, Z. (2010). Thixotropic behavior of cement-based materials: effect of clay and cement types.

Rahhal, V., \& Talero, R. (2009). Calorimetry of Portland cement with silica fume, diatomite and quartz additions. Construction and Building Materials, 23(11), 33673374.

Robert D. Crangle, J. (2013). DIATOMITE, Mineral Yearbook 2013. from U.S. Geological Survey

Roussel, N. (2006). A thixotropy model for fresh fluid concretes: theory, validation and applications. Cement and concrete research, 36(10), 1797-1806. 
Roussel, N., Ovarlez, G., Garrault, S., \& Brumaud, C. (2012). The origins of thixotropy of fresh cement pastes. Cement and concrete research, 42(1), 148-157.

Sally Jewell, \& Kimball, S. M. (2015). MINERAL COMMODITY

SUMMARIES 2015. from U.S. Geological Survey

Sally Jewell, \& Kimball, S. M. (2016). MINERAL COMMODITY

SUMMARIES 2016. from U.S. Geological Survey

Stamatakis, M. G., Fragoulis, D., Csirik, G., Bedelean, I., \& Pedersen, S. (2003). The influence of biogenic micro-silica-rich rocks on the properties of blended cements. Cement and Concrete Composites, 25(2), 177-184.

Sun, Z., \& Young, C. (2014). Bleeding of SCC pastes with fly ash and GGBFS replacement. Journal of Sustainable Cement-Based Materials, 3(3-4), 220-229.

Taylor, H. F. (1997). Cement chemistry: Thomas Telford.

Wallevik, J. E. (2006). Relationship between the Bingham parameters and slump. Cement and concrete research, 36(7), 1214-1221.

Wallevik, J. E. (2009). Rheological properties of cement paste: thixotropic behavior and structural breakdown. Cement and concrete research, 39(1), 14-29.

Yahia, A. (2011). Shear-thickening behavior of high-performance cement grouts-Influencing mix-design parameters. Cement and concrete research, 41(3), 230-235.

Yahia, A. (2014). Effect of solid concentration and shear rate on shear-thickening response of high-performance cement suspensions. Construction and Building Materials, 53, 517-521.

Y1lmaz, B., \& Ediz, N. (2008). The use of raw and calcined diatomite in cement production. Cement and Concrete Composites, 30(3), 202-211. 


\title{
CURRICULUM VITA
}

\author{
Bashir Hasanzadeh
}

Address: 3201 Leith Lane Apt\#513, Louisville, KY 40218

Mobile: (502)-807-6601

Email: bashir.hasanzadeh@gmail.com, bashir.hasanzadeh@1ouisville.edu

\section{Education}

Expected Dec. 2017 PhD in Civil Engineering - (GPA: 3.92/4.00)

University of Louisville, Louisville, KY, US

Courses: Construction Materials, Material Characterizations, Non-Destructive Testing (NDT) Methods, Advanced Mechanic of Solids, Pavement Design, Traffic Engineering, Intelligent Transportation Systems (ITS), Earth Pressure \& Retaining Structures

MSc in Construction Management - (graduated with Merit)

School of Civil Engineering, University of Birmingham, UK

Courses: Construction Management, Construction Technology, Engineering Production and Risk Management in Construction, Sustainable Construction, Group Working in the Construction Industry, Finance and Core Skills

2010

\section{BSc in Civil Engineering}

Ferdowsi University of Mashhad (FUM), IR

\section{Research and Teaching Experience}

$>$ Lab Instructor - "Advanced Material Analytic Research" and "Concrete" Labs, University of Louisville, Louisville, KY 2015-present

- Training and instructing undergraduate and graduate students on how to use lab's equipment and conduct standard testing methods.

$>$ Teaching Assistant - University of Louisville 2014-2015

Courses: Concrete Design, Steel Design, Mechanics of Material Lab, Indeterminate Structural Analysis, Structural analysis

$>$ Research Assistant - University of Louisville, Louisville, KY, US 2015-2017 Highlight of projects:

- "Improve the stability of Oilwell Cementing via Rheological Methods" Funded by Kentucky Science and Engineering Foundation 
- "Acquisition of a Pressure Rheometer with Structural Analysis Accessories for Oil Well Cement and Other Materials Characterization" Funded by the National Science Foundation

$\circ$ investigated thixotropic behavior of different cement pastes under high pressure and various temperatures

- "Characterizing Paste Microstructure during Setting with Combined Raman Spectroscopy and Confocal Microscopy" Funded by the National Science Foundation

- Studying influence of Carbon Nanotubes on flowability of self-consolidating concrete (SCC)

- Investigating effect of high temperature and pressure on organo-montmorillonite in oil drilling based fluids

Research Assistant - University of Birmingham, Birmingham, UK 2010-2011

- Applying process of Risk Management on construction of a supposed bridge project

- Feasibility study on renewable energy sources for a demonstration development at "Coed Darcy" a site in the south Wales

- Study on main sources of noise emitted from wind turbines, effective parameters on noise perception/annoyance by nearby residents and probable cause and source of Aerodynamic Modulation

\section{Technical/Computer Skills}

- Software: AutoCAD, Primavera (P6), Microsoft Project, Bluebeam, LEAP Bridge Concrete/Steel, ETABS, SAFE, RAM Elements.

- Applications: Microsoft Office Suite (Word, Excel, PowerPoint, etc.)

- Operating Systems: Microsoft Windows, Mac OS X, iOS

- Laboratory techniques: Rheology, Raman Spectroscopy, XRD, SEM, Calorimetry, TGA

\section{Certificate and Professional Membership}

- Membership: American Concrete Institute (ACI), American Society of Civil Engineers (ASCE), American Standard Testing Methods (ASTM) international

- Certificate: ACI Concrete Field Testing Technician - grade I.

\section{Honors/Awards}

- Awarded graduate research assistant, University of Louisville, Fall 2015-present

- Awarded graduate teaching assistant, University of Louisville, Spring 2014, Fall 2014, Spring 2015, Summer 2015

- Awarded graduate travel fund, Kentucky Science and Engineering Foundation (KSEF), Fall 2016

- Awarded graduation with merit, University of Birmingham, Fall 2011

- Ranked in the top 10\% students of the Civil Engineering department, Ferdowsi University of Mashhad (FUM) Spring 2010

\section{Publication}

- Hasanzadeh, Bashir, Fengjuan Liu, and Zhihui Sun. "Monitoring hydration of UHPC and conventional paste by quantitative analysis on Raman patterns." Construction and Building Materials 114 (2016): 208-214.

- Mahyar Ramezani, Young Hoon Kim, Bashir Hasanzadeh, Zhihui Sun. "Influence of Carbon Nanotubes on SCC Flowability.” Accepted to SCC 2016, 8th International RILEM Symposium on Self-Compacting Concrete, May 16, 2016. 
- Hasanzadeh, Bashir, and Zhihui Sun. "Impacts of Diatomaceous Earth as Partial Replacement for Cement on Fresh Properties of Cement Pastes". Submitted to Journal of Construction and Building Materials. 